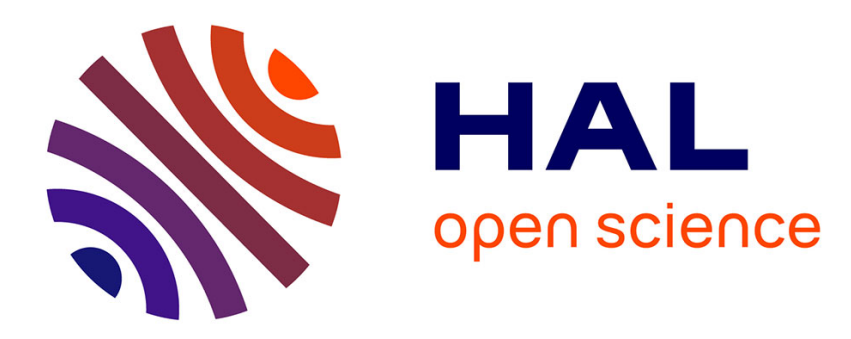

\title{
On a conjecture by Pierre Cartier about a group of associators.
}

Vincel Hoang Ngoc Minh

\section{To cite this version:}

Vincel Hoang Ngoc Minh. On a conjecture by Pierre Cartier about a group of associators.. 2011. hal-00423455v11

\section{HAL Id: hal-00423455 \\ https://hal.science/hal-00423455v11}

Preprint submitted on 9 Jun 2012

HAL is a multi-disciplinary open access archive for the deposit and dissemination of scientific research documents, whether they are published or not. The documents may come from teaching and research institutions in France or abroad, or from public or private research centers.
L'archive ouverte pluridisciplinaire HAL, est destinée au dépôt et à la diffusion de documents scientifiques de niveau recherche, publiés ou non, émanant des établissements d'enseignement et de recherche français ou étrangers, des laboratoires publics ou privés. 


\title{
On a conjecture by Pierre Cartier about a group of associators
}

\section{Hoang Ngoc Minh}

LIPN - UMR 7030, CNRS, 93430 Villetaneuse, France.

Université Lille II, 1, Place Déliot, 59024 Lille, France.

\begin{abstract}
In [8], Pierre Cartier conjectured that for any non commutative formal power series $\Phi$ on $X=\left\{x_{0}, x_{1}\right\}$ with coefficients in a $\mathbb{Q}$-extension, $A$, subjected to some suitable conditions, there exists an unique algebra homomorphism $\varphi$ from the $\mathbb{Q}$-algebra generated by the convergent polyzêtas to $A$ such that $\Phi$ is computed from $\Phi_{K Z}$ Drinfel'd associator by applying $\varphi$ to each coefficient. We prove $\varphi$ exists and it is a free Lie exponential over $X$. Moreover, we give a complete description of the kernel of polyzêta and draw some consequences about a structure of the algebra of convergent polyzêtas and about the arithmetical nature of the Euler constant.
\end{abstract}

\section{Contents}

1 Introduction 2

1.1 Drinfel'd associator and polyzêtas . . . . . . . . . . . . . 2

1.2 Group of associators and regularized Chen generating series . . . 4

1.3 Global renormalization and global regularization . . . . . . . 4

2 Background : structures and analytical studies of harmonic sums and of polylogarithms

2.1 Structures of harmonic sums and of polylogarithms

2.1.1 Quasi-symmetric functions and harmonic sums . . . . . 6

2.1.2 Iterated integrals and polylogarithms . . . . . . . . . . 7

2.2 Results à la Abel for generating series of harmonic sums and of polylogarithms . . . . . . . . . . . . 8

2.2.1 Generating series of harmonic sums and of polylogarithms 8

2.2.2 Asymptotic expansions by noncommutative generating se-

ries and regularized Chen generating series . . . . . . . 11

2.3 Indiscernability over a class of formal power series . . . . . . . 14

2.3.1 Residual calculus and representative series . . . . . . . . 14

2.3.2 Continuity and indiscernability . . . . . . . . . 15

3 Group of associators : polynomial relations among convergent polyzêtas and identification of local coordinates $\quad 17$

3.1 Generalized Euler constants and global regularization of polyzêtas 17 3.1.1 Three regularizations of divergent polyzêtas . . . . . . . . 17

3.1.2 Identities of noncommutative generating series of polyzêtas 20

3.2 Action of differential Galois group of polylogarithms on their asymptotic expansions . . . . . . . . . . . . . . . 21 
3.2.1 Group of associators theorem . . . . . . . . . . . . . 21

3.2.2 Concatenation of Chen generating series . . . . . . . . 25

3.3 Algebraic combinatorial studies of polynomial relation among polyzêta via a group of associators . . . . . . . . . . . . . 26

3.3.1 Preliminary study . . . . . . . . . . . . . . 26

3.3.2 Description of polynomial relations among coefficients of associator and irreducible polyzêtas . . . . . . . . . 27

4 Concluding remarks : complete description of $\operatorname{ker} \zeta$ and structure of polyzêtas

4.1 A conjecture by Pierre Cartier . . . . . . . . . . . . . . . 33

4.2 Arithmetical nature of $\gamma \ldots \ldots \ldots \ldots \ldots \ldots$

4.3 Structure and arithmetical nature of polyzêtas . . . . . . . 35

5 Annexe A : pair of bases in duality and proof of Theorem $2 \quad 36$

5.1 Preliminary results . . . . . . . . . . . . . 36

5.2 Pair of bases in duality . . . . . . . . . . . . . . . . . . . 39

5.3 Proof of Theorem 2 . . . . . . . . . . . . . . . . 46

6 Annexe B : differential realization 446

6.1 Polysystem and convergence criterion . . . . . . . . . 46

6.1.1 Serial estimates from above . . . . . . . . . . . . . 46

6.1.2 Upper bounds à la Cauchy . . . . . . . . . . . . . . . 49

6.2 Polysystems and nonlinear differential equation . . . . . . . . 51

6.2.1 Nonlinear differential equation (with three singularities) . 51

6.2.2 Asymptotic behaviour of the successive differentiation of

the output via extended Fliess fundamental formula . . . 52

6.3 Differential realization . . . . . . . . . . . . . . 53

6.3.1 Differential realization . . . . . . . . . . . . . 53

6.3.2 Fliess' local realization theorem . . . . . . . . . . . . . 55

\section{Introduction}

\subsection{Drinfel'd associator and polyzêtas}

In 1986, in order to study the linear representation of the braid group $B_{n}$ coming from the monodromy of the Knizhnik-Zamolodchikov differential equations over $\mathbb{C}_{*}^{n}=\left\{\underline{z}=\left(z_{1}, \ldots, z_{n}\right) \in \mathbb{C}^{n} \mid z_{i} \neq z_{j}\right.$ for $\left.i \neq j\right\}[14]:$

$$
d F(\underline{z})=\Omega_{n}(\underline{z}) F(\underline{z}) \quad \text { with } \quad \Omega_{n}(\underline{z})=\frac{1}{2 \mathrm{i} \pi} \sum_{1 \leq i<j \leq n} t_{i, j} \frac{d\left(z_{i}-z_{j}\right)}{z_{i}-z_{j}}
$$

and $\left\{t_{i, j}\right\}_{i, j \geq 1}$ are noncommutative variables, Drinfel'd introduced a class of formal power series $\Phi$ on noncommutative variables over the finite alphabet $X=\left\{x_{0}, x_{1}\right\}$. Such a power series $\Phi$ is called an associator. 
Since the system (1) is completely integrable then $d \Omega_{n}-\Omega_{n} \wedge \Omega_{n}=0[7,14]$. It is equivalent to the fact the $\left\{t_{i, j}\right\}_{i, j \geq 1}$ satisfy the infinitesimal braid relations :

$$
\begin{aligned}
& t_{i, j}=0 \quad \text { for } \quad i=j, \\
& t_{i, j}=t_{j, i} \quad \text { for } \quad i \neq j, \\
& {\left[t_{i, j}, t_{i, k}+t_{j, k}\right]=0 \quad \text { for distinct } \quad i, j, k \text {, }} \\
& {\left[t_{i, j}, t_{k, l}\right]=0 \quad \text { for distinct } \quad i, j, k, l \text {. }}
\end{aligned}
$$

Example 1. - $\mathcal{T}_{2}=\left\{t_{1,2}\right\}$.

$$
\Omega_{2}\left(z_{1}, z_{2}\right)=\frac{t_{1,2}}{2 \mathrm{i} \pi} \frac{d\left(z_{1}-z_{2}\right)}{z_{1}-z_{2}} \quad \text { with } \quad F\left(z_{1}, z_{2}\right)=\left(z_{1}-z_{2}\right)^{t_{1,2} / 2 \mathrm{i} \pi} .
$$

- $\mathcal{T}_{3}=\left\{t_{1,2}, t_{1,3}, t_{2,3}\right\},\left[t_{1,3}, t_{1,2}+t_{2,3}\right]=\left[t_{2,3}, t_{1,2}+t_{1,3}\right]=0$.

$$
\begin{aligned}
\Omega_{3}\left(z_{1}, z_{2}, z_{3}\right) & =\frac{1}{2 \mathrm{i} \pi}\left[t_{1,2} \frac{d\left(z_{1}-z_{2}\right)}{z_{1}-z_{2}}+t_{1,3} \frac{d\left(z_{1}-z_{3}\right)}{z_{1}-z_{3}}+t_{2,3} \frac{d\left(z_{2}-z_{3}\right)}{z_{2}-z_{3}}\right] . \\
F\left(z_{1}, z_{2}, z_{3}\right) & =G\left(\frac{z_{1}-z_{2}}{z_{1}-z_{3}}\right)\left(z_{1}-z_{3}\right)^{\left(t_{1,2}+t_{1,3}+t_{2,3}\right) / 2 \mathrm{i} \pi}
\end{aligned}
$$

where $G$ satisfies the following fuchsian differential equation with three regular singularities at 0,1 and $\infty$ :

$$
d G(z)=\left[x_{0} \omega_{0}(z)+x_{1} \omega_{1}(z)\right] G(z),
$$

with

$$
\begin{aligned}
& x_{0}:=\frac{t_{1,2}}{2 \mathrm{i} \pi} \quad \text { and } \quad \omega_{0}(z):=\frac{d z}{z}, \\
& x_{1}:=-\frac{t_{2,3}}{2 \mathrm{i} \pi} \quad \text { and } \quad \omega_{1}(z):=\frac{d z}{1-z} .
\end{aligned}
$$

As already shown by Drinfel'd, the equation $(D E)$ admits, on the simply connected domain $\mathbb{C}-(]-\infty, 0] \cup[1,+\infty[)$, two specific solutions

$$
G_{0}(z) \underset{z \rightsquigarrow 0}{\widetilde{2}} \exp \left[x_{0} \log (z)\right] \quad \text { and } \quad G_{1}(z) \underset{z \rightsquigarrow 1}{\widetilde{e x p}}\left[-x_{1} \log (1-z)\right] .
$$

He also proved there exists the associator $\Phi_{K Z}$ such that $G_{1}^{-1}(z) G_{0}(z)=\Phi_{K Z}$.

After that, Lê and Murakami expressed the coefficients of the Drinfel'd associator $\Phi_{K Z}$ in terms of convergent polyzêtas [22], i.e. for $r_{1}>1$,

$$
\zeta\left(r_{1}, \ldots, r_{k}\right)=\sum_{n_{1}>\ldots>n_{k}>0} \frac{1}{n_{1}^{r_{1}} \ldots n_{k}^{r_{k}}} .
$$

In [22], the authors also expressed the divergent coefficients as linear combinations of convergent polyzêtas via a regularization process (see also [38]). This process is one of many ways to regularize the divergent terms. 


\subsection{Group of associators and regularized Chen generating series}

The algebraic aspects of our regularization process based essentially on various products $^{1}$ among polyzêtas (see [33]) and its analytical aspects will be described, in Section 3.1, as the finite part, of the asymptotic expansions in different scales of comparison ${ }^{2}$ [5]. It will be seen also, in Section 3.2, as the action of the differential Galois group of the polylogarithms ${ }^{3}$ (recalled in Section 2.1.2)

$$
\operatorname{Li}_{r_{1}, \ldots, r_{k}}(z)=\sum_{n_{1}>\ldots>n_{k}>0} \frac{z^{n_{1}}}{n_{1}^{r_{1}} \ldots n_{k}^{r_{k}}}
$$

on the asymptotic expansion of polylogarithms, at $z=1$ and in the comparison scale $\left\{(1-z)^{a} \log ^{b}(1-z)\right\}_{a \in \mathbb{Z}, b \in \mathbb{N}}$, and the same action on the asymptotic expansions, at $+\infty$ and in the comparison scales $\left\{n^{a} \log ^{b}(n)\right\}_{a \in \mathbb{Z}, b \in \mathbb{N}}$ and $\left\{n^{a} \mathrm{H}_{1}^{b}(n)\right\}_{a \in \mathbb{Z}, b \in \mathbb{N}}$, of the harmonic sums (recalled in Section 2.1.1)

$$
\mathrm{H}_{r_{1}, \ldots, r_{k}}(N)=\sum_{n_{1}>\ldots>n_{k}>0}^{N} \frac{1}{n_{1}^{r_{1}} \ldots n_{k}^{r_{k}}} .
$$

This action leads then to a conjecture by Pierre Cartier ([8], conjecture C3) and to the description of the group of associators yielding the ideal of polynomial relations among coefficients of associators (theorems 13 and 14). This group is in fact, closely linked to the group of the Chen generating series studied by K.T. Chen to describe the solutions of differential equations [10] and it turns out that each associator regularizes a Chen generating series of the differential forms $\omega_{0}$ and $\omega_{1}$ along the integration path on the simply connected domain $\mathbb{C}-(]-\infty, 0] \cup[1,+\infty[)$.

\subsection{Global renormalization and global regularization}

In fact, our regularization process based essentially on two noncommutative generating series over $Y=\left\{y_{i}\right\}_{i \geq 1}$, which encodes the multi-indices $\left(r_{1}, \ldots, r_{k}\right)$ by the words $y_{r_{1}} \ldots y_{r_{k}}$ over the monoid generated by $Y$, denoted by $Y^{*}$, of polylogarithms and of harmonic sums (recalled in Section 2.2.1)

$$
\Lambda(z)=\sum_{w \in Y^{*}} \operatorname{Li}_{w}(z) w \text { and } \mathrm{H}(N)=\sum_{w \in Y^{*}} \mathrm{H}_{w}(N) w
$$

Through the algebraic combinatorial aspects ${ }^{4}$ [45] and the topological aspects [2] of formal power series in noncommutative variables, we have already

\footnotetext{
${ }^{1}$ First source of ambiguity leading to the problem of rewriting expressions of polyzêtas in a canonical form using irreducible Lyndon words (see [34, 32]).

${ }^{2}$ Second source of ambiguity leading to the problem to determine the value of regularized polyzêtas and its analytical meaning (see [33, 37]).

${ }^{3}$ Third source of ambiguity leading to the problem of fixing the integration path to solve $(D E)$ and its monodromy group (see [35]) or its differential Galois group (see [38])

${ }^{4}$ See [45] to get an idea of these aspects of combinatorial Hopf algebra of the shuffle product,
} 
showed the existence of noncommutative formal series over $Y, Z_{1}$ and $Z_{2}$ with constant terms, such that [39]

$$
\begin{aligned}
\lim _{z \rightarrow 1} \exp \left(y_{1} \log \frac{1}{1-z}\right) \Lambda(z) & =Z_{1}, \\
\lim _{N \rightarrow \infty} \exp \left(\sum_{k \geq 1} \mathrm{H}_{y_{k}}(N) \frac{\left(-y_{1}\right)^{k}}{k}\right) \mathrm{H}(N) & =Z_{2} .
\end{aligned}
$$

Moreover, $Z_{1}$ and $Z_{2}$ are equal and stand for the noncommutative generating series of all convergent polyzêtas $\{\zeta(w)\}_{w \in Y^{*}-y_{1} Y^{*}}$ as shown by the factorized form indexed by Lyndon words (recalled in Section 2.2). This theorem enables, in particular, to explicit the counter-terms eliminating the divergence of the polylogarithms $\left\{\operatorname{Li}_{w}(z)\right\}_{w \in y_{1} Y^{*}}$, for $z \rightarrow 1$, and of the harmonic sums $\left\{\mathrm{H}_{w}(N)\right\}_{w \in y_{1} Y^{*}}$, for $N \rightarrow \infty$, and to calculate the Euler-Mac Laurin constants associated to the divergent polyzêtas $\{\zeta(w)\}_{w \in y_{1} Y^{*}}$ (see Corollary 4 ). It allows also to give, in Section 3.3 and via identification of locale coordinates in infinite dimension, a complete description of the kernel by its generators, of the following algebra homomorphism ${ }^{5}$

$$
\begin{aligned}
\zeta:\left(A \epsilon \oplus\left(Y-y_{1}\right) A\langle Y\rangle, \pm\right) & \longrightarrow(\mathbb{R}, .) \\
y_{r_{1}} \ldots y_{r_{k}} & \longmapsto \sum_{n_{1}>\ldots>n_{k}>0} \frac{1}{n_{1}^{r_{1}} \ldots n_{k}^{r_{k}}},
\end{aligned}
$$

and the set of $A$-irreducible polyzêtas forming a transcendence basis of the image of $\zeta$, with $A=\mathbb{Q}[\mathrm{i} \pi]$ (see Corollary 10).

Finally, via the indiscernability (recalled in Section 2.3) over the group of associators, this study makes precise the structure of the $A$-algebra generated by the convergent polyzêtas (see Theorem 19) and concludes the main challenge of the polynomial relations among polyzêtas indexed by convergent Lyndon words which are algebraicly independant on the Euler constant and motivated $[32,34$, $3,49]$. In particular, the $A$-algebra generated by the convergent polyzêtas was conjectured to be free $[32,34]$ and it will be proved, thanks to the propositions 15,16 and 17. Moreover, this free $A$-algebra is graded by weight meaning there is no linear relation among convergent polyzêtas of different weight (see Theorem $19)$.

denoted by $\sqcup$, and its co-product, denoted by $\Delta_{\uplus}$. For the quasi-shuffle product, denoted by $\mathbf{\leftarrow}$, and its co-product, denoted by $\Delta \mathbf{t}$, see Annexe A.

In our works, recalled in Annexe B, these algebraic combinatorial aspects were explored systematically to expand the outputs of nonlinear controlled dynamical system with singular inputs (Corollary 17) on polylogarithmic functional basis [26, 27, 31]. In this way [39], polyzêtas do appear then as fundamental arithmetical constant for the asymptotic analysis and for the renormalization of the outputs and their successive derivations (Corollary 18) via the extended Fliess fundamental formula (Theorem 23).

${ }^{5}$ Here, $\epsilon$ stands for the empty word over $Y$. 


\section{Background : structures and analytical stud- ies of harmonic sums and of polylogarithms}

\subsection{Structures of harmonic sums and of polylogarithms}

\subsubsection{Quasi-symmetric functions and harmonic sums}

Let $\left\{t_{i}\right\}_{i \in \mathbb{N}_{+}}$be an infinite set of variables. The elementary symmetric functions $\eta_{k}$ and the power sums $\psi_{k}$ are defined by (see [45])

$$
\eta_{k}(\underline{t})=\sum_{n_{1}>\ldots>n_{k}>0} t_{n_{1}} \ldots t_{n_{k}} \text { and } \quad \psi_{k}(\underline{t})=\sum_{n>0} t_{n}^{k} .
$$

They are respectively coefficients of the following generating functions

$$
\eta(\underline{t} \mid z)=\prod_{i \geq 1}\left(1+t_{i} z\right) \quad \text { and } \quad \psi(\underline{t} \mid z)=\sum_{i \geq 1} \frac{t_{i} z}{1-t_{i} z}
$$

These generating functions satisfy a Newton identity

$$
z \frac{d}{d z} \log \eta(\underline{t} \mid z)=\psi(\underline{t} \mid-z) .
$$

The fundamental theorem from symmetric functions theory asserts that $\left\{\eta_{k}\right\}_{k \geq 0}$ are linearly independent, and provides remarkable identities like (with $\eta_{0}=1$ ) :

$$
\eta_{k}=\frac{(-1)^{k}}{k !} \sum_{\substack{s_{1}, \ldots, s_{k} \geq 0 \\
s_{1}+\ldots+k s_{k}=k+1}}\left(\begin{array}{c}
k \\
s_{1}, \ldots, s_{k}
\end{array}\right)\left(-\frac{\psi_{1}}{1}\right)^{s_{1}} \ldots\left(-\frac{\psi_{k}}{k}\right)^{s_{k}}
$$

Let $Y$ be the infinite alphabet $\left\{y_{i}\right\}_{i \geq 1}$ equipped with the order $y_{1}>y_{2}>$ $y_{3}>\ldots$ and let $\mathcal{L} y n Y$ be the set of Lyndon words over $Y$. The length of $w=y_{s_{1}} \ldots y_{s_{r}} \in Y^{*}$ is denoted by $|w|$ and its degree equals to $s_{1}+\ldots+s_{r}$.

The quasi-symmetric function $\mathrm{F}_{w}$, of depth $r=|w|$ and of degree (or weight) $s_{1}+\ldots+s_{r}$, is defined by

$$
\mathrm{F}_{w}(\underline{t})=\sum_{n_{1}>\ldots>n_{r}>0} t_{n_{1}}^{s_{1}} \ldots t_{n_{r}}^{s_{r}}
$$

In particular, $\mathrm{F}_{y_{1}^{k}}=\eta_{k}$ and $\mathrm{F}_{y_{k}}=\psi_{k}$. The functions $\left\{\mathrm{F}_{y_{1}^{k}}\right\}_{k \geq 0}$ are linearly independent and integrating differential equation (17) shows that functions $\mathrm{F}_{y_{1}^{k}}$ and $\mathrm{F}_{y_{k}}$ are linked by the formula

$$
\sum_{k \geq 0} \mathrm{~F}_{y_{1}^{k}} z^{k}=\exp \left(-\sum_{k \geq 1} \mathrm{~F}_{y_{k}} \frac{(-z)^{k}}{k}\right)
$$

Every $\mathrm{H}_{w}(N)$ can be obtained by specializing, in the quasi-symmetric function $\mathrm{F}_{w}$, the variables $\left\{t_{i}\right\}_{i \geq 1}$ as follows [41]

$$
\forall N \geq i \geq 1, t_{i}=1 / i \text { and } \forall i>N, t_{i}=0 .
$$


In the same way, for $w \in Y^{*}-y_{1} Y^{*}$, the convergent polyzêta $\zeta(w)$ can be obtained by specializing, in $\mathrm{F}_{w}$, the variables $\left\{t_{i}\right\}_{i \geq 1}$ as follows [41]

$$
\forall N \geq i \geq 1, \quad t_{i}=1 / i .
$$

The notation $\mathrm{F}_{w}$ is extended by linearity to all polynomials over $Y$.

If $u, v \in Y^{*}$, of length $r, s$ and of weight ${ }^{6} p, q$ respectively, $\mathrm{F}_{u} \downarrow v$ is a quasisymmetric function of depth $r+s$ and of weight $p+q$, and $\mathrm{F}_{u \downarrow v}=\mathrm{F}_{u} \mathrm{~F}_{v}$, where $\omega$ is the quasi-shuffle product ${ }^{7}[41]$. Hence,

$$
\begin{aligned}
\forall u, v \in Y^{*}, & \mathrm{H}_{u} \text { шv }=\mathrm{H}_{u} \mathrm{H}_{v}, \\
\Rightarrow \quad \forall u, v \in Y^{*}-y_{1} Y^{*}, & \zeta(u \varpi v)=\zeta(u) \zeta(v) .
\end{aligned}
$$

Remarkable identity (18) can be then seen as

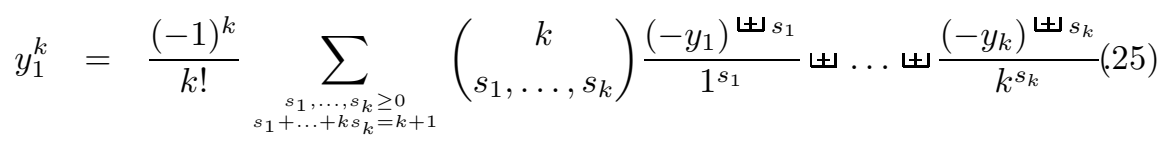

\subsubsection{Iterated integrals and polylogarithms}

Let $X$ be the finite alphabet $\left\{x_{0}, x_{1}\right\}$ equipped with the order $x_{0}<x_{1}{ }^{8}$. Let

$$
\mathcal{C}:=\mathbb{C}\left[z, \frac{1}{z}, \frac{1}{1-z}\right] \quad \text { and } \quad \mathcal{G}:=\left\{z, \frac{1}{z}, \frac{z-1}{z}, \frac{z}{z-1}, \frac{1}{1-z}, 1-z\right\} .
$$

This ring $\mathcal{C}$ is invariant under differentiation and under the homographic transformations belonging to the group $\mathcal{G}$ whose elements commute the singularities $\{0,1,+\infty\}$.

The iterated integral over $\omega_{0}, \omega_{1}$ associated to the word $w=x_{i_{1}} \cdots x_{i_{k}}$ over $X^{*}$ (the monoid generated by $X$ ) and along the integration path $z_{0} \rightsquigarrow z$ is the following multiple integral defined by

$$
\int_{z_{0} \rightsquigarrow z} \omega_{i_{1}} \cdots \omega_{i_{k}}=\int_{z_{0}}^{z} \omega_{i_{1}}\left(t_{1}\right) \int_{z_{0}}^{t_{1}} \omega_{i_{2}}\left(t_{2}\right) \ldots \int_{z_{0}}^{t_{r-2}} \omega_{i_{r}}\left(t_{r-1}\right) \int_{z_{0}}^{t_{r-1}} \omega_{i_{r}}\left(t_{r}\right),
$$

where $t_{1} \cdots t_{r-1}$ is a subdivision of the path $z_{0} \rightsquigarrow z$. In a shortened notation, we denote this integral by $\alpha_{z_{0}}^{z}(w)$ and $^{9} \alpha_{z_{0}}^{z}(\epsilon)=1$. One can check that the polylogarithm $\mathrm{Li}_{s_{1}, \ldots, s_{r}}$ is also the value of the iterated integral over $\omega_{0}, \omega_{1}$ and along the integration path $0 \rightsquigarrow z[29,31]$ :

$$
\operatorname{Li}_{w}(z)=\alpha_{0}^{z}\left(x_{0}^{s_{1}-1} x_{1} \ldots x_{0}^{s_{r}-1} x_{1}\right) .
$$

The definition of polylogarithms is extended over the words $w \in X^{*}$ by putting $\operatorname{Li}_{x_{0}}(z):=\log z$. The $\left\{\operatorname{Li}_{w}\right\}_{w \in X^{*}}$ are $\mathcal{C}$-linearly independent $[35,32]$. The

\footnotetext{
${ }^{6}$ The weight is as in Equation (19).

${ }^{7}$ See Annexe A, for the study of the Hopf algebra of $\boldsymbol{t}+$ which is not included in [45].

${ }^{8}$ In all the sequel, we follow the notations of $[2,45]$.

${ }^{9}$ Here, $\epsilon$ stands for the empty word over $X$.
} 
functions $\mathrm{P}_{w}(z):=(1-z)^{-1} \mathrm{Li}_{w}(z), w \in X^{*}$, are also $\mathbb{C}$-linearly independent. Since, for $w \in Y^{*}, \mathrm{P}_{w}$ is the ordinary generating function of $\left\{\mathrm{H}_{w}(N)\right\}_{N \geq 0}[37]$ :

$$
\mathrm{P}_{w}(z)=\sum_{N \geq 0} \mathrm{H}_{w}(N) z^{N}
$$

then, as a consequence of the classical isomorphism between convergent Taylor series and their associated sums, the harmonic sums $\left\{\mathrm{H}_{w}\right\}_{w \in Y^{*}}$ are also $\mathbb{C}$ linearly independent. Firstly, ker $\mathrm{P}=\{0\}$ and $\operatorname{ker} \mathrm{H}=\{0\}$, and secondly, $\mathrm{P}$ is a morphism for the Hadamard product :

$$
\mathrm{P}_{u}(z) \odot \mathrm{P}_{v}(z)=\sum_{N \geq 0} \mathrm{H}_{u}(N) \mathrm{H}_{v}(N) z^{N}=\sum_{N \geq 0} \mathrm{H}_{u} \amalg v(N) z^{N}=\mathrm{P}_{u \amalg v}(z) .
$$

Proposition 1 ([37]). Extended by linearity, the following maps are isomorphism of algebras

$$
\begin{aligned}
\mathrm{P}:(\mathbb{C}\langle Y\rangle,+ \pm) & \longrightarrow\left(\mathbb{C}\left\{\mathrm{P}_{w}\right\}_{w \in Y^{*}}, \odot\right), \\
u & \longmapsto \mathrm{P}_{u}, \\
\mathrm{H}:(\mathbb{C}\langle Y\rangle,+ \pm) & \longrightarrow\left(\mathbb{C}\left\{\mathrm{H}_{w}\right\}_{w \in Y^{*}}, .\right), \\
u & \longmapsto \mathrm{H}_{u}=\left\{\mathrm{H}_{u}(N)\right\}_{N \geq 0} .
\end{aligned}
$$

Studying the equivalence between action of $\left\{(1-z)^{l}\right\}_{l \in \mathbb{Z}}$ over $\left\{\mathrm{P}_{w}(z)\right\}_{w \in Y^{*}}$ and that of $\left\{N^{k}\right\}_{k \in \mathbb{Z}}$ over $\left\{\mathrm{H}_{w}(N)\right\}_{w \in Y^{*}}$ (see [12]), we have

Theorem 1 ([39]). The Hadamard $\mathcal{C}$-algebra of $\left\{\mathrm{P}_{w}\right\}_{w \in Y^{*}}$ can be identified with that of $\left\{\mathrm{P}_{l}\right\}_{l \in \mathcal{L} y n Y}$. In the same way, the algebra of harmonic sums $\left\{\mathrm{H}_{w}\right\}_{w \in Y^{*}}$ with polynomial coefficients can be identified with that of $\left\{\mathrm{H}_{l}\right\}_{l \in \mathcal{L} y n Y}$.

By Identity (25) and by applying the isomorphism $\mathrm{H}$ on the set of Lyndon words $\left\{y_{r}\right\}_{1 \leq r \leq k}$, we obtain $\mathrm{H}_{y_{1}^{k}}$ as polynomials in $\left\{\mathrm{H}_{y_{r}}\right\}_{1 \leq r \leq k}$ (which are algebraically independent), and

$$
\mathrm{H}_{y_{1}^{k}}=\sum_{\substack{s_{1}, \ldots, s_{k} \geq 0 \\ s_{1}+\ldots, k s_{k}=k+1}} \frac{(-1)^{k}}{s_{1} ! \ldots s_{k} !}\left(-\frac{\mathrm{H}_{y_{1}}}{1}\right)^{s_{1}} \ldots\left(-\frac{\mathrm{H}_{y_{k}}}{k}\right)^{s_{k}} .
$$

\subsection{Results à la Abel for generating series of harmonic sums and of polylogarithms}

\subsubsection{Generating series of harmonic sums and of polylogarithms}

Let $\mathrm{H}(N)$ be the noncommutative generating series of $\left\{\mathrm{H}_{w}(N)\right\}_{w \in Y^{*}}[37]$ :

$$
\mathrm{H}(N):=\sum_{w \in Y^{*}} \mathrm{H}_{w}(N) w .
$$

Let $\left\{\Sigma_{w}\right\}_{w \in Y^{*}},\left\{\check{\Sigma}_{w}\right\}_{w \in Y^{*}}$ be respectively a PBW basis of the envelopping algebra $\mathcal{U}\left(\mathcal{L}_{i} e_{\mathbb{Q}}\langle Y\rangle\right)$ and the quasi-shuffle algebra $(\mathbb{Q}\langle Y\rangle$, $\leftarrow$ ) (viewed as a $\mathbb{Q}$ module) on duality such that $\left\{\Sigma_{l}\right\}_{l \in \mathcal{L} y n X},\left\{\bar{\Sigma}_{l}\right\}_{l \in \mathcal{L} y n X}$ are respectively a basis of $\mathcal{L} i e_{\mathbb{Q}}\langle Y\rangle$ and a transcendence basis of $(\mathbb{Q}\langle Y\rangle, \boldsymbol{\omega})$ (see Annexe A). 
Theorem 2 (Factorization of $\mathrm{H})$. Let

$$
\mathrm{H}_{\mathrm{reg}}(N):=\prod_{l \in \mathcal{L} y n Y-\left\{y_{1}\right\}}^{\searrow} e^{\mathrm{H}_{\check{\Sigma}_{l}}(N) \Sigma_{l}} .
$$

Then $\mathrm{H}(N)=e^{\mathrm{H}_{y_{1}}(N) y_{1}} \mathrm{H}_{\text {reg }}(N)$.

Proof. See Annexe A.

For $l \in \mathcal{L} y n Y-\left\{y_{1}\right\}$, the polynomial $\Sigma_{l}$ is a finite combination of words in $Y^{*}-y_{1} Y^{*}$. Then we can state the following

Definition 1. We set $Z_{\amalg}:=\mathrm{H}_{\mathrm{reg}}(\infty)$.

The noncommutative generating series of polylogarithms $[35,32]$

$$
\mathrm{L}:=\sum_{w \in X^{*}} \mathrm{Li}_{w} w
$$

satisfies Drinfel'd's differential equation $(D E)$ of Example 1

$$
d \mathrm{~L}=\left(x_{0} \omega_{0}+x_{1} \omega_{1}\right) \mathrm{L}
$$

with boundary condition $[15,16]$

$$
\mathrm{L}(\varepsilon) \underset{\varepsilon \rightarrow 0^{+}}{\widetilde{x^{2}}} e^{x_{0} \log \varepsilon} .
$$

This enables us to prove that $\mathrm{L}$ is the exponential of a Lie series ${ }^{10}[35,32]$. Hence,

Proposition 2 (Logarithm of L, [38]). Let $\pi_{1}(w)$ is the following Lie series

$$
\pi_{1}(w)=\sum_{k \geq 1} \frac{(-1)^{k-1}}{k} \sum_{u_{1}, \ldots, u_{k} \in X^{+}}\left\langle w \mid u_{1} \omega \ldots ш u_{k}\right\rangle u_{1} \ldots u_{k} .
$$

Then

$$
\begin{aligned}
\log \mathrm{L}(z) & =\sum_{k \geq 1} \frac{(-1)^{k-1}}{k} \sum_{u_{1}, \ldots, u_{k} \in X^{+}} \operatorname{Li}_{u_{1}} ш \ldots w u_{k}(z) u_{1} \ldots u_{k} \\
& =\sum_{w \in X^{*}} \operatorname{Li}_{w}(z) \pi_{1}(w) .
\end{aligned}
$$

Applying a theorem of Ree [44, 45], L satisfies Friedrichs criterion [35, 32] :

$$
\begin{aligned}
\forall u, v \in X^{*}, & \operatorname{Li}_{u ш v}=\mathrm{Li}_{u} \mathrm{Li}_{v}, \\
\Rightarrow \quad \forall u, v \in x_{0} X^{*} x_{1}, & \zeta(u ш v)=\zeta(u) \zeta(v) .
\end{aligned}
$$

\footnotetext{
${ }^{10}$ i.e., $\mathrm{L}$ is goup-like for the co-product $\Delta_{\sqcup}: \Delta_{\amalg}(\mathrm{L})=\mathrm{L} \otimes \mathrm{L}$.
} 
Proposition 3 (Successive differentiation of $\mathrm{L},[38]$ ). For any $l \in \mathbb{N}$, let

$$
P_{l}(z)=\sum_{\operatorname{wgt}(\mathbf{r})=l} \sum_{w \in X^{\operatorname{deg}(\mathbf{r})}} \prod_{i=1}^{\operatorname{deg}(\mathbf{r})}\left(\begin{array}{c}
\sum_{j=1}^{i} r_{i}+j-1 \\
r_{i}
\end{array}\right) \tau_{\mathbf{r}}(w) \in \mathcal{C}\langle X\rangle,
$$

where, for any $w=x_{i_{1}} \cdots x_{i_{k}}$ and $\mathbf{r}=\left(r_{1}, \ldots, r_{k}\right)$ of degree $\operatorname{deg}(\mathbf{r})=k$ and of weight $\operatorname{wgt}(\mathbf{r})=k+r_{1}+\cdots+r_{k}$, the polynomial $\tau_{\mathbf{r}}(w)=\tau_{r_{1}}\left(x_{i_{1}}\right) \cdots \tau_{r_{k}}\left(x_{i_{k}}\right)$ is defined by

$$
\forall r \in \mathbb{N}, \quad \tau_{r}\left(x_{0}\right)=\partial^{r} \frac{x_{0}}{z}=\frac{-r ! x_{0}}{(-z)^{r+1}} \quad \text { and } \quad \tau_{r}\left(x_{1}\right)=\partial^{r} \frac{x_{1}}{1-z}=\frac{r ! x_{1}}{(1-z)^{r+1}} .
$$

Denoting $\partial=d / d z$, we have $\partial^{l} \mathrm{~L}(z)=P_{l}(z) \mathrm{L}(z)$.

Let $\left\{\check{S}_{l}\right\}_{l \in \mathcal{L} y n X}$ be the transcendence basis of the shuffle algebra $(\mathbb{Q}\langle X\rangle, \omega)$ and $\left\{\breve{S}_{w}\right\}_{w \in X^{*}}$ be the associated completed basis of the shuffle algebra $(\mathbb{Q}\langle X\rangle, w)$ (viewed as a $\mathbb{Q}$-module). They are defined as follows [45]

$$
\begin{aligned}
& \check{S}_{1_{X^{*}}}=\quad 1 \quad \text { for } l=1_{X^{*}} \\
& \check{S}_{l}=\quad x \check{S}_{u}, \quad \text { for } l=x u \in \mathcal{L} y n X, \\
& \check{S}_{w}=\frac{\check{S}_{l_{1}}^{\amalg i_{1}} \omega \ldots \omega \check{S}_{l_{k}}^{\amalg i_{k}}}{i_{1} ! \ldots i_{k} !} \text { for } w=l_{1}^{i_{1}} \ldots l_{k}^{i_{k}}, l_{1}>\ldots>l_{k} \text {. }
\end{aligned}
$$

Let $\left\{S_{w}\right\}_{w \in Y^{*}}$ be the PBW basis of the envelopping algebra $\mathcal{U}\left(\mathcal{L}_{i} e_{\mathbb{Q}}\langle X\rangle\right)$ in duality with the basis $\left\{\check{S}_{w}\right\}_{w \in Y^{*}}$ and $\left\{S_{l}\right\}_{l \in \mathcal{L} y n X}$ is then the basis of the Lie algebra $\mathcal{L i e}_{\mathbb{Q}}\langle X\rangle[45]$.

Theorem 3 (Factorization of L, [35, 32]). Let

$$
\mathrm{L}_{\mathrm{reg}}:=\prod_{l \in \mathcal{L} y n X-X}^{\searrow} e^{\mathrm{Li}_{S_{l}} \check{S}_{l}} .
$$

Then $\mathrm{L}(z)=e^{-x_{1} \log (1-z)} \mathrm{L}_{\mathrm{reg}}(z) e^{x_{0} \log z}$.

For $l \in \mathcal{L} y n X-X$, the polynomial $S_{l}$ is a finite combination of words in $x_{0} X^{*} x_{1}$. Then we can state the following

Definition $2([35,32])$. We set $Z_{山}:=\mathrm{L}_{\mathrm{reg}}(1)$.

In the definitions 1 and 2 only convergent polyzêtas arise and these noncommutative generating series will induce, in Section 3.1, two algebra morphisms of regularization as shown in the theorems 8 and 9 respectively. Hence, these power series are quite different of those given in [22] or in [43] (the last is based on [4], see [8]) needing a regularization process. 


\subsubsection{Asymptotic expansions by noncommutative generating series and regularized Chen generating series}

Let $\rho_{1-z}, \rho_{1-\frac{1}{z}}$ and $\rho_{\frac{1}{z}}[36,32]$ be three monoid morphisms verifying

$$
\begin{array}{rll}
\rho_{1-z}\left(x_{0}\right)=-x_{1} & \text { and } & \rho_{1-z}\left(x_{1}\right)=-x_{0}, \\
\rho_{1-1 / z}\left(x_{0}\right)=-x_{0}+x_{1} & \text { and } & \rho_{1-1 / z}\left(x_{1}\right)=-x_{0} \\
\rho_{1 / z}\left(x_{0}\right)=-x_{0}+x_{1} & \text { and } & \rho_{1 / z}\left(x_{1}\right)=x_{1} .
\end{array}
$$

Using homographic transformations belonging to the group $\mathcal{G}$, one has [36, 32]

$$
\begin{aligned}
\mathrm{L}(1-z) & =e^{x_{0} \log (1-z)} \rho_{1-z}\left[\mathrm{~L}_{\mathrm{reg}}(z)\right] e^{-x_{1} \log z} Z_{\uplus}, \\
\mathrm{L}(1-1 / z) & =e^{x_{0} \log (1-z)} \rho_{1-\frac{1}{z}}\left[\mathrm{~L}_{\mathrm{reg}}(z)\right] e^{-x_{1} \log z} \rho_{1-1 / z}\left(Z_{\uplus}^{-1}\right) e^{\mathrm{i} \pi x_{0}} \\
\mathrm{~L}(1 / z) & =e^{-x_{1} \log (1-z)} \rho_{1 / z}\left[\mathrm{~L}_{\mathrm{reg}}(z)\right] e^{\left(-x_{0}+x_{1}\right) \log z} \rho_{1 / z}\left(Z_{\amalg}^{-1}\right) e^{\mathrm{i} \pi x_{1}} Z_{\sqcup}
\end{aligned}
$$

Thus, (35) and (44) yield [36, 32]

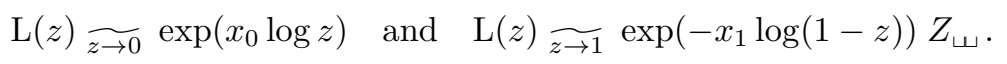

Let us call $\mathrm{LI}_{\mathcal{C}}$ the smallest algebra containing $\mathcal{C}$, closed under derivation and under integration with respect to $\omega_{0}$ and $\omega_{1}$. It is the $\mathcal{C}$-module generated by the polylogarithms $\left\{\mathrm{Li}_{w}\right\}_{w \in X^{*}}$.

Let $\pi_{Y}: \operatorname{LI}_{\mathcal{C}}\langle\langle X\rangle\rangle \longrightarrow \operatorname{LI}_{\mathcal{C}}\left\langle\langle Y\rangle\right.$ be a projector such that for any $f \in \mathrm{LI}_{\mathcal{C}}$ and $w \in X^{*}, \pi_{Y}\left(f w x_{0}\right)=0$. Then [39]

$$
\Lambda(z)=\pi_{Y} \mathrm{~L}(z) \underset{z \rightarrow 1}{\underset{ }{2}} \exp \left(y_{1} \log \frac{1}{1-z}\right) \pi_{Y} Z_{\amalg} .
$$

Since the coefficient of $z^{N}$ in the ordinary Taylor expansion of $\mathrm{P}_{y_{1}^{k}}$ is $\mathrm{H}_{y_{1}^{k}}(N)$ then let

$$
\begin{aligned}
& \operatorname{Mono}(z):=e^{-\left(x_{1}+1\right) \log (1-z)}=\sum_{k \geq 0} \mathrm{P}_{y_{1}^{k}}(z) y_{1}^{k} \\
& \text { Const }:=\sum_{k \geq 0} \mathrm{H}_{y_{1}^{k}} y_{1}^{k}=\exp \left(-\sum_{k \geq 1} \mathrm{H}_{y_{k}} \frac{\left(-y_{1}\right)^{k}}{k}\right) .
\end{aligned}
$$

Proposition 4 ([39]). We have

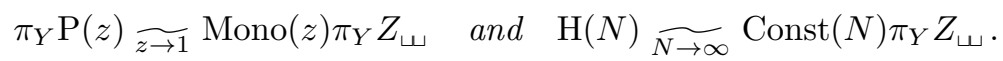

Proof. Let $\mu$ be the morphism verifying $\mu\left(x_{0}\right)=x_{1}$ and $\mu\left(x_{1}\right)=x_{0}$. Then, by Theorem 3, the noncommutative generating series of $\left\{\mathrm{P}_{w}\right\}_{w \in X^{*}}$ is given by

$$
\begin{aligned}
\mathrm{P}(z) & =(1-z)^{-1} \mathrm{~L}(z) \\
& =e^{-\left(x_{1}+1\right) \log (1-z)} \mathrm{L}_{\mathrm{reg}}(z) e^{x_{0} \log z} \\
& =e^{x_{0} \log z} \mu\left[\mathrm{L}_{\mathrm{reg}}(1-z)\right] e^{-\left(x_{1}+1\right) \log (1-z)} Z_{\amalg} \\
& =e^{x_{0} \log z} \mu\left[\mathrm{L}_{\mathrm{reg}}(1-z)\right] \operatorname{Mono}(z) Z_{\amalg} .
\end{aligned}
$$

Thus, $\mathrm{P}(z) \underset{z \rightarrow 0}{\widetilde{1}} e^{x_{0} \log z}$ and $\mathrm{P}(z) \underset{z \rightarrow 1}{\widetilde{M}} \operatorname{Mono}(z) Z_{w}$ yielding the expected results. 
As consequence of (48)-(50) and of Proposition 4, one gets

Theorem 4 (à la Abel, [39]).

$$
\lim _{z \rightarrow 1} \exp \left(y_{1} \log \frac{1}{1-z}\right) \Lambda(z)=\lim _{N \rightarrow \infty} \exp \left(\sum_{k \geq 1} \mathrm{H}_{y_{k}}(N) \frac{\left(-y_{1}\right)^{k}}{k}\right) \mathrm{H}(N)=\pi_{Y} Z_{\uplus} .
$$

Therefore, the knowledge of the ordinary Taylor expansion at 0 of the polylogarithmic functions $\left\{\mathrm{P}_{w}(1-z)\right\}_{w \in X^{*}}$ gives

Theorem 5 ([12]). For all $g \in \mathcal{C}\left\{\mathrm{P}_{w}\right\}_{w \in Y^{*}}$, there exists algorithmically computable $c_{j} \in \mathbb{C}, \alpha_{j} \in \mathbb{Z}, \beta_{j} \in \mathbb{N}$ and $b_{i} \in \mathbb{C}, \eta_{i} \in \mathbb{Z}, \kappa_{i} \in \mathbb{N}$ such that

$g(z) \underset{z \rightarrow 1}{\widetilde{1}} \sum_{j=0}^{+\infty} c_{j}(1-z)^{\alpha_{j}} \log ^{\beta_{j}}(1-z) \quad$ and $\quad\left[z^{n}\right] g(z) \underset{N \rightarrow+\infty}{\sim} \sum_{i=0}^{+\infty} b_{i} n^{\eta_{i}} \log ^{\kappa_{i}}(n)$.

Definition 3. Let $\mathcal{Z}$ be the $\mathbb{Q}$-algebra generated by convergent polyzêtas and let $\mathcal{Z}^{\prime}$ be the $e^{11} \mathbb{Q}[\gamma]$-algebra generated by $\mathcal{Z}$.

Corollary 1 ([12]). There exists algorithmically computable $c_{j} \in \mathcal{Z}, \alpha_{j} \in$ $\mathbb{Z}, \beta_{j} \in \mathbb{N}$ and $b_{i} \in \mathcal{Z}^{\prime}, \kappa_{i} \in \mathbb{N}, \eta_{i} \in \mathbb{Z}$ such that

$$
\begin{array}{lll}
\forall w \in Y^{*}, & \mathrm{P}_{w}(z) \sim \sum_{j=0}^{+\infty} c_{j}(1-z)^{\alpha_{j}} \log ^{\beta_{j}}(1-z) & \text { for } \quad z \rightarrow 1, \\
\forall w \in Y^{*}, & \mathrm{H}_{w}(N) \sim \sum_{i=0}^{+\infty} b_{i} N^{\eta_{i}} \log ^{\kappa_{i}}(N) & \text { for } \quad N \rightarrow+\infty .
\end{array}
$$

The Chen generating series along the path $z_{0} \rightsquigarrow z$, associated to $\omega_{0}, \omega_{1}$ is the following

$$
S_{z_{0} \rightsquigarrow z}:=\sum_{w \in X^{*}}\langle S \mid w\rangle w \quad \text { with }\langle S \mid w\rangle=\alpha_{z_{0}}^{z}(w)
$$

which solves the differential equation (34) with the initial condition $S_{z_{0} \rightsquigarrow z_{0}}=1$. Thus, $S_{z_{0} \rightsquigarrow z}$ and $\mathrm{L}(z) \mathrm{L}\left(z_{0}\right)^{-1}$ satisfy the same differential equation taking the same value at $z_{0}$ and

$$
S_{z_{0} \rightsquigarrow z}=\mathrm{L}(z) \mathrm{L}\left(z_{0}\right)^{-1} .
$$

Any Chen generating series $S_{z_{0} \rightsquigarrow z}$ is group like [44] and depends only on the homotopy class of $z_{0} \rightsquigarrow z[10]$. The product of $S_{z_{1} \rightsquigarrow z_{2}}$ and $S_{z_{0} \rightsquigarrow z_{1}}$ is the Chen generating series

$$
S_{z_{0} \rightsquigarrow z_{2}}=S_{z_{1} \rightsquigarrow z_{2}} S_{z_{0} \rightsquigarrow z_{1}}
$$

\footnotetext{
${ }^{11}$ Here, $\gamma$ stands for the Euler constant $\gamma=.57721566490153286060651209008240243 \ldots$
} 


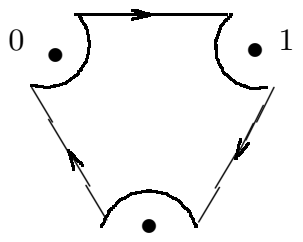

$\infty$

Figure 1: Hexagonal path

Let $\varepsilon \in] 0,1\left[\right.$ and let $z_{i}=\varepsilon \exp \left(\mathrm{i} \theta_{i}\right)$, for $i=0$ or 1 . We set $\theta=\theta_{1}-\theta_{0}$. Let $\Gamma_{0}(\varepsilon, \theta)$ (resp. $\Gamma_{1}(\varepsilon, \theta)$ ) be the path turning around 0 (resp. 1) in the positive direction from $z_{0}$ to $z_{1}$. By induction on the length of $w$, one has

$$
\left|\left\langle S_{\Gamma_{i}(\varepsilon, \theta)} \mid w\right\rangle\right|=(2 \varepsilon)^{|w| x_{i}} \theta^{|w| \mid} /|w| !,
$$

where, $|w|$ denotes the lenghth of $w$ and $|w|_{x_{i}}$, denotes the number of occurrences of letter $x_{i}$ in $w$, for $i=0,1$. For $\varepsilon \rightarrow 0^{+}$, these estimations yield

$$
S_{\Gamma_{i}(\varepsilon, \theta)}=e^{\mathrm{i} \theta x_{i}}+o(\varepsilon) .
$$

In particular, if $\Gamma_{0}(\varepsilon)$ (resp. $\Gamma_{1}(\varepsilon)$ ) is a circular path of radius $\varepsilon$ turning around 0 (resp. 1) in the positive direction, starting at $z=\varepsilon$ (resp. $1-\varepsilon$ ), then, by the noncommutative residu theorem $[35,32]$, we get

$$
S_{\Gamma_{0}(\varepsilon)}=e^{2 \mathrm{i} \pi x_{0}}+o(\varepsilon) \text { and } S_{\Gamma_{1}(\varepsilon)}=e^{-2 \mathrm{i} \pi x_{1}}+o(\varepsilon) .
$$

Finally, the asymptotic behaviors of L on (47) give

Proposition $5([32,35])$. We have $S_{\varepsilon \rightsquigarrow 1-\varepsilon} \underset{\varepsilon \rightarrow 0^{+}}{\widetilde{x_{1}}} e^{-x_{1} \log \varepsilon} Z_{\amalg} e^{-x_{0} \log \varepsilon}$.

In other terms, $Z_{\amalg}$ is the regularized Chen generating series $S_{\varepsilon \rightsquigarrow 1-\varepsilon}$ of diffferential forms $\omega_{0}$ and $\omega_{1}: Z_{\omega}$ is the noncommutative generating series of the finite parts of the coefficients of the Chen generating series $e^{x_{1} \log \varepsilon} S_{\varepsilon \rightsquigarrow 1-\varepsilon} e^{x_{0} \log \varepsilon}$ : the concatenation of $e^{x_{0} \log \varepsilon}$ and then $S_{\varepsilon \rightsquigarrow 1-\varepsilon}$ and finally, $e^{x_{1} \log \varepsilon}$.

Proposition 6. Let $\rho_{1-1 / z}$ be the morphism is given in Section 2.2.2. We have

$$
\prod_{\substack{l \in \mathcal{L} y n X \\ l \neq x_{0}, x_{1}}}^{\searrow} e^{\zeta(\check{l}) l}=e^{\mathrm{i} \pi x_{0}} \prod_{\substack{l \in \mathcal{L} y n X \\ l \neq x_{0}, x_{1}}}^{\searrow} e^{\zeta(\check{l}) \rho_{1-1 / z}(l)} e^{\mathrm{i} \pi\left(-x_{0}+x_{1}\right)} \prod_{\substack{l \in \mathcal{L} y y_{X} \\ l \neq x_{0}, x_{1}}}^{\searrow} e^{\zeta(\check{l}) \rho_{1-1 / z}^{2}(l)} e^{-\mathrm{i} \pi x_{1}} .
$$

Proof. Following the hexagonal path given in Figure 1, one has [36, 32]

$$
\left(S_{\varepsilon \rightsquigarrow 1-\varepsilon} e^{\mathrm{i} \pi x_{0}}\right) \rho_{1-1 / z}\left(S_{\varepsilon \rightsquigarrow 1-\varepsilon} e^{\mathrm{i} \pi x_{0}}\right) \rho_{1-1 / z}^{2}\left(S_{\varepsilon \rightsquigarrow 1-\varepsilon} e^{\mathrm{i} \pi x_{0}}\right)=1+O(\sqrt{\varepsilon}) .
$$

By Proposition 5, it follows the hexagonal relation [15, 16, 36, 32] which is

$$
\begin{aligned}
& Z_{\sqcup} e^{\mathrm{i} \pi x_{0}} \rho_{1-1 / z}\left(Z_{\sqcup}\right) e^{\mathrm{i} \pi\left(-x_{0}+x_{1}\right)} \rho_{1-1 / z}^{2}\left(Z_{\sqcup}\right) e^{-\mathrm{i} \pi x_{1}}=1, \\
\Longleftrightarrow \quad & e^{\mathrm{i} \pi x_{0}} \rho_{1-1 / z}\left(Z_{\sqcup}\right) e^{\mathrm{i} \pi\left(-x_{0}+x_{1}\right)} \rho_{1-1 / z}^{2}\left(Z_{\sqcup}\right) e^{-\mathrm{i} \pi x_{1}}=Z_{\sqcup}^{-1} .
\end{aligned}
$$

It follows then the expected result. 


\subsection{Indiscernability over a class of formal power series}

\subsubsection{Residual calculus and representative series}

Definition 4. Let $S \in \mathbb{Q}\langle\langle X\rangle\rangle$ and let $P \in \mathbb{Q}\langle X\rangle$.

The left residual (resp. right residual) of $S$ by $P$, is the formal power series $P \triangleleft S$ (resp. $S \triangleright P)$ in $\mathbb{Q}\langle\langle X\rangle\rangle$ defined by :

$$
\langle P \triangleleft S \mid w\rangle=\langle S \mid w P\rangle \quad(\text { resp. } \quad\langle S \triangleright P \mid w\rangle=\langle S \mid P w\rangle) .
$$

We straightforwardly get, for any $P, Q \in \mathbb{Q}\langle X\rangle$ :

$$
P \triangleleft(Q \triangleleft S)=P Q \triangleleft S,(S \triangleright P) \triangleright Q=S \triangleright P Q,(P \triangleleft S) \triangleright Q=P \triangleleft(S \triangleright Q)
$$

In case $x, y \in X$ and $w \in X^{*}$, we get $x \triangleleft(w y)=\delta_{x, y} w$ and $x w \triangleright y=\delta_{x, y} w$.

Lemma 1 (Reconstruction lemma). Let $S \in \mathbb{Q}\langle\langle X\rangle\rangle$. Then

$$
S=\langle S \mid \epsilon\rangle+\sum_{x \in X} x(S \triangleright x)=\langle S \mid \epsilon\rangle+\sum_{x \in X}(x \triangleleft S) x .
$$

Lemma 2. The left and right residuals by a letter $x$ are derivations in $(\mathbb{Q}\langle\langle X\rangle, \sqcup \sqcup)$ : $x \triangleleft(u \sqcup v)=(x \triangleleft u) \sqcup v+u ш(x \triangleleft v), \quad(u ш v) \triangleright x=(u \triangleright x) \sqcup v+u \sqcup(v \triangleright x)$.

Proof. Use the recursive definitions of the shuffle product.

Lemma 3. For any Lie polynomial $Q \in \mathcal{L} i e_{\mathbb{Q}}\langle X\rangle$, the linear maps " $Q \triangleleft$ " and " $Q$ " are derivations on $(\mathbb{Q}[\mathcal{L} y n X], \omega)$.

Proof. For any $l, l_{1}, l_{2} \in \mathcal{L} y n X$, we have

$$
\begin{gathered}
\hat{l} \triangleleft\left(l_{1} \sqcup l_{2}\right)=l_{1} \sqcup\left(\hat{l} \triangleleft l_{2}\right)+\left(\hat{l} \triangleleft l_{1}\right) \sqcup l_{2}=l_{1} \delta_{l_{2}, \hat{l}}+\delta_{l_{1}, \hat{l}} l_{2}, \\
\left(l_{1} \uplus l_{2}\right) \triangleright \hat{l}=l_{1} \sqcup\left(l_{2} \triangleright \hat{l}\right)+\left(l_{1} \triangleright \hat{l}\right) \sqcup l_{2}=l_{1} \delta_{l_{2}, \hat{l}}+\delta_{l_{1}, \hat{l}} l_{2} .
\end{gathered}
$$

Lemma 4. For any Lyndon word $l \in \mathcal{L} y n X$ and $\check{S}_{l}$ defined as in (39), one has

$$
x_{1} \triangleleft l=l \triangleright x_{0}=0 \quad \text { and } \quad x_{1} \triangleleft \check{S}_{l}=\check{S}_{l} \triangleright x_{0}=0 .
$$

Proof. Since $x_{1} \triangleleft$ and $\triangleright x_{0}$ are derivations and for any $l \in \mathcal{L} y n X-X$, the polynomial $\check{S}_{l}$ belongs to $x_{0} \mathbb{Q}\langle X\rangle x_{1}$ then it follows the expected results.

Theorem 6 (On representative series). The following properties are equivalent for any series $S \in \mathbb{Q}\langle\langle X\rangle\rangle$ :

1. The left $\mathbb{C}$-module $\operatorname{Res}_{g}(S)=\operatorname{span}\left\{w \triangleleft S \mid w \in X^{*}\right\}$ is finite dimensional.

2. The right $\mathbb{C}$-module $\operatorname{Res}_{d}(S)=\operatorname{span}\left\{S \triangleright w \mid w \in X^{*}\right\}$ is finite dimensional. 
3. There are matrices $\lambda \in \mathcal{M}_{1, n}(\mathbb{Q}), \eta \in \mathcal{M}_{n, 1}(\mathbb{Q})$ and a representation of $X^{*}$ in $\mathcal{M}_{n, n}$, such that

$$
S=\sum_{w \in X^{*}}[\lambda \mu(w) \eta] w=\lambda\left(\prod_{l \in \mathcal{L} y n X}^{\searrow} e^{\mu\left(S_{l}\right) \check{S}_{l}}\right) \eta .
$$

A series that safisfies the items of this theorem will be called representative series. This concept can be found in $[1,42,13]$. The two first items are in $[18,24]$. The third item can be deduced from $[9,11]$ for example and it was used to factorize first time, by Lyndon words, the output of bilinear and analytical dynamical systems respectively in $[26,27]$ and to study polylogarithms, hypergeometric functions and associated functions in [29, 31, 38]. The dimension of $\operatorname{Res}_{g}(S)$ is equal to that of $\operatorname{Res}_{d}(S)$, and to the minimal dimension of a representation satisfying the third point of Theorem 6 . This rank is then equal to the rank of the Hankel matrix of $S$, that is the infinite matrix $(\langle S \mid u v\rangle)_{u, v \in X}$ indexed by $X^{*} \times X^{*}$ and is also called Hankel rank of $S[18,24]$ :

Definition 5 ([18, 24]). The Hankel rank of a formal power series $S \in \mathbb{C}\langle\langle X\rangle\rangle$ is the dimension of the vector space

$$
\{S \triangleright \Pi \mid \Pi \in \mathbb{C}\langle X\rangle\}, \quad(\text { resp. } \quad\{\Pi \triangleleft S \mid \Pi \in \mathbb{C}\langle X\rangle\} .
$$

The triplet $(\lambda, \mu, \eta)$ is called a linear representation of $S$. We define the minimal representation ${ }^{12}$ of $S$ as being a representation of $S$ of minimal dimension.

For any proper series $S$, the following power series is called "star of $S$ "

$$
S^{*}=1+S+S^{2}+\ldots+S^{n}+\ldots
$$

Definition 6 ([2, 47]). A series $S$ is called rational if it belongs to the closure in $\mathbb{Q}\langle\langle X\rangle$ of the noncommutative polynomial algebra by sum, product and star operation of proper ${ }^{13}$ elements. The set of rational power series will be denoted by $\mathbb{Q}^{\mathrm{rat}}\langle\langle X\rangle$.

Lemma 5. For any noncommutative rational series (resp. polynomial) $R$ and for any polynomial $P$, the left and right residuals of $R$ by $P$ are rational (resp. polynomial).

Theorem 7 (Schützenberger, $[2,47])$. Any noncommutative power series is representative if and only if it is rational.

\subsubsection{Continuity and indiscernability}

Definition $7([25,39])$. Let $\mathcal{H}$ be a class of formal power series over $X$ and let $S \in \mathbb{C}\langle\langle X\rangle\rangle$.

\footnotetext{
${ }^{12}$ It can be shown that all minimal representations are isomorphics (see [2]).

${ }^{13} \mathrm{~A}$ series $S$ is said to be proper if $\langle S \mid \epsilon\rangle=0$.
} 
1. $S$ is said to be continuous ${ }^{14}$ over $\mathcal{H}$ if for any $\Phi \in \mathcal{H}$, the following sum, denoted $\langle S \| \Phi\rangle$, is convergent in norm

$$
\sum_{w \in X^{*}}\langle S \mid w\rangle\langle\Phi \mid w\rangle
$$

The set of continuous power series over $\mathcal{H}$ will be denoted by $\mathbb{C}^{\operatorname{cont}}\langle\langle X\rangle\rangle$.

2. $S$ is said to be indiscernable ${ }^{15}$ over $\mathcal{H}$ if and only if

$$
\forall \Phi \in \mathcal{H}, \quad\langle S \| \Phi\rangle=0 .
$$

Let $\rho$ be the monoid morphism verifying $\rho\left(x_{0}\right)=x_{1}$ and $\rho\left(x_{1}\right)=x_{0}$ and let $\hat{w}=\rho(\tilde{w})$, where $\tilde{w}$ is the mirror of $w$.

Lemma 6. Let $S \in \mathbb{C}^{\text {cont }}\langle\langle X\rangle\rangle$. If $\left\langle S \| Z_{\sqcup}\right\rangle=0$ then $\left\langle\hat{S} \| Z_{\omega}\right\rangle=0$, where

$$
\hat{S}:=\sum_{w \in X^{*}}\langle S \mid w\rangle \hat{w}
$$

Proof. For any $w \in x_{0} X^{*} x_{1}$, by "duality relation", one has (see $\left.[40,50,36]\right)$

$$
\zeta(\hat{w})=\zeta(w), \quad \text { or equivalently } \quad Z_{\uplus}=\hat{Z}_{\sqcup}:=\sum_{w \in X^{*}}\left\langle Z_{\varpi} \mid w\right\rangle \hat{w} .
$$

Using the fact

$$
\left\langle\hat{S} \| Z_{\sqcup}\right\rangle=\sum_{\hat{w} \in X^{*}}\langle S \mid \hat{w}\rangle\left\langle Z_{\sqcup} \mid \hat{w}\right\rangle=\sum_{w \in X^{*}}\langle S \mid w\rangle\left\langle Z_{\sqcup} \mid w\right\rangle,
$$

one gets finally the expected result.

Lemma 7. Let $\mathcal{H}$ be a monoid containing $\left\{e^{t x}\right\}_{x \in X}^{t \in \mathbb{C}}$. Let $S \in \mathbb{C}^{\operatorname{cont}}\langle\langle X\rangle\rangle$ being indiscernable over $\mathcal{H}$. Then for any $x \in X, x \triangleleft S$ and $S \triangleright x$ belong to $\mathbb{C}^{\operatorname{cont}}\langle\langle X\rangle\rangle$ and they are indiscernable over $\mathcal{H}$.

Proof. Let us calculate $\langle x \triangleleft S \| \Phi\rangle=\langle S \| \Phi x\rangle$ and $\langle S \triangleright x \| \Phi\rangle=\langle S \| x \Phi\rangle$. Since

$$
\lim _{t \rightarrow 0} \frac{e^{t x}-1}{t}=x \quad \text { and } \quad \lim _{t \rightarrow 0} \frac{e^{t x}-1}{t}=x
$$

then, for any $\Phi \in \mathcal{H}$, by uniform convergence, one has

$$
\begin{aligned}
& \langle S \| \Phi x\rangle=\left\langle S \| \lim _{t \rightarrow 0} \Phi \frac{e^{t x}-1}{t}\right\rangle=\lim _{t \rightarrow 0}\left\langle S \| \Phi \frac{e^{t x}-1}{t}\right\rangle, \\
& \langle S \| x \Phi\rangle=\left\langle S \| \lim _{t \rightarrow 0} \frac{e^{t x}-1}{t} \Phi\right\rangle=\lim _{t \rightarrow 0}\left\langle S \| \frac{e^{t x}-1}{t} \Phi\right\rangle .
\end{aligned}
$$

${ }^{14}$ See $[25,39]$, for a convergence criterion and an example of continuous generating series.

${ }^{15}$ Here, we adapt this notion developped in [25] via the residual calculus. 
Since $S$ is indiscernable over $\mathcal{H}$ then

$$
\begin{aligned}
& \langle S \| \Phi x\rangle=\lim _{t \rightarrow 0} \frac{1}{t}\left\langle S \| \Phi e^{t x}\right\rangle-\lim _{t \rightarrow 0} \frac{1}{t}\langle S \| \Phi\rangle=0, \\
& \langle S \| x \Phi\rangle=\lim _{t \rightarrow 0} \frac{1}{t}\left\langle S \| e^{t x} \Phi\right\rangle-\lim _{t \rightarrow 0} \frac{1}{t}\langle S \| \Phi\rangle=0 .
\end{aligned}
$$

Proposition 7. Let $\mathcal{H}$ be a monoid containing $\left\{e^{t x}\right\}_{x \in X}^{t \in \mathbb{C}}$. The formal power series $S \in \mathbb{C}^{\text {cont }}\langle\langle X\rangle\rangle$ is indiscernable over $\mathcal{H}$ if and only if $S=0$.

Proof. If $S=0$ then it is immediate that $S$ is indiscernable over $\mathcal{H}$. Conversely, if $S$ is indiscernable over $\mathcal{H}$ then by Lemma 7 , for any word $w \in X^{*}$, by induction on the length of $w, w \triangleleft S$ is indiscernable over $\mathcal{H}$ and then in particular,

$$
\left\langle w \triangleleft S \| \operatorname{Id}_{\mathcal{H}}\right\rangle=\langle S \mid w\rangle=0 .
$$

In other words, $S=0$.

\section{Group of associators : polynomial relations among convergent polyzêtas and identification of local coordinates}

\subsection{Generalized Euler constants and global regularization of polyzêtas}

\subsubsection{Three regularizations of divergent polyzêtas}

Theorem $8([33])$. Let $\zeta_{\mathbf{t +}}:(\mathbb{Q}\langle Y\rangle$, $\mathbf{\pm}) \rightarrow(\mathbb{R},$.$) be the morphism verifying$ the following properties

- for $u, v \in Y^{*}, \zeta_{\mathbf{L}}(u \leftarrow v)=\zeta_{\mathbf{\pm t}}(u) \zeta_{\mathbf{4 \pm}}(v)$,

- for all convergent word $w \in Y^{*}-y_{1} Y^{*}, \zeta_{\mathbf{4 +}}(w)=\zeta(w)$,

- $\zeta_{\text {L+I }}\left(y_{1}\right)=0$.

Then

$$
\sum_{w \in X^{*}} \zeta_{\mathbf{4}}(w) w=Z_{\amalg \downarrow}
$$

Corollary $2([33])$. For any $w \in X^{*}, \zeta_{\mathbf{4 +}}(w)$ belongs to the algebra $\mathcal{Z}$.

Theorem $9([33])$. Let $\zeta_{\amalg}:(\mathbb{Q}\langle X\rangle, \omega) \rightarrow(\mathbb{R},$.$) be the morphism verifying the$ following properties

- for $u, v \in X^{*}, \zeta_{\sqcup}(u \sqcup v)=\zeta_{\sqcup}(u) \zeta_{\sqcup}(v)$, 
- for all convergent word $w \in x_{0} X^{*} x_{1}, \zeta_{\omega}(w)=\zeta(w)$,

- $\zeta_{\amalg}\left(x_{0}\right)=\zeta_{\amalg}\left(x_{1}\right)=0$.

Then

$$
\sum_{w \in X^{*}} \zeta_{\varpi}(w) w=Z_{山} .
$$

Corollary 3 ([33]). For any $w \in Y^{*}, \zeta_{\omega}(w)$ belongs to the algebra $\mathcal{Z}$.

Definition 8. For any $w \in Y^{*}$, let $\gamma_{w}$ be the constant part ${ }^{16}$ of the asymptotic expasion, on the comparison scale $\left\{n^{a} \log ^{b}(n)\right\}_{a \in \mathbb{Z}, b \in \mathbb{N}}$, of $\mathrm{H}_{w}(n)$.

Let $Z_{\gamma}$ be the noncommutative generating series of $\left\{\gamma_{w}\right\}_{w \in Y^{*}}$ :

$$
Z_{\gamma}:=\sum_{w \in Y^{*}} \gamma_{w} w
$$

Definition 9. We set

$$
B\left(y_{1}\right):=\exp \left(-\sum_{k \geq 1} \gamma_{y_{k}} \frac{\left(-y_{1}\right)^{k}}{k}\right) \quad \text { and } \quad B^{\prime}\left(y_{1}\right):=e^{-\gamma y_{1}} B\left(y_{1}\right) .
$$

The power series $B^{\prime}\left(y_{1}\right)$ corresponds in fact to the mould ${ }^{17}$ Mono in [17] and to the $\Phi_{\text {corr }}$ in [43] (see also $[4,8]$ ). While the power series $B\left(y_{1}\right)$ corresponds to the Gamma Euler function with its product expansion,

$$
B\left(y_{1}\right)=\Gamma\left(y_{1}+1\right), \quad \frac{1}{\Gamma\left(y_{1}+1\right)}=e^{\gamma y_{1}} \prod_{n \geq 1}\left(1+\frac{y_{1}}{n}\right) e^{-\gamma / n} .
$$

Lemma 8 ([39]). Let $b_{n, k}\left(t_{1}, \ldots, t_{n-k+1}\right)$ be the (exponential) partial Bell polynomials in the variables $\left\{t_{l}\right\}_{l \geq 1}$ given by the exponential generating series

$$
\exp \left(u \sum_{l=0}^{\infty} t_{l} \frac{v^{l}}{l !}\right)=\sum_{n, k=0}^{\infty} b_{n, k}\left(t_{1}, \ldots, t_{n-k+1}\right) \frac{v^{n} u^{k}}{n !} .
$$

For any $m \geq 1$, let $t_{m}=(-1)^{m}(m-1) ! \gamma_{y_{m}}$. Then

$$
B\left(y_{1}\right)=1+\sum_{n \geq 1}\left(\sum_{k=1}^{n} b_{n, k}(\gamma,-\zeta(2), 2 \zeta(3), \ldots)\right) \frac{\left(-y_{1}\right)^{n}}{n !} .
$$

Since the ordinary generating series of the finite parts of coefficients of Const $(N)$ is nothing else but the power series $B\left(y_{1}\right)$, taking the constant part on either side of $\mathrm{H}(N) \widetilde{N \rightarrow \infty} \operatorname{Const}(N) \pi_{Y} Z_{山}$ (see Proposition 4), yields

Theorem 10 ([39]). We have $Z_{\gamma}=B\left(y_{1}\right) \pi_{Y} Z_{\amalg}$.

\footnotetext{
${ }^{16}$ i.e. $\gamma_{w}$ is the Euler-Mac Laurin constante of $\mathrm{H}_{w}(n)$.

${ }^{17}$ The readers can see why we have introduced the power series Mono $(z)$ in Proposition 4.
} 
Identifying the coefficients of $y_{1}^{k} w$ on either side using the identity ${ }^{18}[33]$

$$
\forall u \in X^{*} x_{1}, \quad x_{1}^{k} x_{0} u=\sum_{l=0}^{k} x_{1}^{l} \uplus\left(x_{0}\left[\left(-x_{1}\right)^{k-l} \uplus u\right]\right)
$$

and applying the morphism $\zeta_{\amalg}$ given in Theorem 9, we get [33]

$$
\forall u \in X^{*} x_{1}, \quad \zeta_{\sqcup}\left(x_{1}^{k} x_{0} u\right)=\zeta\left(x_{0}\left[\left(-x_{1}\right)^{k} \sqcup u\right]\right) .
$$

Corollary 4 ([39]). For $w \in x_{0} X^{*} x_{1}$, i.e. $w=x_{0} u$ and $\pi_{Y} w \in Y^{*}-y_{1} Y^{*}$, and for $k \geq 0$, the constant $\gamma_{\mathbf{\omega}}\left(x_{1}^{k} w\right)$ associated to the divergent polyzêta $\zeta\left(x_{1}^{k} w\right)$ is a polynomial of degree $k$ in $\gamma$ and with coefficients in $\mathcal{Z}$ :

$$
\gamma_{x_{1}^{k} w}=\sum_{i=0}^{k} \frac{\zeta\left(x_{0}\left[\left(-x_{1}\right)^{k-i} \uplus u\right]\right)}{i !}\left(\sum_{j=1}^{i} b_{i, j}(\gamma,-\zeta(2), 2 \zeta(3), \ldots)\right) .
$$

Moreover, for $l=0, . ., k$, the coefficient of $\gamma^{l}$ is of weight $|w|+k-l$.

In particular, for $s>1$, the constant $\gamma_{y_{1} y_{s}}$ associated to $\zeta\left(y_{1} y_{s}\right)$ is linear in $\gamma$ and with coefficients in $\mathbb{Q}[\zeta(2), \zeta(2 i+1)]_{0<i \leq(s-1) / 2}$.

Corollary 5 ([39]). The constant $\gamma_{x_{1}^{k}}$ associated to the divergent polyzêta $\zeta\left(x_{1}^{k}\right)$ is a polynomial of degree $k$ in $\gamma$ with coefficients in $\mathbb{Q}[\zeta(2), \zeta(2 i+1)]_{0<i \leq(k-1) / 2}$ :

$$
\gamma_{x_{1}^{k}}=\sum_{\substack{s_{1}, \ldots, s_{k} \geq 0 \\ s_{1}+\ldots+k s_{k}=k+1}} \frac{(-1)^{k}}{s_{1} ! \ldots s_{k} !}(-\gamma)^{s_{1}}\left(-\frac{\zeta(2)}{2}\right)^{s_{2}} \ldots\left(-\frac{\zeta(k)}{k}\right)^{s_{k}} .
$$

Moreover, for $l=0, . ., k$, the coefficient of $\gamma^{l}$ is of weight $k-l$.

We thereby obtain the following algebra morphism, denoted by $\gamma_{\bullet}$, for the regularization to $\gamma$ with respect to the quasi-shuffle product independently to the regularization with respect to the shuffle product ${ }^{19}$ and then by applying the tensor product of morphisms $\gamma_{\bullet} \otimes$ Id on the diagonal series, over $Y$, we get (see Annexe A)

Theorem 11. The mapping $\gamma_{\bullet}$ realizes the morphism from $(\mathbb{Q}\langle Y\rangle$, เ土 $)$ to $(\mathbb{R},$. verifying the following properties

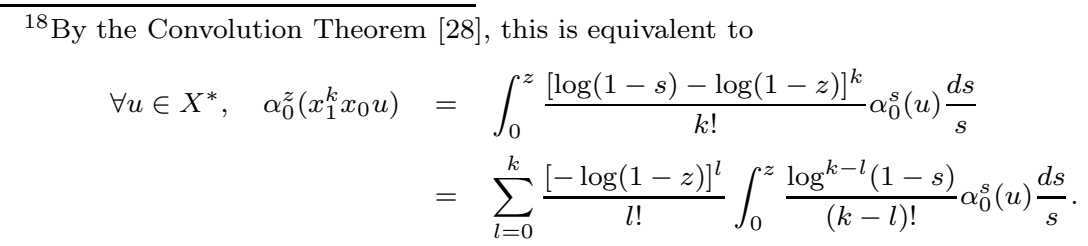

This theorem induces de facto the algebra morphism of regularization to 0 with respect to the shuffle product, as shown the Theorem 9 .

${ }^{19}$ In $[4,8,21,48]$, the authors suggest the simultaneous regularizations, with respect to the shuffle product and the quasi-shuffle product, to $T$ and then to set $T=0$. 
- for any word $u, v \in Y^{*}, \gamma_{u} \uplus v=\gamma_{u} \gamma_{v}$,

- for any convergent word $w \in Y^{*}-y_{1} Y^{*}, \gamma_{w}=\zeta(w)$

- $\gamma_{y_{1}}=\gamma$.

Then $Z_{\gamma}=e^{\gamma y_{1}} Z_{ \pm+1}$.

\subsubsection{Identities of noncommutative generating series of polyzêtas}

Corollary 6. With the notations of Definition 9, we have

$$
\begin{aligned}
Z_{\gamma}=B\left(y_{1}\right) \pi_{Y} Z_{山} & \Longleftrightarrow Z_{\uplus}=B^{\prime}\left(y_{1}\right) \pi_{Y} Z_{山}, \\
\pi_{Y} Z_{\amalg}=B^{-1}\left(x_{1}\right) Z_{\gamma} & \Longleftrightarrow Z_{\uplus}=B^{\prime-1}\left(x_{1}\right) \pi_{X} Z_{\uplus} .
\end{aligned}
$$

Roughly speaking, for the quasi-shuffle product, the regularization to $\gamma$ is "equivalent" to the regularization to 0 .

Note also that the constant $\gamma_{y_{1}}=\gamma$ is obtained as the finite part of the asymptotic expansion of $\mathrm{H}_{1}(n)$ in the comparison scale $\left\{n^{a} \log ^{b}(n)\right\}_{a \in \mathbb{Z}, b \in \mathbb{N}}$.

In the same way, since $n$ and $\mathrm{H}_{1}(n)$ are algebraically independent, as arithmetical functions (see Proposition 1), then $\left\{n^{a} \mathrm{H}_{1}^{b}(n)\right\}_{a \in \mathbb{Z}, b \in \mathbb{N}}$ constitutes a new comparison scale for asymptotic expansions.

Hence, the constants $\zeta_{\omega}\left(x_{1}\right)=0$ and $\zeta_{\boldsymbol{\omega}}\left(y_{1}\right)=0$ can be interpreted as the finite part of the asymptotic expansions of $\operatorname{Li}_{1}(z)$ and $\mathrm{H}_{1}(n)$ respectively in the comparison scales $\left\{(1-z)^{a} \log (1-z)^{b}\right\}_{a \in \mathbb{Z}, b \in \mathbb{N}}$ and $\left\{n^{a} \mathrm{H}_{1}^{b}(n)\right\}_{a \in \mathbb{Z}, b \in \mathbb{N}}$.

Definition 10 ([33]). Let $C_{1}:=\mathbb{Q} \epsilon \oplus x_{0} \mathbb{Q}\langle X\rangle x_{1}, C_{2}:=\mathbb{Q} \epsilon \oplus\left(Y-\left\{y_{1}\right\}\right) \mathbb{Q}\langle Y\rangle$.

Lemma $9([32,33])$. We get $\left(C_{1}, \omega\right) \cong\left(C_{2}\right.$, ш $)$.

Using a theorem of Radford [45] and its analogous over $Y$ (see Annexe A), we get

Proposition $8([32,33])$.

$$
\begin{aligned}
(\mathbb{Q}\langle X\rangle, \omega) \cong(\mathbb{Q}[\mathcal{L} y n X], \omega) & =C_{1}\left[x_{0}, x_{1}\right], \\
(\mathbb{Q}\langle Y\rangle, \omega) \cong(\mathbb{Q}[\mathcal{L} y n Y], \omega) & =C_{2}\left[y_{1}\right],
\end{aligned}
$$

This insures the effective way to get the finite part of the asymptotic expansions, in the comparison scales $\left\{(1-z)^{a} \log (1-z)^{b}\right\}_{a \in \mathbb{Z}, b \in \mathbb{N}}$ and $\left\{n^{a} \mathrm{H}_{1}^{b}(n)\right\}_{a \in \mathbb{Z}, b \in \mathbb{N}}$, of $\left\{\operatorname{Li}_{w}(z)\right\}_{w \in Y^{*}}$ and $\left\{\mathrm{H}_{w}(N)\right\}_{w \in Y^{*}}$ respectively.

Proposition 9 ([32, 33]). The restrictions of $\zeta_{\omega}$ and $\zeta_{\omega}$ over $\left(C_{1}, \omega\right)$ and $\left(C_{2}, \mathbf{\pm}\right)$ respectively coincide with the following surjective algebra morphism

$$
\begin{aligned}
\zeta: \begin{array}{c}
\left(C_{2}, \boldsymbol{\omega}\right) \\
\left(C_{1}, \omega\right)
\end{array} & \longrightarrow(\mathbb{R}, .) \\
y_{r_{1}} \ldots y_{r_{k}} & \longmapsto \sum_{n_{1}>\ldots>n_{k}>0} \frac{1}{n_{1}^{r_{1}} \ldots n_{k}^{r_{k}}},
\end{aligned}
$$


In Section 3.3 we will give the complete description of the kernel $\operatorname{ker} \zeta$.

With the double regularization ${ }^{20}$ to zero $[4,8,33,43]$, the Drindfel'd associator $\Phi_{K Z}$ corresponds then to $Z_{\amalg}$ (obtained with only convergent polyzêtas) as being the unique group-like element satisfying $[35,32]$

$$
\left\langle Z_{\uplus} \mid x_{0}\right\rangle=\left\langle Z_{\uplus} \mid x_{1}\right\rangle=0 \quad \text { and } \quad \forall x \in x_{0} X^{*} x_{1}, \quad\left\langle Z_{\amalg} \mid w\right\rangle=\zeta(w) .
$$

As consequence of Proposition 2, one has

Proposition 10 ([38]).

$$
\begin{aligned}
\log Z_{\amalg} & =\sum_{w \in X^{*}} \zeta_{\amalg}(w) \pi_{1}(w), \\
& =\sum_{k \geq 1} \frac{(-1)^{k-1}}{k} \sum_{u_{1}, \ldots, u_{k} \in X^{*}-\{\epsilon\}} \zeta_{\varpi}\left(u_{1} ш \ldots \sqcup u_{k}\right) u_{1} \ldots u_{k} .
\end{aligned}
$$

The associator $\Phi_{K Z}$ can be also graded in the adjoint basis of $\mathcal{U}\left(\mathcal{L} \mathcal{L}_{\mathbb{Q}}\langle X\rangle\right)$ as follows

Proposition $11([38])$. For any $l \in \mathbb{N}$ and $P \in \mathbb{C}\langle X\rangle$, let $\circ$ denotes the composite operation defined by $x_{1} x_{0}^{l} \circ P=x_{1}\left(x_{0}^{l} \uplus P\right)$. Then

$$
Z_{\uplus}=\sum_{k \geq 0} \sum_{l_{1}, \cdots, l_{k} \geq 0} \zeta_{\varpi}\left(x_{1} x_{0}^{l_{1}} \circ \cdots \circ x_{1} x_{0}^{l_{k}}\right) \prod_{i=0}^{k} \operatorname{ad}_{x_{0}}^{l_{i}} x_{1},
$$

where $\operatorname{ad}_{x_{0}}^{l} x_{1}$ is iterated Lie bracket $\operatorname{ad}_{x_{0}}^{l} x_{1}=\left[x_{0}, \operatorname{ad}_{x_{0}}^{l-1} x_{1}\right]$ and $\operatorname{ad}_{x_{0}}^{0} x_{1}=x_{1}$.

Using the following expansion [6]

$$
\operatorname{ad}_{x_{0}}^{n} x_{1}=\sum_{i=0}^{n}\left(\begin{array}{l}
i \\
n
\end{array}\right) x_{0}^{n-i} x_{1} x_{0}^{i},
$$

one deduces then, via the regularization process of Theorem 9 , the expression of the Drindfel'd associator $\Phi_{K Z}$ given by Lê and Murakami [22].

\subsection{Action of differential Galois group of polylogarithms on their asymptotic expansions}

\subsubsection{Group of associators theorem}

Let $A$ a be a commutative $\mathbb{Q}$-algebra.

Since the polyzêtas satisfy (36), then by the Friedrichs criterion we can state the following

\footnotetext{
${ }^{20}$ This double regularization is deduced from of the noncommutative generating series $Z_{\amalg}$ and $Z_{ \pm}$on the definitions 1 and 2 (see the theorems 8 and 9).
} 
Definition 11. Let $\operatorname{dm}(A)$ be the set of $\Phi \in A\langle\langle X\rangle\rangle$ such that ${ }^{21}\langle\Phi \mid \epsilon\rangle=1$, $\left\langle\Phi \mid x_{0}\right\rangle=\left\langle\Phi \mid x_{1}\right\rangle=0, \Delta_{\uplus} \Phi=\Phi \otimes \Phi$ and such that, for

$$
\Psi=B^{\prime}\left(y_{1}\right) \pi_{Y} \Phi \quad \in \quad A\langle\langle Y\rangle\rangle
$$

then $^{22} \Delta_{\amalg} \Psi=\Psi \otimes \Psi$.

Proposition 12 ([38]). If $G(z)$ and $H(z)$ are exponential solutions of (DE) then there exsists a Lie series $C \in \mathcal{L}_{i} \mathbb{C}\langle\langle X\rangle\rangle$ such that $G(z)=H(z) \exp (C)$.

Proof. Since $H(z) H(z)^{-1}=1$ then by differentiating, we have

$$
d[H(z)] H(z)^{-1}=-H(z) d\left[H(z)^{-1}\right] .
$$

Therefore if $H(z)$ is solution of Drinfel'd equation then

$$
\begin{aligned}
d\left[H(z)^{-1}\right] & =-H(z)^{-1}[d H(z)] H(z)^{-1} \\
& =-H(z)^{-1}\left[x_{0} \omega_{0}(z)+x_{1} \omega_{1}(z)\right], \\
d\left[H(z)^{-1} G(z)\right] & =H(z)^{-1}[d G(z)]+\left[d H(z)^{-1}\right] G(z) \\
& =H(z)^{-1}\left[x_{0} \omega_{0}(z)+x_{1} \omega_{1}(z)\right] G(z) \\
& -H(z)^{-1}\left[x_{0} \omega_{0}(z)+x_{1} \omega_{1}(z)\right] G(z) .
\end{aligned}
$$

By simplification, we deduce then $H(z)^{-1} G(z)$ is a constant formal power series. Since the inverse and the product of group like elements is group like then we get the expected result.

The differential $\mathcal{C}$-module $\mathcal{C}\left\{\mathrm{Li}_{w}\right\}_{w \in X^{*}}$ is the universal Picard-Vessiot extension of every linear differential equations, with coefficients in $\mathcal{C}$ and admitting $\{0,1, \infty\}$ as regular singularities. The universal differential Galois group, noted by $\operatorname{Gal}\left(\mathrm{LI}_{\mathcal{C}}\right)$, is the set of differential $\mathcal{C}$-automorphisms of $\mathcal{C}\left\{\mathrm{Li}_{w}\right\}_{w \in X^{*}}$ (i.e the automorphisms of $\mathcal{C}\left\{\mathrm{Li}_{w}\right\}_{w \in X^{*}}$ that let $\mathcal{C}$ point-wise fixed and that commute with derivation). The action of an automorphism of $\mathrm{Gal}\left(\mathrm{LI}_{\mathcal{C}}\right)$ can be determined by its action on $\mathrm{Li}_{w}$, for $w \in X^{*}$. It can be resumed as its action on the noncommutative generating series L [38] :

Let $\sigma \in \operatorname{Gal}\left(\mathrm{LI}_{\mathcal{C}}\right)$. Then

$$
\sum_{w \in X^{*}} \sigma \operatorname{Li}_{w} w=\prod_{l \in \mathcal{L} y n X}^{\searrow} e^{\sigma \operatorname{Li}_{\tilde{S}_{l}} S_{l}} .
$$

Since $d \sigma \mathrm{Li}_{x_{i}}=\sigma d \mathrm{Li}_{x_{i}}=\omega_{i}$ then by integrating the two memmbers, we obtain $\sigma \mathrm{Li}_{x_{i}}=\mathrm{Li}_{x_{i}}+c_{x_{i}}$, where $c_{x_{i}}$ is a constant of integration. More generally, for any Lyndon word $l=x_{i} l_{1}^{i_{1}} \cdots l_{k}^{i_{k}}$ with $l_{1}>\cdots>l_{k}$, one has

$$
\sigma \operatorname{Li}_{\check{S}_{l}}=\int \omega_{x_{i}} \frac{\sigma \mathrm{Li}_{\check{S}_{l_{1}}}^{i_{1}}}{i_{1} !} \cdots \frac{\sigma \mathrm{Li}_{\tilde{S}_{l_{k}}}^{i_{l_{k}}}}{i_{k} !}+c_{\check{S}_{l}}
$$

${ }^{21} \Delta_{\sqcup}$ denotes the co-product of the shufle product.

${ }^{22} \Delta_{\boldsymbol{t}}$ denotes the co-product of the quasi-shufle product. 
where $c_{\breve{S}_{l}}$ is a constant of integration. For example,

$$
\begin{aligned}
\sigma \mathrm{Li}_{x_{0} x_{1}} & =\mathrm{Li}_{x_{0} x_{1}}+c_{x_{1}} \mathrm{Li}_{x_{0}}+c_{x_{0} x_{1}}, \\
\sigma \mathrm{Li}_{x_{0}^{2} x_{1}} & =\mathrm{Li}_{x_{0}^{2} x_{1}}+\frac{c_{x_{1}}}{2} \mathrm{Li}_{x_{0}}^{2}+c_{x_{0} x_{1}} \mathrm{Li}_{x_{0}}+c_{x_{0}^{2} x_{1}}, \\
\sigma \mathrm{Li}_{x_{0} x_{1}^{2}} & =\mathrm{Li}_{x_{0} x_{1}^{2}}+c_{x_{1}} \operatorname{Li}_{x_{0} x_{1}}+\frac{c_{x_{1}}^{2}}{2} \mathrm{Li}_{x_{0}}+c_{x_{0} x_{1}^{2}} .
\end{aligned}
$$

Consequently,

$$
\sum_{w \in X^{*}} \sigma \operatorname{Li}_{w} w=\mathrm{L} e^{C_{\sigma}} \quad \text { where } \quad e^{C_{\sigma}}:=\prod_{l \in \mathcal{L} y n X}^{\searrow} e^{c_{\tilde{S}_{l}} S_{l}} .
$$

The action of $\sigma \in \operatorname{Gal}\left(\mathrm{LI}_{\mathcal{C}}\right)$ over $\left\{\mathrm{Li}_{w}\right\}_{w \in X^{*}}$ is then equivalent to the action of the Lie exponential $e^{C_{\sigma}} \in \operatorname{Gal}(D E)$ over the exponential solution L. So,

Theorem 12 ([38]). We have $\operatorname{Gal}\left(\mathrm{LI}_{\mathcal{C}}\right)=\left\{e^{C} \mid C \in \mathcal{L} i e_{\mathbb{C}}\langle\langle X\rangle\rangle\right\}$.

Typically, since $\mathrm{L}\left(z_{0}\right)^{-1}$ is group-like then $S_{z_{0} \rightsquigarrow z}=\mathrm{L}(z) \mathrm{L}\left(z_{0}\right)^{-1}$ is an other solution of (34) as already saw in (52).

Theorem 13 (Group of associators theorem). Let $\Phi \in A\langle\langle X\rangle\rangle$ and $\Psi \in A\langle\langle Y\rangle\rangle$ be group-like elements, for the co-products $\Delta_{\omega}, \Delta_{ \pm}$respectively, such that $\Psi=$ $B\left(y_{1}\right) \pi_{Y} \Phi$. There exists an unique $C \in \mathcal{L} i e_{A}\langle\langle X\rangle\rangle$ such that $\Phi=Z_{\amalg} e^{C}$ and $\Psi=B\left(y_{1}\right) \pi_{Y}\left(Z_{\sqcup} e^{C}\right)$.

Proof. If $C \in \mathcal{L i e}_{A}\langle\langle X\rangle\rangle$ then $\mathrm{L}^{\prime}=\mathrm{L} e^{C}$ is group-like, for the co-product $\Delta_{\omega}$, and $e^{C} \in \operatorname{Gal}(D E)$. Let $\mathrm{H}^{\prime}$ be the noncommutative generating series of the Taylor coefficients, belonging to the harmonic algebra, of $\left\{(1-z)^{-1}\left\langle\mathrm{~L}^{\prime} \mid w\right\rangle\right\}_{w \in Y^{*}}$. Then $\mathrm{H}^{\prime}(N)$ is also group-like, for the co-product $\Delta_{\boldsymbol{t}}$. By the asymptotic expansion of $\mathrm{L}$, we have $\mathrm{L}^{\prime}(z) \underset{\varepsilon \rightarrow 1}{\widetilde{x}} e^{-x_{1} \log (1-z)} Z_{\text {Ш }} e^{C}[36,32]$. We put then $\Phi:=Z_{\sqcup} e^{C}$ and we deduce that

$$
\frac{\mathrm{L}^{\prime}(z)}{1-z} \underset{z \rightarrow 1}{\widetilde{M}} \operatorname{Mono}(z) \Phi \quad \text { and } \quad \mathrm{H}^{\prime}(N) \underset{N \rightarrow \infty}{\widetilde{C}} \operatorname{Const}(N) \pi_{Y} \Phi,
$$

where the expressions of $\operatorname{Mono}(z)$ and $\operatorname{Const}(N)$ are given on (49) and (50) respectively. Let $\kappa_{w}$ be the constant part of $\mathrm{H}_{w}^{\prime}(N)$. Then

$$
\sum_{w \in Y^{*}} \kappa_{w} w=B\left(y_{1}\right) \pi_{Y} \Phi .
$$

We put then $\Psi:=B\left(y_{1}\right) \pi_{Y} \Phi$ (and also $\left.\Psi^{\prime}:=B^{\prime}\left(y_{1}\right) \pi_{Y} \Phi\right)$.

Corollary 7. We have

$d m(A)=\left\{Z_{\uplus} e^{C} \mid C \in \mathcal{L}_{i e}\langle\langle X\rangle\rangle \quad\right.$ and $\left.\left\langle e^{C} \mid \epsilon\right\rangle=1,\left\langle e^{C} \mid x_{0}\right\rangle=\left\langle e^{C} \mid x_{1}\right\rangle=0\right\}$.

Proof. On the one hand, $\left\langle\Phi \mid x_{0}\right\rangle=\left\langle Z_{\amalg} \mid x_{0}\right\rangle=0,\left\langle\Phi \mid x_{1}\right\rangle=\left\langle Z_{\varpi} \mid x_{1}\right\rangle=0$ and on the other, $\langle\Phi \mid \epsilon\rangle=\left\langle Z_{\amalg} \mid \epsilon\right\rangle=1$, the result follows. 
Note also that if $\mathcal{Z} \subset A$ then $\operatorname{dm}(A)$ forms a group and with the notations of Corollary 6, we obtain

Corollary 8. For any associator $\Phi=Z_{\uplus} e^{C} \in d m(A)$, let $\Psi=B\left(y_{1}\right) \pi_{Y} \Phi$ and let $\Psi^{\prime}=B^{\prime}\left(y_{1}\right) \pi_{Y} \Phi$. Then

$$
\Psi=B\left(y_{1}\right) \pi_{Y} \Phi \quad \Longleftrightarrow \quad \Psi^{\prime}=B^{\prime}\left(y_{1}\right) \pi_{Y} \Phi .
$$

Proof. Since $\Psi$ is group like and since $\left\langle\Phi \mid x_{1}\right\rangle=\left\langle\Psi^{\prime} \mid y_{1}\right\rangle=0$ and $\left\langle\Psi \mid y_{1}\right\rangle=\gamma$ then, using the factorization by Lyndon words, we get the expected result.

Lemma 10. Let $\Phi=Z_{\varpi} e^{C} \in d m(A)$ and let $\Psi=B\left(y_{1}\right) \pi_{Y}\left(Z_{\amalg} e^{C}\right)$. The local coordinates (of second kind) of $\Phi$ (resp. $\Psi$ ) are polynomials on $\left\{\zeta_{\omega}\left(\check{S}_{l}\right)\right\}_{l \in \mathcal{L} y n X}$ (resp. $\left.\left\{\zeta_{\omega_{+}}\left(\check{\Sigma}_{l}\right)\right\}_{l \in \mathcal{L} y n Y}\right)$ of $\mathcal{Z}$ (resp. $\left.\mathcal{Z}^{\prime}\right)$. While $C$ describes $\mathcal{L} i e_{A}\langle\langle X\rangle)$, these coordinates describe $A\left[\left\{\zeta_{\omega}\left(\check{S}_{l}\right)\right\}_{l \in \mathcal{L} y n X}\right]$ (resp. $A\left[\left\{\zeta_{\omega}\left(\check{\Sigma}_{l}\right)\right\}_{l \in \mathcal{L} y n Y}\right]$ ).

Proof. Let $\Phi \in d m(A)$. By Corollary 7 , there exists $P \in \mathcal{L} i_{A}\langle\langle X\rangle\rangle$ verifying $\left\langle e^{P} \mid \epsilon\right\rangle=1,\left\langle e^{P} \mid x_{0}\right\rangle=\left\langle e^{P} \mid x_{1}\right\rangle=0$ such that $\Phi=Z_{\omega} e^{P}$. Using the factorization forms by Lyndon words, we get

$$
\prod_{l \in \mathcal{L} y n X-X}^{\searrow} e^{\phi\left(\check{S}_{l}\right) S_{l}}=\left(\prod_{l \in \mathcal{L} y n X-X}^{\searrow} e^{\zeta\left(\check{S}_{l}\right) S_{l}}\right)\left(\prod_{l \in \mathcal{L} \text { yn } X-X}^{\searrow} e^{p_{\check{S}_{l}} S_{l}}\right) .
$$

Expanding the Hausdorff product and identifying the local coordinates in the PBW-Lyndon basis there exists $I_{l} \subset\{\lambda \in \mathcal{L} y n X-X$ s.t. $|\lambda| \leq|l|\}$, for $l \in$ $\mathcal{L} y n X-X$, and the coefficients $\left\{p_{\check{S}_{u}}^{\prime}\right\}_{u \in I_{l}}$ belonging to $A$ such that

$$
\phi\left(\check{S}_{l}\right)=\sum_{u \in I_{l}} p_{\check{S}_{u}}^{\prime} \zeta\left(\check{S}_{u}\right) .
$$

This belongs to $A\left[\left\{\zeta\left(\breve{S}_{l}\right)\right\}_{l \in \mathcal{L} y n X-X]}\right]$ and holds for any $P \in \mathcal{L i e}_{A}\langle\langle X\rangle$.

With the notations of Definition 9 and by Corollary 8 , we get in particular

Lemma 11. For any $\Phi \in d m(A)$, by identifying the local coordinates (of second kind) on two members of the identities $\Psi=B\left(y_{1}\right) \pi_{Y} \Phi$, or equivalently of $\Psi^{\prime}=$ $B^{\prime}\left(y_{1}\right) \pi_{Y} \Phi$, we get polynomial relations, of coefficients in $A$, among generators of the A-algebra of convergent polyzêtas.

Therefore,

Theorem 14. While $\Phi$ describes $d m(A)$, the identities $\Psi=B\left(y_{1}\right) \pi_{Y} \Phi$ describe the ideal of polynomial relations, of coefficients in A, among generators of the A-algebra of convergent polyzêtas. Moreover, if the Euler constant, $\gamma$, does not belong to $A$ then these relations are algebraically independent on $\gamma$.

Simplyfied computations on Section 3.3 is an example of such identities. Some consequences of Theorem 14 will be drawn in Section 4.2. 


\subsubsection{Concatenation of Chen generating series}

As an example of the action of the differential Galois group of polylogarithms on their asymptotic expansions, we are interrested on the action of their monodromy group which is contained in $\operatorname{Gal}(D E)$.

The monodromies at 0 and 1 of $\mathrm{L}$ are given respectively by $[35,32]$

$$
\begin{gathered}
\mathcal{M}_{0} \mathrm{~L}=\mathrm{L} e^{2 \mathrm{i} \pi \mathfrak{m}_{0}} \quad \text { and } \quad \mathcal{M}_{1} \mathrm{~L}=\mathrm{L} Z_{\amalg}^{-1} e^{-2 \mathrm{i} \pi x_{1}} Z_{山}=\mathrm{L} e^{2 \mathrm{i} \pi \mathfrak{m}_{1}} \\
\text { where } \quad \mathfrak{m}_{0}=x_{0} \quad \text { and } \quad \mathfrak{m}_{1}=\prod_{l \in \mathcal{L} y n X-X}^{>} e^{-\zeta\left(\check{S}_{l}\right) \operatorname{ad}_{S_{l}}}\left(-x_{1}\right) .
\end{gathered}
$$

- If $C=2 \mathrm{i} \pi \mathfrak{m}_{0}$ then

$$
\begin{aligned}
\Phi & =Z_{\uplus} e^{2 \mathrm{i} \pi x_{0}} \\
\Psi & =\exp \left(\gamma y_{1}-\sum_{k \geq 2} \zeta(k) \frac{\left(-y_{1}\right)^{k}}{k}\right) \pi_{Y} Z_{\varpi} \\
& =Z_{\amalg} .
\end{aligned}
$$

The monodromy at 0 consists in the multiplication on the right of $Z_{\amalg}$ by $e^{2 \mathrm{i} \pi x_{0}}$ and does not modify $Z_{ \pm+}$.

- If $C=2 \mathrm{i} \pi \mathfrak{m}_{1}$ then

$$
\begin{aligned}
\Phi & =e^{-2 \mathrm{i} \pi x_{1}} Z_{\amalg}, \\
\Psi & =\exp \left((\underbrace{\gamma-2 \mathrm{i} \pi}_{T:=}) y_{1}-\sum_{k \geq 2} \zeta(k) \frac{\left(-y_{1}\right)^{k}}{k}\right) \pi_{Y} Z_{\amalg} \\
& =e^{-2 \mathrm{i} \pi y_{1}} Z_{\amalg} .
\end{aligned}
$$

The monodromy at 1 consists in the multiplication on left of $Z_{\varpi}$ and of $Z_{\uplus}$ by $e^{-2 \mathrm{i} \pi x_{1}}$ and $e^{-2 \mathrm{i} \pi y_{1}}$ respectively.

Remark 1. 1. The monodromies around singularities of $\mathrm{L}$ could not allow, in this case, neither to introduce the factor $e^{\gamma x_{1}}$ on the left of $Z_{w}$ nor to eliminate the left factor $e^{\gamma y_{1}}$ in $Z_{\gamma}$ (by putting ${ }^{23} T=0$, for example).

2. By Proposition 5, we already saw that $Z_{\varpi}$ is the concatenation of Chen generating series [10] $e^{x_{0} \log \varepsilon}$ and then $S_{\varepsilon_{\rightsquigarrow 1-\varepsilon}}$ and finally, $e^{x_{1} \log \varepsilon}$ :

$$
Z_{山} \underset{\varepsilon \rightarrow 0^{+}}{\widetilde{x^{\prime}}} e^{x_{1} \log \varepsilon} S_{\varepsilon \rightsquigarrow 1-\varepsilon} e^{x_{0} \log \varepsilon} .
$$

From (72) and (75), the action of the monodromy group gives

$$
e^{x_{1} 2 k_{1} \mathrm{i} \pi} Z_{\sqcup} e^{x_{0} 2 k_{0} \mathrm{i} \pi} \underset{\varepsilon \rightarrow 0^{+}}{\widetilde{x}} e^{x_{1}\left(\log \varepsilon+2 k_{1} \mathrm{i} \pi\right)} S_{\varepsilon \rightsquigarrow 1-\varepsilon} e^{x_{0}\left(\log \varepsilon+2 k_{0} \mathrm{i} \pi\right)}(79)
$$

${ }^{23}$ Why? 
as being the concatenation of the Chen generating series $e^{x_{0}\left(\log \varepsilon+2 k_{0} \mathrm{i} \pi\right)}$ (along circular path turning $k_{0}$ times around 0 ), then the Chen generating series $S_{\varepsilon \rightsquigarrow 1-\varepsilon}$ and finally, the Chen generating series $e^{x_{1}\left(\log \varepsilon+2 k_{1} \mathrm{i} \pi\right)}$ (along circular path turning $k_{1}$ times around 1$)$.

3. More generally, by Corollary 7, the action of the Galois differential group of polylogarithms states, for any Lie series $C$, the associator $\Phi=Z_{\amalg} e^{C}$ is the concatenation of some Chen generating series $e^{C}$ and $e^{x_{0} \log \varepsilon}$ and then the Chen generating series $S_{\varepsilon \rightsquigarrow 1-\varepsilon}$ and finally, $e^{x_{1} \log \varepsilon}$ :

$$
\Phi \underset{\varepsilon \rightarrow 0^{+}}{\widetilde{x^{\prime}}} e^{x_{1} \log \varepsilon} S_{\varepsilon \rightsquigarrow 1-\varepsilon} e^{x_{0} \log \varepsilon} e^{C} .
$$

By construction (see Theorem 13) the associator $\Phi$ is then the noncommutative generating series of the finite parts of the coefficients of the Chen generating series $S_{z_{0} \rightsquigarrow 1-z_{0}} e^{C}$, for $z_{0}=\varepsilon \rightarrow 0^{+}$. Hence,

Corollary 9. Let $\Phi \in d m(A)$. For any differential produced formal power series $S$ over $X$, there exists ${ }^{24}$ a differential representation $(\mathcal{A}, f)$ such that :

$$
\langle\Phi \| S\rangle=\sum_{w \in X^{*}}\langle\Phi \mid w\rangle \mathcal{A}(w) \circ f_{\left.\right|_{0}}=\prod_{l \in \mathcal{L} y n X-X}^{\searrow} e^{\left\langle\Phi \mid \check{S}_{l}\right\rangle \mathcal{A}\left(S_{l}\right)} \circ f_{\left.\right|_{0}} .
$$

\subsection{Algebraic combinatorial studies of polynomial relation among polyzêta via a group of associators}

Here, $\bar{Y}=\left\{y_{1}\right\} \cup\left\{\bar{y}_{k}\right\}_{k \geq 2}$. With the factorization of the monoids $X^{*}$ and $\bar{Y}^{*}$ by Lyndon words, let $\{\hat{l}\}_{l \in \mathcal{L} y n X}$ and $\{\hat{l}\}_{l \in \mathcal{L} y n \bar{Y}}$ be the dual of the Lyndon basis over $X$ and $\bar{Y}$.

\subsubsection{Preliminary study}

As in Definition 10, let

$$
A_{1}=A \epsilon \oplus x_{0} A\langle X\rangle x_{1} \quad \text { and } \quad A_{2}=A \epsilon \oplus\left(\bar{Y}-\left\{y_{1}\right\}\right) A\langle\bar{Y}\rangle .
$$

For $\Phi \in d m(A)$, let $\Psi=B^{\prime}\left(y_{1}\right) \pi_{\bar{Y}} \Phi$. Let us introduce two algebra morphisms

$$
\begin{aligned}
& \phi:\left(A_{1}, ш\right) \quad \longrightarrow \quad A, \quad \psi:\left(A_{2}, \text { ш }\right) \rightarrow \quad \rightarrow \quad A, \\
& u \quad \longmapsto\langle\Phi \mid u\rangle, \quad v \quad \longmapsto\langle\Psi \mid v\rangle,
\end{aligned}
$$

verifying respectively $\phi(\epsilon)=1, \phi\left(x_{0}\right)=\phi\left(x_{1}\right)=0$ and $\psi(\epsilon)=1, \psi\left(y_{1}\right)=0$.

Lemma 12. For any $\Phi \in d m(A)$, let $\Psi=B^{\prime}\left(y_{1}\right) \pi_{Y} \Phi$. Then

$$
\begin{aligned}
\forall w \in \bar{Y}^{*}-y_{1} \bar{Y}^{*}, & \psi(w) & =\phi\left(\pi_{X} w\right), \\
\text { or equivalently, } \quad \forall w \in x_{0} X^{*} x_{1}, & \phi(w) & =\psi\left(\pi_{\bar{Y}} w\right) .
\end{aligned}
$$

${ }^{24}$ See Corollary 22 of Annexe B. 
Lemma 13. We have

$\Phi=\sum_{u \in X^{*}} \phi(u) u=\prod_{l \in \mathcal{L} y n X-X}^{\searrow} e^{\phi(l) \hat{l}} \quad$ and $\quad \Psi=\sum_{v \in \bar{Y}^{*}} \psi(u) u=\prod_{l \in \mathcal{L} y n \bar{Y}-\left\{y_{1}\right\}}^{\searrow} e^{\psi(l) \hat{l}}$.

With the notations in Lemma 13, we can state the following

Definition 12. We put

$$
\mathcal{R}:=\bigcap_{\Phi \in d m(A)} \operatorname{ker} \phi \quad\left(\text { resp. } \bigcap_{\substack{\Psi=B^{\prime}\left(y_{1}\right) \pi \bar{Y}^{\Phi} \\ \Phi \in d m(A)}} \operatorname{ker} \psi\right) .
$$

Lemma 14. For any $\Phi \in d m(A)$, let $\Psi=B^{\prime}\left(y_{1}\right) \pi_{\bar{Y}} \Phi$. Let $Q \in \mathbb{Q}[\mathcal{L} y n X]$ (resp. $\mathbb{Q}[\mathcal{L} y n \bar{Y}])$. Then

$$
\langle Q \| \Phi\rangle=0 \Longleftrightarrow Q \in \operatorname{ker} \phi \quad(\text { resp. } \quad\langle Q \| \Psi\rangle=0 \Longleftrightarrow Q \in \operatorname{ker} \psi) .
$$

Or equivalently (see Definition 7),

$$
Q \in \mathcal{R} \quad \Longleftrightarrow \quad Q \text { is indiscernable over } \operatorname{dm}(A) .
$$

Let $\Phi_{1}, \Phi_{2} \in d m(A)$. By Corollary 7 , for $i=1$ or 2 , there exists an unique $P_{i} \in \mathcal{L} i e_{A}\langle\langle X\rangle\rangle$ such that $e^{-P_{i}}$ is well defined and

$$
\Phi_{i}=Z_{\varpi} e^{P_{i}}, \quad \text { or equivalently, } \quad Z_{山}=\Phi_{1} e^{-P_{1}}=\Phi_{2} e^{-P_{2}} .
$$

Then, we get $\Phi_{1}=\Phi_{2} e^{P_{1}-P_{2}}$ and $\Phi_{2}=\Phi_{1} e^{P_{2}-P_{1}}$. By Lemma 10 , it follows

Lemma 15. Let $\Phi_{1}$ and $\Phi_{2} \in d m(A)$. For any convergent Lyndon word, $l$, there exists a finite set $I_{l} \subset\{\lambda \in \mathcal{L} y n X-X$ s.t. $|\lambda| \leq|l|\}$ and the coefficients $\left\{p_{i, u}^{\prime}\right\}_{u \in I_{l}}$ and $\left\{p_{i, u}^{\prime \prime}\right\}_{u \in I_{l}}$, for $i=1$ or 2 , belonging to $A$ such that

$$
\phi_{i}(l)=\sum_{u \in I_{l}} p_{i, u}^{\prime} \zeta(u), \quad \text { or equivalently, } \quad \zeta(l)=\sum_{u \in I_{l}} p_{i, u}^{\prime \prime} \phi_{i}(u) .
$$

There also exists the coefficients $\left\{p_{u}^{\prime}\right\}_{u \in I_{l}}$ and $\left\{p_{u}^{\prime \prime}\right\}_{u \in I_{l}}$ belonging to $A$ such that

$$
\phi_{1}(l)=\sum_{u \in I_{l}} p_{u}^{\prime} \phi_{2}(u), \quad \text { or equivalently, } \quad \phi_{2}(l)=\sum_{u \in I_{l}} p_{u}^{\prime \prime} \phi_{1}(u) .
$$

Therefore, the $\left\{\phi_{i}(l)\right\}_{l \in \mathcal{L} y n X-X}$ (resp. $\left\{\psi_{i}(l)\right\}_{l \in \mathcal{L} y n \bar{Y}-\left\{y_{1}\right\}}$ ), for $i=1$ or 2 , are also generators of the $A$-algebra generated by convergent polyzêtas.

\subsubsection{Description of polynomial relations among coefficients of asso- ciator and irreducible polyzêtas}

Since the identities of Corollary 8 (see also Corollary 6) hold for any pair of bases, in duality, compatible with factorization of the monoid $X^{*}\left(\operatorname{resp} . \bar{Y}^{*}\right)$ then, by Corollary 8 , one gets 
Theorem 15. For any $\Phi \in d m(A)$, let $\Psi=B^{\prime}\left(y_{1}\right) \pi_{\bar{Y}} \Phi$. We have

$$
\prod_{l \in \mathcal{L} y n \bar{Y}-y_{1}}^{\searrow} e^{\psi(l) \hat{l}}=\exp \left(\sum_{k \geq 2} \zeta(k) \frac{\left(-y_{1}\right)^{k}}{k}\right) \pi_{\bar{Y}} \prod_{l \in \mathcal{L} y n X-X}^{\searrow} e^{\phi(l) \hat{l}} .
$$

If $\Phi=Z_{\sqcup}$ and $\Psi=Z_{\text {山 }}$ then, for $\ell \in \mathcal{L} y n X-X$ (resp. $\mathcal{L} y n \bar{Y}-y_{1}$ ), one has $\zeta(l)=\phi(l)$ (resp. $\psi(l))$. Hence, one ontains (see also Corollary 6 )

Theorem 16 (Bis repetita).

$$
\prod_{l \in \mathcal{L} y n \bar{Y}-y_{1}}^{\searrow} e^{\zeta(l) \hat{l}}=\exp \left(\sum_{k \geq 2} \zeta(k) \frac{\left(-y_{1}\right)^{k}}{k}\right) \pi_{\bar{Y}} \prod_{l \in \mathcal{L} y n X-X}^{\searrow} e^{\zeta(l) \hat{l}} .
$$

Corollary 10. For any $\ell \in \mathcal{L} y n \bar{Y}-y_{1}($ resp. $\mathcal{L} y n X-X)$, let $P_{\ell} \in \mathcal{U}\left(\mathcal{L} i e_{\mathbb{Q}}\langle X\rangle\right)$ (resp. $\mathcal{U}\left(\mathcal{L} i e_{\mathbb{Q}}\langle\bar{Y}\rangle\right)$ ) be the decomposition of the polynomial $\pi_{X} \hat{\ell} \in \mathbb{Q}\langle X\rangle$ (resp. $\left.\pi_{\bar{Y}} \hat{\ell} \in \mathbb{Q}\langle\bar{Y}\rangle\right)$ in the PBW basis, induced by $\{\hat{l}\}_{l \in \mathcal{L} y n X}$ (resp. $\{\hat{l}\}_{l \in \mathcal{L} y n \bar{Y}}$ ), and let $\check{P}_{\ell} \in \mathbb{Q}[\mathcal{L} y n X-X]$ (resp. $\left.\mathbb{Q}\left[\mathcal{L} y n \bar{Y}-y_{1}\right]\right)$ be its dual. Then one obtains

$$
\pi_{X} \ell-\check{P}_{\ell} \in \operatorname{ker} \phi \quad\left(\text { resp. } \quad \pi_{\bar{Y}} \ell-\check{P}_{\ell} \in \operatorname{ker} \psi\right)
$$

In particular, for $\phi=\zeta$ (resp. $\psi=\zeta)$ then one also obtains

$$
\pi_{X} \ell-\check{P}_{\ell} \in \operatorname{ker} \zeta \quad\left(\text { resp. } \quad \pi_{\bar{Y}} \ell-\check{P}_{\ell} \in \operatorname{ker} \zeta\right) .
$$

Moreover, for any $\ell \in \mathcal{L} y n \bar{Y}-y_{1}$ (resp. $\left.\mathcal{L} y n X-X\right)$, the homogenous polynomial $\pi_{X} \ell-\check{P}_{\ell} \in \mathbb{Q}\langle X\rangle$ (resp. $\left.\mathbb{Q}\langle\bar{Y}\rangle\right)$ is of degree equal $|\ell| \geq 2$.

Proof. Since

$$
\ell \in \mathcal{L} y n \bar{Y} \quad \Longleftrightarrow \quad \pi_{X} \ell \in \mathcal{L} y n X-\left\{x_{0}\right\}
$$

then identifying the local coordinates (of second kind) on the two members of each identity in Theorem 15, one obtains

$$
\begin{aligned}
\forall \ell \in \mathcal{L} y n \bar{Y}-y_{1} \subset Y^{*}-y_{1} Y^{*}, & & \psi(\ell)=\phi\left(\check{P}_{\ell}\right), \\
\text { resp. } \forall \ell \in \mathcal{L} y n X-X \subset x_{0} X^{*} x_{1}, & \phi(\ell) & \left.=\psi\left(\check{P}_{\ell}\right)\right) .
\end{aligned}
$$

By Lemma 12, we get the expected result.

With the notations of Corollary 10, we get the following

Definition 13. Let $Q_{\ell}$ be the decomposition of the proper polynomial $\pi_{\bar{Y}} \ell-\check{P}_{\ell}$ (resp. $\left.\pi_{X} \ell-\check{P}_{\ell}\right)$ in $\mathcal{L} y n \bar{Y}($ resp. $\mathcal{L} y n X)$. Let

$$
\begin{aligned}
& \mathcal{R}_{\bar{Y}}:=\left\{Q_{\ell}\right\}_{\ell \in \mathcal{L} y n \bar{Y}-y_{1}} \quad \text { and } \quad \mathcal{R}_{X}:=\left\{Q_{\ell}\right\}_{\ell \in \mathcal{L} y n X-X}, \\
& \mathcal{L}_{i r r} \bar{Y}:=\left\{\ell \in \mathcal{L} y n \bar{Y}-y_{1} \mid Q_{\ell}=0\right\} \quad \text { and } \quad \mathcal{L}_{\text {irr }} X:=\left\{\ell \in \mathcal{L} y n X-X \mid Q_{\ell}=0\right\} .
\end{aligned}
$$

It follows that 
Lemma 16. We have

$$
\begin{aligned}
\left(\mathbb{Q}\left[\mathcal{L} y n \bar{Y}-y_{1}\right], \uplus\right) & =\left(\mathcal{R}_{\bar{Y}}, \uplus\right) \oplus\left(\mathbb{Q}\left[\mathcal{L}_{i r r} \bar{Y}\right], \uplus\right), \\
(\mathbb{Q}[\mathcal{L} y n X-X], \omega) & =\left(\mathcal{R}_{X}, \omega\right) \oplus\left(\mathbb{Q}\left[\mathcal{L}_{i r r} X\right], \omega\right) .
\end{aligned}
$$

Then we can state the following

Definition 14. Any word $w$ is said to be irreducible if and only if $w$ belongs to $\mathcal{L}_{\text {irr }} \bar{Y}$ (resp. $\left.\mathcal{L}_{i r r} X\right)$. In this case, the polyzêta $\zeta(w)$ est said to be $\mathbb{Q}$-irreducible.

For any $P \in \mathbb{Q}\left[\mathcal{L}_{i r r} X\right]$, there exists ${ }^{25}$ a differential representation $(\mathcal{A}, f)$ such that $P$ can be finitely factorized (see also Corollary 9 ) :

$$
P=\sigma f_{\left.\right|_{0}}=\sum_{w \in X_{\text {irr }}^{*}} \mathcal{A}(w) \circ f w=\prod_{\ell \in \mathcal{L}_{i r r} X, \text { finite }}^{\searrow} e^{\mathcal{A}(\hat{\ell}) \ell} \circ f,
$$

where $X_{i r r}^{*}$ denotes the set of words obtaining by shuffling on $\mathcal{L}_{i r r} X$.

Lemma 17. Any proper polynomial $P \in\left(\mathbb{Q}\left[\mathcal{L}_{\text {irr }} X\right], \omega\right)\left(\right.$ resp. $\left.\left(\mathbb{Q}\left[\mathcal{L}_{i r r} \bar{Y}\right], \omega\right)\right)$ is indiscernable over Chen generating series $\left\{e^{t x}\right\}_{x \in X}^{t \in \mathbb{C}}$ :

$$
\left\langle P \| e^{t x_{0}}\right\rangle=\left\langle P \| e^{t x_{1}}\right\rangle=0 \quad\left(\text { resp. } \quad\left\langle P \| e^{t y_{1}}\right\rangle=0\right) .
$$

Proof. By construction, $x_{0}$ and $x_{1} \notin \mathcal{L}_{i r r} X$ (resp. $\left.y_{1} \notin \mathcal{L}_{i r r} X\right)$. For any $n>1, x_{0}^{n}$ and $x_{1}^{n}$ (resp. $y_{1}^{n}$ ) are not Lyndon words then they do not belong to $\mathcal{L}_{\text {irr }} X$ (resp. $\left.\mathcal{L}_{\text {irr }} X\right)$. Therefore, for any $n \geq 0$, one has $\left\langle P \mid x_{0}^{n}\right\rangle=\left\langle P \mid x_{1}^{n}\right\rangle=0$ (resp. $\left\langle P \mid y_{1}^{n}\right\rangle=0$ ). Using the expansion of the exponential, we find the expected result.

Lemma 18. Let $\Phi \in d m(A)$ and let $t \in \mathbb{C}, x \in X$. For any proper polynomial $P \in\left(\mathbb{Q}\left[\mathcal{L}_{\text {irr }} X\right], \omega\right)$, if $\langle P \| \Phi\rangle=0$ then $\left\langle P \| \Phi e^{t x}\right\rangle=0$ and $\left\langle P \| e^{t x} \Phi\right\rangle=0$.

Proof. Since $P \in\left(\mathbb{Q}\left[\mathcal{L}_{i r r} X\right], w\right)$ and $P$ is proper then, by Lemma 17 , for any $t \in \mathbb{C}$ and for any $x \in X$, we have $\left\langle P \| e^{t x}\right\rangle=0$ and then $\left\langle P \| \Phi e^{t x}\right\rangle=0$.

Since $\operatorname{supp}(P) \subset x_{0} X^{*} x_{1}$ then $\left\langle P \| e^{t x_{0}} \Phi\right\rangle=\left\langle P \triangleright e^{t x_{0}} \| \Phi\right\rangle=0$.

Next, for $\Phi \in d m(A)$, there exists $e^{C}$ such that $e^{t x_{1}} \Phi=e^{t x_{1}} Z_{\uplus} e^{C}$ and, by Proposition 5, we get

$$
e^{t x_{1}} \Phi \underset{\varepsilon \rightarrow 0^{+}}{\widetilde{x^{\prime}}} e^{x_{1}(t+\log \varepsilon)} S_{\varepsilon \rightsquigarrow 1-\varepsilon} e^{x_{0} \log \varepsilon} e^{C} .
$$

Hence, there exists a Chen generating series $C_{z \rightsquigarrow 1-z_{0}}$ and $S_{z_{0} \rightsquigarrow 1-z_{0}}$ such that we get the following asymptotic behaviour (see Section 3.2.2)

$$
e^{t x_{1}} \Phi \underset{\varepsilon \rightarrow 0^{+}}{\sim} C_{z \rightsquigarrow 1-z_{0}} S_{z_{0} \rightsquigarrow z} e^{C}
$$

and the following concatenation holds [10] (see Formula (53))

$$
\begin{aligned}
C_{z \rightsquigarrow 1-z_{0}} S_{z_{0} \rightsquigarrow z} & =S_{z_{0} \rightsquigarrow 1-z_{0}}, \\
\Longleftrightarrow \quad C_{z \rightsquigarrow 1-z_{0}} S_{z_{0} \rightsquigarrow z} e^{C} & =S_{z_{0} \rightsquigarrow 1-z_{0}} e^{C} .
\end{aligned}
$$

${ }^{25}$ See Corollary 22 of Annexe B. 
Since $P \in \mathbb{Q}\left[\mathcal{L}_{i r r} X\right]$ then by (84), applying $\left\langle\sigma f_{\left.\right|_{0}} \| \bullet\right\rangle$ to the two sides of the previous equality, one has

$$
\left\langle\sigma f_{\left.\right|_{0}} \| C_{z \rightsquigarrow 1-z_{0}} S_{z_{0} \rightsquigarrow z} e^{C}\right\rangle=\left\langle\sigma f_{\left.\right|_{0}} \| S_{z_{0} \rightsquigarrow 1-z_{0}} e^{C}\right\rangle .
$$

Thus, for $z_{0}=\varepsilon \rightarrow 0^{+}$, one obtains

$$
\left\langle\sigma f_{\left.\right|_{0}} \| e^{t x_{1}} \Phi\right\rangle \underset{\varepsilon \rightarrow 0^{+}}{\widetilde{2}}\left\langle\sigma f_{\left.\right|_{0}} \| \Phi\right\rangle .
$$

Since $\left\langle\sigma f_{\left.\right|_{0}} \| \Phi\right\rangle=\langle P \| \Phi\rangle=0$ then we get the expected result.

Lemma 19. For any $\Phi \in d m(A)$, let $\Psi=B^{\prime}\left(y_{1}\right) \pi_{\bar{Y}} \Phi$. We have $\mathcal{R}_{\bar{Y}} \subseteq \operatorname{ker} \psi$ and $\mathcal{R}_{X} \subseteq \operatorname{ker} \phi$. In particular, $\mathcal{R}_{\bar{Y}} \subseteq \operatorname{ker} \zeta$ and $\mathcal{R}_{X} \subseteq \operatorname{ker} \zeta$.

Proposition 13. We have $\mathcal{R}_{X} \subseteq \mathcal{R}$ (resp. $\left.\mathcal{R}_{\bar{Y}} \subseteq \mathcal{R}\right)$.

Proposition 14. For any proper polynomial $Q \in \mathbb{Q}\left[\mathcal{L}_{i r r} X\right]$ (resp. $\mathbb{Q}\left[\mathcal{L}_{i r r} \bar{Y}\right]$ ),

$$
Q \in \mathcal{R} \quad \Longleftrightarrow \quad Q=0 .
$$

Proof. If $Q=0$ then since, for $\Phi \in d m(A), \phi$ is an algebra homorphism then $\phi(Q)=0$. Hence, $Q \in \operatorname{ker} \phi$ and then $Q \in \mathcal{R}$.

Conversely, if $Q \in \mathcal{R}$ then, for $\Phi \in d m(A)$, we get $\langle Q \| \Phi\rangle=0$. That means $Q$ is indiscernable over $d m(A)$. Let $\mathcal{H}$ be the monoid generated by $d m(A)$ and by the Chen generating series $\left\{e^{t x}\right\}_{x \in X}^{t \in \mathbb{C}}$. By Lemma 25, $Q$ is continuous over $\mathcal{H}$ and by Lemma 18 , it is indiscernable over $\mathcal{H}$. By Proposition 7 , the expected result follows.

Therefore, by the propositions 13 and 14, we obtain

Theorem 17. We have $\mathcal{R}=\mathcal{R}_{X}\left(\right.$ resp. $\left.\mathcal{R}_{\bar{Y}}\right)$.

Proposition 15. For any $\Phi \in d m(A)$, let $\Psi=B^{\prime}\left(y_{1}\right) \pi_{\bar{Y}} \Phi$. Let $Q \in\left(\mathbb{Q}\left[\mathcal{L}_{\text {irr }} X\right]\right.$, $)$ (resp. $\left(\mathbb{Q}\left[\mathcal{L}_{i r r} \bar{Y}\right], \pm\right)$ ) such that $\langle\Phi \| Q\rangle=0$ (resp. $\langle\Psi \| Q\rangle=0$ ). Then $Q=0$.

Proof. Let $\mathcal{H}$ defined as being the monoid generated by $\Phi$ and by Chen generating series $\left\{e^{t x}\right\}_{x \in X}^{t \in \mathbb{C}}$. By assumption, $\langle\Phi \| Q\rangle=0$ and by Lemma 18, $Q$ is then indiscernable over $\mathcal{H}$. Finally, by Proposition 7, it follows that $Q=0$.

Proposition 16. For any $\Phi \in d m(A)$, let $\Psi=B^{\prime}\left(y_{1}\right) \pi_{\bar{Y}} \Phi$. We get $\operatorname{ker} \phi=\mathcal{R}_{X}$ $\left(\right.$ resp. $\left.\operatorname{ker} \psi=\mathcal{R}_{\bar{Y}}\right)$. In particular, $\operatorname{ker} \zeta=\mathcal{R}_{X}\left(\right.$ resp. $\left.\operatorname{ker} \zeta=\mathcal{R}_{\bar{Y}}\right)$.

Proof. By Lemma $19, \mathcal{R}_{X}$ and $\mathcal{R}_{\bar{Y}}$ are included in $\operatorname{ker} \phi$ and $\operatorname{ker} \psi$ respectively. Conversely, two cases can occur (see Lemma 16) :

1. Case $Q \notin \mathbb{Q}\left[\mathcal{L}_{i r r} X\right]$ (resp. $\mathbb{Q}\left[\mathcal{L}_{i r r} \bar{Y}\right]$ ). By Lemma $16, Q \equiv_{\mathcal{R}_{X}} Q_{1}$ (resp. $\left.Q \equiv_{\mathcal{R}_{\bar{Y}}} Q_{1}\right)$ such that $Q_{1} \in \mathbb{Q}\left[\mathcal{L}_{i r r} X\right]$ (resp. $\left.\mathbb{Q}\left[\mathcal{L}_{\text {irr }} \bar{Y}\right]\right)$ and $\phi\left(Q_{1}\right)=0$ (resp. $\psi\left(Q_{1}\right)=0$ ). This case is then reduced to the following

2. Case $Q \in \mathbb{Q}\left[\mathcal{L}_{i r r} X\right]$ (resp. $\mathbb{Q}\left[\mathcal{L}_{i r r} \bar{Y}\right]$ ). Using Proposition 15 , we have $Q \equiv_{\mathcal{R}_{X}} 0$ (resp. $\left.Q \equiv_{\mathcal{R}_{\bar{Y}}} 0\right)$. 
Then, $\mathcal{R}_{X}\left(\right.$ resp. $\left.\mathcal{R}_{\bar{Y}}\right)$ contains $\operatorname{ker} \phi($ resp. $\operatorname{ker} \psi)$.

For any $Q \in\left(\mathbb{Q}\left[\mathcal{L}_{i r r} X\right], \omega\right)\left(\operatorname{resp} .\left(\mathbb{Q}\left[\mathcal{L}_{i r r} \bar{Y}\right], \omega\right)\right), \zeta(Q)$ is then a polynomial on $A$-irreducible polyzêtas (see Definition 14). Moreover,

Proposition 17. The $\mathbb{Q}$-algebra $\mathcal{Z}$ is generated by the family of $A$-irreducible polyzêtas $\{\zeta(\ell)\}_{\ell \in \mathcal{L}_{i r r} \bar{Y}}\left(\right.$ resp. $\left.\{\zeta(\ell)\}_{\ell \in \mathcal{L}_{i r r} X}\right)$.

Proof. By Radford's theorem [45], one just needs to prove for Lyndon words :

Let $\ell \in \mathcal{L} y n \bar{Y}-y_{1}$. If $\pi_{X} \ell=\breve{P}_{\ell}$ then the result follows else one has $\pi_{X} \ell-\check{P}_{\ell} \in$ $\operatorname{ker} \zeta$. Hence, $\zeta(\ell)=\zeta\left(\check{P}_{\ell}\right)$.

Since $\check{P}_{\ell} \in \mathbb{Q}[\mathcal{L} y n X-X]$ then $\check{P}_{\ell}$ is polynomial on Lyndon words, over $X$, of degree less or equal $|\ell|$. For each Lyndon word does appear in this decomposition of $\check{P}_{\ell}$, after applying $\pi_{\bar{Y}}$, one uses the same recurcive procedure until getting Lyndon words in $\mathcal{L}_{i r r} \bar{Y}$.

The same treatment works for any $\ell^{\prime} \in \mathcal{L} y n X-X$.

For any $\Phi \in d m(A)$, by Proposition 16, one also has $\operatorname{ker} \phi=\operatorname{ker} \zeta=\mathcal{R}_{X}$. That means, for any irreducible Lyndon words $l \neq l^{\prime}$,

$$
\phi(l)=\phi\left(l^{\prime}\right) \quad \Longleftrightarrow \quad \zeta(l)=\zeta\left(l^{\prime}\right) .
$$

Let us state then the following

Lemma 20. Let $\Phi \in d m(A)$. Let us define the map

$$
\varphi: \mathcal{Z} \longrightarrow A
$$

as follows

$$
\forall l \in \mathcal{L}_{i r r} X, \quad \varphi(\zeta(l)):=\phi(l) .
$$

Then $\varphi$ is an algebra homomorphism and $\{\varphi(\zeta(l))\}_{l \in \mathcal{L}_{i r r} X}$ are generators of $A$.

Thus, for any $\theta \in \mathcal{Z}$ there exist the coefficients $\left\{\alpha_{l_{1}, \ldots, l_{n}}\right\}_{l_{1}, \ldots, l_{n} \in \mathcal{L}_{i r r} X}^{n \in \mathbb{N}}$ in $A$ such that (see Proposition 17 and Lemme 20)

$$
\varphi(\theta)=\sum_{n \geq 0} \sum_{l_{1}, \ldots, l_{n} \in \mathcal{L}_{i r r} X} \alpha_{l_{1}, \ldots, l_{n}} \varphi\left(\zeta\left(l_{1}\right)\right) \ldots \varphi\left(\zeta\left(l_{n}\right)\right) .
$$

In particular, since for any $w \in X^{*}, \zeta_{\varpi}(w)$ belongs to $\mathcal{Z}$ (see Corollary 3 ) then $\varphi\left(\zeta_{\omega}(w)\right)$ is well defined and $\varphi\left(\zeta_{\omega}(w)\right)$ can be expressed as polynomial on convergent polyzêtas with coefficients in $A$ :

Lemma 21. With the notations in Lemma 20, one has

$$
\forall w \in X^{*}, \quad \varphi\left(\zeta_{\varpi}(w)\right)=\sum_{\substack{u, v \in X^{*} \\ u v=w}}\left\langle e^{C} \mid v\right\rangle \zeta_{\omega}(u) .
$$

Proof. The expected result follows by identifying coefficients in $\Phi=Z_{\uplus} e^{C}$. 
Finally, we can state the following

Theorem 18. For any $\Phi \in d m(A)$, there exists an unique algebra homomorphism

$$
\varphi: \mathcal{Z} \longrightarrow A
$$

such that $\Phi$ is computed from $Z_{\varpi}$ by applying $\varphi$ to each coefficient :

$$
\Phi=\sum_{w \in X^{*}} \varphi\left(\zeta_{\varpi}(w)\right) w=\prod_{l \in \mathcal{L} y n X-X}^{\searrow} e^{\varphi(\zeta(l)) \hat{\imath}} .
$$

Remark 2. 1. In this work, neither the question deciding any real number belongs to $\mathcal{Z}$ or not nor the question expliciting $\left\{\alpha_{l_{1}, \ldots, l_{n}}\right\}_{l_{1}, \ldots, l_{n} \in \mathcal{L}_{i r r} X}^{n}$ in (86), are considered.

2. Now, by considering the commutative indeterminates $t_{1}, t_{2}, t_{3}, \ldots$, let $A$ be the $\mathbb{Q}$-algebra obtained by specializing $\mathbb{Q}\left[t_{1}, t_{2}, t_{3}, \ldots\right]$ at $t_{1}=\mathrm{i} \pi$ :

$$
A=\mathbb{Q}[\mathrm{i} \pi]\left[t_{2}, t_{3}, \ldots\right] .
$$

Neither the Lie exponential series $e^{\mathrm{i} \pi x_{0}}$ nor $e^{\mathrm{i} \pi x_{1}}$ does belong to $d m(A)$ but it belongs to $\mathrm{Gal}(D E)$. In particular, it figures in the modromies (see Section 3.2.2) or in the functional relations (see (45) and (46)) of polylogarithms and in the hexagonal relation of polyzêtas (see Proposition 6).

3. Applying Baker-Campbell-Hausdorff formula [6] to Proposition 6 we get, at orders 2 and 3 as examples, the famous Euler's formula saying $\zeta(2)$ is an algebraic number over $A=\mathbb{Q}[\mathrm{i} \pi]$ :

$$
\begin{array}{rll}
\zeta(2)+\frac{(\mathrm{i} \pi)^{2}}{6}=0 & \text { (order } 2), \\
\zeta(3)-\zeta(2,1) & =0 & \text { (order 3, imaginary part). }
\end{array}
$$

Therefore, the first comming in mind homomorphism

$$
\varphi: \mathcal{Z} \longrightarrow A
$$

maps, at least $\zeta(2)$ to $\varphi(\zeta(2))=\pi^{2} / 6$.

4. For this reason, in [30], we have to consider the $\mathbb{Q}$-algebra generated by $\mathrm{i} \pi$ and by other A-irreducible polyzêtas obtained in [32, 34, 3, 49] (and such algebra is denoted in this work by A). This algebra came up from the studies of monodromies [32, 35], as already shown in (70), and the Kummer type functional equations of polylogarithms [32, 36], as already shown in (44)-(46). In particular, by (46), we get for example [36, 32],

$$
\begin{aligned}
\operatorname{Li}_{2,1} \frac{1}{t}= & -\frac{(\mathrm{i} \pi)^{2}}{2} \log t+\mathrm{i} \pi\left(\zeta(2)-\frac{\log ^{2} t}{2}-\mathrm{Li}_{2} t\right) \\
& -\mathrm{Li}_{2,1} t+\mathrm{Li}_{3} t-\log t \mathrm{Li}_{2} t+\zeta(3)-\frac{\log ^{3} t}{6} .
\end{aligned}
$$

specializing $t=1$, the real part of this leads again to the Euler's indentity (89). 


\section{Concluding remarks : complete description of $\operatorname{ker} \zeta$ and structure of polyzêtas}

For the same raison as already said in Remark 2(2), let us consider now ${ }^{26}$ the commutative indeterminates $t_{1}, t_{2}, t_{3}, \ldots$.

Let $A$ be the $\mathbb{Q}$-algebra obtained by specializing $\mathbb{Q}\left[t_{1}, t_{2}, t_{3}, \ldots\right]$ at $t_{1}=\mathrm{i} \pi$ :

$$
A=\mathbb{Q}[\mathrm{i} \pi]\left[t_{2}, t_{3}, \ldots\right]
$$

\subsection{A conjecture by Pierre Cartier}

Definition $15([8,43])$. Let $D M(A)$ denotes the set of $\Phi \in A\langle\langle X\rangle\rangle$ such that

$$
\langle\Phi \mid \epsilon\rangle=1, \quad\left\langle\Phi \mid x_{0}\right\rangle=\left\langle\Phi \mid x_{1}\right\rangle=0, \quad \Delta_{\varpi} \Phi=\Phi \otimes \Phi
$$

and such that, for

$$
\bar{\Psi}=\exp \left(-\sum_{n \geq 2}\left\langle\pi_{Y} \Phi \mid y_{n}\right\rangle \frac{\left(-y_{1}\right)^{n}}{n}\right) \pi_{Y} \Phi \in A\langle\langle Y\rangle\rangle,
$$

then $\Delta_{\mathbf{4}} \bar{\Psi}=\bar{\Psi} \otimes \bar{\Psi}$.

Since $D M(A)$ contains already $Z_{\sqcup}$ then for $\Phi \in D M(A)$, by Theorem 13, there exists $C \in \mathcal{L}_{i e_{A}}\langle\langle X\rangle\rangle$ verifying

$$
\left\langle e^{C} \mid \epsilon\right\rangle=1 \quad \text { and } \quad\left\langle e^{C} \mid x_{0}\right\rangle=\left\langle e^{C} \mid x_{1}\right\rangle=0
$$

such that

$$
\Phi=Z_{\sqcup} e^{C}
$$

and such that

$$
\begin{aligned}
& \Psi=B^{\prime}\left(y_{1}\right) \pi_{Y} \Phi=\exp \left(-\sum_{k \geq 2} \zeta(k) \frac{\left(-y_{1}\right)^{k}}{k}\right) \pi_{Y} \Phi \\
& \bar{\Psi}=\exp \left(-\sum_{n \geq 2}\left\langle\pi_{Y} \Phi \mid y_{n}\right\rangle \frac{\left(-y_{1}\right)^{n}}{n}\right) \pi_{Y} \Phi .
\end{aligned}
$$

By construction (see Definition 11 and Theorem 13), such $\Phi$ and $\Psi$ are group-like (for the co-products $\Delta_{\sqcup}$ and $\Delta_{\sqcup+}$ respectively) and here, $\bar{\Psi}$ must be also group-like (for the co-product $\Delta_{\mathbf{m}}$ ). If such a Lie series $C$ exists then it is unique, due to the fact that $e^{C}=\Phi Z_{\sqcup}^{-1}$, and it is group-like (for the co-product $\left.\Delta_{\sqcup}\right)$.

${ }^{26}$ We do not consider in any case $A=\mathbb{Q}$ as in previous versions. 
Corollary 11 (conjectured by Cartier, [8]). For any $\Phi \in D M(A)$, there exists an unique algebra homomorphism ${ }^{27}$

$$
\bar{\varphi}: \mathcal{Z} \longrightarrow A
$$

such that $\Phi$ is computed from $Z_{\amalg}$ by applying $\bar{\varphi}$ to each coefficient.

Proof. By Theorem 18, use the fact

$$
D M(\mathbb{Q}) \subseteq D M(A) \subseteq d m(A) .
$$

\subsection{Arithmetical nature of $\gamma$}

By Theorem 14, under the assumption that the Euler constant, $\gamma$, does not belong to a commutative $\mathbb{Q}$-algebra $A$ then $\gamma$ does not verify any polynomial with coefficients in $A$ among the convergent polyzêtas. It follows then,

Corollary 12. If $\gamma \notin A$ then it is transcendental over the A-algebra generated by the convergent polyzêtas.

Or equivalently, by contraposition,

Corollary 13. If there exists a polynomial relation with coefficients in A among the Euler constant, $\gamma$, and the convergent polyzêtas then $\gamma \in A$.

Therefore,

Corollary 14. If the Euler constant, $\gamma$, does not belong to $A$ then $\gamma$ is not algebraic over $A$.

Remark 3. 1. In the same spirit of Theorem 11, let $\zeta_{ \pm+}^{T}$ be the regularization morphism ${ }^{28}$ from $(\mathbb{Q}\langle Y\rangle, \pm)$ to $(\mathbb{R},$.$) mapping y_{1}$ to $T$. Let $Z_{t+}^{T}$ be the noncommutative generating series of polyzêtas regulariszed with respect to $\zeta_{\mathrm{L}}^{T}$. Thus, as in Theorem 11 and by infinite factorization by Lyndon words, we also get

$$
Z_{t+}^{T}:=\sum_{w \in X^{*}} \zeta_{t+1}^{T}(w) w=e^{T y_{1}} Z_{ \pm+} .
$$

2. Now let us consider the regularization, for $N \rightarrow+\infty$ and with respect to $\zeta_{ \pm+ \pm}^{T}$, of the power series Const $(N)$ given in (50) as

$$
B^{T}\left(y_{1}\right)=e^{T y_{1}} B^{\prime}\left(y_{1}\right)
$$

\footnotetext{
${ }^{27}$ See Remark 2(3) to have an example of $\bar{\varphi}$.

${ }^{28}$ This is a symbolic regularization and does not yet have an analytical justification as it is done, separately, for $\zeta_{\omega}$ and $\zeta_{\boldsymbol{L}}$ in Section 3.1.2 as finite parts of the asymptotic expansions, in different scales of comparison, of $\operatorname{Li}_{x_{1}}(z)$, for $z \rightsquigarrow 1$, and $\mathrm{H}_{y_{1}}(N)$, for $N \rightarrow \infty$, respectively.
} 
As in Corollary 6, we always get

$$
Z_{\uplus}^{T}=B^{T}\left(y_{1}\right) \pi_{Y} Z_{\varpi} \quad \Longleftrightarrow \quad Z_{\uplus}=B^{\prime}\left(y_{1}\right) \pi_{Y} Z_{山} .
$$

Hence, roughly speaking, for the quasi-shuffle product, the symbolic regularization to $T$ is also "equivalent" to the regularization to 0 .

3. Again, as in Corollary 12, if $T \notin A$ then $T \notin \bar{A}$.

A contrario, as in Corollary 13, if there exists a polynomial relation with coefficients in $A$ among $T$ and convergent polyzêtas then $T \in A$.

\subsection{Structure and arithmetical nature of polyzêtas}

Once again, let us consider

$$
\begin{aligned}
& \left(A_{1}, \omega\right)=\left(A \epsilon \oplus x_{0} A\langle X\rangle x_{1}, \omega\right) \\
& \cong A[\mathcal{L} y n X-X], \omega) \\
& =\left(\mathcal{R}_{X}, ш\right) \oplus\left(A\left[\mathcal{L}_{i r r} X\right], ш\right), \\
& \left(A_{2}, \sqcup\right)=\left(A \epsilon \oplus\left(\bar{Y}-\left\{y_{1}\right\}\right) A\langle\bar{Y}\rangle, \omega\right) \\
& \cong\left(A\left[\mathcal{L} y n \bar{Y}-y_{1}\right], \uplus\right) \\
& =\left(\mathcal{R}_{\bar{Y}}, \omega\right) \oplus\left(A\left[\mathcal{L}_{i r r} \bar{Y}\right], \omega\right)
\end{aligned}
$$

(see Definition 10, Lemma 16, Definition 13). Then $\left(A_{1}, ш\right) \cong\left(A_{2}\right.$, ш $)[32,33]$.

Let us consider again the following algebra morphism (see Proposition 9)

$$
\begin{aligned}
\zeta: \begin{array}{c}
\left(A_{2}, \boldsymbol{\omega}\right) \\
\left(A_{1}, \omega\right)
\end{array} & \longrightarrow(\mathbb{R}, .) \\
y_{r_{1}} \ldots y_{r_{k}} & \longmapsto \sum_{n_{1}>\ldots>n_{k}>0} \frac{1}{n_{1}^{r_{1}} \ldots n_{k}^{r_{k}}} .
\end{aligned}
$$

Lemma 22. The image of the algebra morphism $\zeta$ is $\mathcal{Z}$.

Let us make precise the structure of $\mathcal{Z}$ and the arithmetical nature of polyzêtas :

As consequences of the propositions 15,16 and 17 , by taking $\Phi=Z_{\amalg}$, we have

$$
\begin{array}{rlll}
\operatorname{Im} \zeta=\zeta\left(\mathrm{A}\left[\mathcal{L}_{i r r} \bar{Y}\right]\right) & \text { and } & \operatorname{ker} \zeta=\mathcal{R}_{\bar{Y}} \\
\text { (resp. } & \operatorname{Im} \zeta=\zeta\left(\mathrm{A}\left[\mathcal{L}_{i r r} X\right]\right) & \text { and } & \left.\operatorname{ker} \zeta=\mathcal{R}_{X}\right) .
\end{array}
$$

By Corollary $10, \operatorname{ker} \zeta$ is an ideal generated by the homogenous polynomials of degree $\geq 2$. Hence, the quotien $A_{1} / \mathcal{R}_{X}$ or $A_{2} / \mathcal{R}_{\bar{Y}}$ (the source by the kernel of $\zeta$ ) is graded [6] and it is isomorphic to $\operatorname{Im} \zeta$.

Therefore, by Lemma 22 and Proposition 17, we obtain respectively the following direct consequences

Theorem 19 (Structure of polyzêtas). The A-algebra $\mathcal{Z}$ is 
1. isomorphic to the graded algebra $\left(A_{1} / \mathcal{R}_{X}, \omega\right)$, or equivalently, $\left(A_{2} / \mathcal{R}_{\bar{Y}}, \uplus\right)$.

2. freely generated by the $A$-irreducible polyzêtas $\{\zeta(l)\}_{l \in \mathcal{L}_{i r r} \bar{Y}}\left(\right.$ resp. $\left.\{\zeta(l)\}_{l \in \mathcal{L}_{i r r} X}\right)$.

For any $p \geq 2$, let

$$
\mathcal{Z}_{p}=\operatorname{span}_{\mathbb{Q}}\left\{\zeta(w)\left|w \in x_{0} X^{*} x_{1},\right| w \mid=p\right\} .
$$

By definition of graded algebra [6], Theorem 19 means also that

$$
\mathcal{Z}=A \oplus \bigoplus_{p \geq 2} \mathcal{Z}_{p}
$$

and there is no linear relation among elements of different $\mathcal{Z}_{p}$ ([8] conjecture $\mathrm{C} 1,[48])$.

Thus, if $\theta$ is a ( $A$-irreducible) polyzêta verifying the following algebraic equation

$$
\theta^{n}+a_{n-1} \theta^{n-1}+\ldots+a_{0}=0
$$

then $\theta=0$ because $\mathcal{Z}_{p_{1}} \mathcal{Z}_{p_{2}} \subset \mathcal{Z}_{p_{1}+p_{2}}$, for $p_{1}, p_{2} \geq 2$, and each monomial in (110) is then of different weight. By consequence,

Corollary 15. Any (A-irreducible) polyzêta $\theta$ is a transcendental over $\mathbb{Q}$.

Remark 4. In this work, neither the study of $\operatorname{dim} \mathcal{Z}_{p}$ [50] (see also [8], conjecture C2) nor the estimate of the number of A-irreducible polyzêtas generating $\mathcal{Z}_{p}$, are discussed knowing the $A$-irreducible polyzêtas form transcendence basis of the A-algebra $\mathcal{Z}$.

\section{Annexe A : pair of bases in duality and proof of Theorem 2}

\subsection{Preliminary results}

Let $\mathbb{Q}\langle Y\rangle$ be equipped the concatenation and the quasi-shuffle, $\leftarrow$, defined by

$$
\begin{array}{rlrl}
\forall y_{i}, y_{j} \in Y=\left\{y_{i}\right\}_{i \geq 1}, \forall u, v \in Y^{*}, & & y_{i} u \uplus y_{j} v=y_{i}\left(u \uplus y_{j} v\right)+y_{i+j}\left(y_{i} u \uplus v\right), \\
\forall w \in Y^{*}, & w \uplus 1_{Y^{*}}=1_{Y^{*}} \uplus w=w,
\end{array}
$$

or by its associated co-product, $\Delta_{\uplus+}$, defined by

$$
\forall y_{k} \in Y, \quad \Delta_{\boldsymbol{\Psi}}\left(y_{k}\right)=y_{k} \otimes 1+1 \otimes y_{k}+\sum_{i+j=k} y_{i} \otimes y_{j} .
$$

satistying, for any $u, v, w \in Y^{*},\left\langle u \otimes v \mid \Delta_{\uplus}(w)\right\rangle=\langle u \uplus v \mid w\rangle$.

Lemma 23. Let $S_{1}, \ldots, S_{n}$ be proper formal power series in $\mathbb{Q}\langle\langle Y\rangle\rangle$. Let $P_{1}, \ldots, P_{m}$ be primitive elements ${ }^{29}$ in $\mathbb{Q}\langle Y\rangle$, for the co-product $\amalg$.

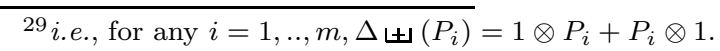


1. If $n>m$ then $\left\langle S_{1} \uplus \ldots \uplus S_{n} \mid P_{1} \ldots P_{m}\right\rangle=0$.

2. If $n=m$ then

$$
\left\langle S_{1} \uplus \ldots \uplus S_{n} \mid P_{1} \ldots P_{n}\right\rangle=\sum_{\sigma \in \mathfrak{S}_{n}} \prod_{i=1}^{n}\left\langle S_{i} \mid P_{\sigma(i)}\right\rangle .
$$

3. If $n<m$ then, by considering the language $\mathcal{M}$ over $\mathcal{A}=\left\{P_{1}, \ldots, P_{m}\right\}$

$$
\mathcal{M}=\left\{w \in \mathcal{A}^{*}\left|w=P_{j_{1}} \ldots P_{j_{|w|},} j_{1}<\ldots<j_{|w|},\right| w \mid \geq 1\right\}
$$

and the morphism $\mu: \mathbb{Q}\langle\mathcal{A}\rangle \longrightarrow \mathbb{Q}\langle Y\rangle$, one has :

$$
\left\langle S_{1} \uplus \ldots \uplus S_{n} \mid P_{1} \ldots P_{m}\right\rangle=\sum_{\substack{w_{1}, \ldots, w_{m} \in \mathcal{M} \\\left|w_{1}+\ldots+\ldots w_{n}\right|=m \\ \forall i, j=1, \ldots m, \operatorname{alp}\left(w_{i}\right) \cap \operatorname{lalp}\left(w_{j}\right)=\emptyset}} \prod_{i=1}^{n}\left\langle S_{i} \mid \mu\left(w_{i}\right)\right\rangle .
$$

Proof. On one hand, since the $P_{i}$ 's are primitive then

$$
\Delta_{ \pm+}^{(n-1)}\left(P_{i}\right)=\sum_{p+q=n-1} 1^{\otimes p} \otimes P_{i} \otimes 1^{\otimes q} .
$$

On the other hand, $\left\langle S_{1} \uplus \ldots \uplus S_{n} \mid P_{1} \ldots P_{m}\right\rangle=\left\langle S_{1} \otimes \ldots \otimes S_{n} \mid \Delta_{\uplus+}^{(n-1)}\left(P_{1} \ldots P_{m}\right)\right\rangle$ and $\Delta_{ \pm+}^{(n-1)}\left(P_{1} \ldots P_{m}\right)=\Delta_{\sqcup+}^{(n-1)}\left(P_{1}\right) \ldots \Delta_{\sqcup+}^{(n-1)}\left(P_{m}\right)$. Hence,

$$
\left\langle S_{1} \uplus \ldots \uplus S_{n} \mid P_{1} \ldots P_{m}\right\rangle=\left\langle\bigotimes_{i=1}^{n} S_{i} \mid \prod_{i=1}^{m} \sum_{p+q=n-1} 1^{\otimes p} \otimes P_{i} \otimes 1^{\otimes q}\right\rangle .
$$

1. For $n>m$, by expanding $\Delta_{t+}^{(n-1)}\left(P_{1}\right) \ldots \Delta_{t+}^{(n-1)}\left(P_{m}\right)$, one obtains a sum of tensors contening at least one factor equal to 1 . For $j=1, . ., n$, the formal power series $S_{j}$ is proper and the result follows immediatly.

2. For $n=m$, since

$$
\prod_{i=1}^{n} \Delta_{\sqcup+}^{(n-1)}\left(P_{i}\right)=\sum_{\sigma \in \mathfrak{S}_{n}} \bigotimes_{i=1}^{n} P_{\sigma(i)}+Q
$$

where $Q$ is sum of tensors contening at least one factor equal to 1 and the $S_{j}$,'s are proper then $\left\langle S_{1} \otimes \ldots \otimes S_{n} \mid Q\right\rangle=0$. Thus, the result follows.

3. For $n<m$, en tenant compte que, for $j=1, . ., n$, formal power series $S_{j}$ is proper, the expected follows by expanding the product

$$
\prod_{i=1}^{m} \Delta_{+ \pm}^{(n-1)}\left(P_{i}\right)=\prod_{i=1}^{m} \sum_{p+q=n-1} 1^{\otimes p} \otimes P_{i} \otimes 1^{\otimes q} .
$$


Proposition 18. 1. We have

$$
\log \left(\sum_{w \in Y^{*}} w \otimes w\right)=\sum_{w \in Y^{+}} w \otimes \pi_{1}(w)=\sum_{w \in Y^{+}} \pi_{1}^{*}(w) \otimes w,
$$

where $\pi_{1}^{*}$ is the adjoint of $\pi_{1}$ and they are given by

$$
\begin{aligned}
& \left.\pi_{1}(w)=\sum_{k \geq 1} \frac{(-1)^{k-1}}{k} \sum_{u_{1}, \ldots, u_{k} \in Y^{+}}\langle w| u_{1} \leftarrow \ldots \text { เ } u_{k}\right\rangle u_{1} \ldots u_{k}, \\
& \pi_{1}^{*}(w)=\sum_{k \geq 1} \frac{(-1)^{k-1}}{k} \sum_{u_{1}, \ldots, u_{k} \in Y^{+}}\left\langle w \mid u_{1} \ldots u_{k}\right\rangle u_{1} \leftarrow \ldots \text { เ } u_{k} .
\end{aligned}
$$

In particular, for any $y_{k} \in Y$, one has

$$
\pi_{1}\left(y_{k}\right)=y_{k}+\sum_{l \geq 2} \frac{(-1)^{l-1}}{l} \sum_{\substack{j_{1}, \ldots, j_{l} \geq 1 \\ j_{1}+\ldots+j_{l}=k}} y_{j_{1}} \ldots y_{j_{l}} \quad \text { and } \quad \pi_{1}^{*}\left(y_{k}\right)=y_{k}
$$

2. For any $w \in Y^{*}$, we have

$$
\begin{aligned}
w & =\sum_{k \geq 0} \frac{1}{k !} \sum_{u_{1}, \ldots, u_{k} \in Y^{*}}\left\langle w \mid u_{1} \uplus \ldots \uplus u_{k}\right\rangle \pi_{1}\left(u_{1}\right) \ldots \pi_{1}\left(u_{k}\right), \\
& =\sum_{k \geq 0} \frac{1}{k !} \sum_{u_{1}, \ldots, u_{k} \in Y^{*}}\left\langle w \mid u_{1} \ldots u_{k}\right\rangle \pi_{1}^{*}\left(u_{1}\right) \leftarrow \ldots \text { เ } \pi_{1}^{*}\left(u_{k}\right) .
\end{aligned}
$$

Proof. 1. Expanding the logarithm, we have

$$
\begin{aligned}
& \log \left(\sum_{w \in Y^{*}} w \otimes w\right)=\sum_{k \geq 1} \frac{(-1)^{k-1}}{k}\left(\sum_{w \in Y^{+}} w \otimes w\right)^{k} \\
& =\sum_{k \geq 1} \frac{(-1)^{k-1}}{k} \sum_{u_{1}, \ldots, u_{k} \in Y^{+}}\left(u_{1} \uplus \ldots \uplus u_{k}\right) \otimes u_{1} \ldots u_{k} \\
& =\sum_{w \in Y^{+}} w \otimes\left(\sum_{k \geq 1} \frac{(-1)^{k-1}}{k}\right. \\
& \left.\sum_{u_{1}, \ldots, u_{k} \in Y^{+}}\left\langle w \mid u_{1} \uplus \ldots \uplus u_{k}\right\rangle u_{1} \ldots u_{k}\right) \text {. }
\end{aligned}
$$

In the same way,

$$
\begin{aligned}
\log \left(\sum_{w \in Y^{*}} w \otimes w\right)= & \sum_{w \in Y^{+}}\left(\sum_{k \geq 1} \frac{(-1)^{k-1}}{k}\right. \\
& \left.\sum_{u_{1}, \ldots, u_{k} \in Y^{+}}\left\langle w \mid u_{1} \ldots u_{k}\right\rangle u_{1} \text { เ } \ldots \text { เ } u_{k}\right) \otimes w .
\end{aligned}
$$

Thus, the expressions of $\pi_{1}(w)$ and $\pi_{1}^{*}(w)$ follow immediatly. 
2. Since exp and log are mutually inverse then, by the previous results, one has

$$
\begin{aligned}
\sum_{w \in Y^{*}} w \otimes w= & \sum_{k \geq 0} \frac{1}{k !}\left(\sum_{w \in Y^{+}} w \otimes \pi_{1}(w)\right)^{k} \\
= & \sum_{k \geq 0} \frac{1}{k !} \sum_{u_{1}, \ldots, u_{k} \in Y^{+}}\left(u_{1} \uplus \ldots \uplus u_{k}\right) \otimes\left(\pi_{1}\left(u_{1}\right) \ldots \pi_{1}\left(u_{k}\right)\right) \\
= & \sum_{w \in Y^{+}} w \otimes\left(\sum_{k \geq 1} \frac{1}{k !}\right. \\
& \left.\sum_{u_{1}, \ldots, u_{k} \in Y^{+}}\left\langle w \mid u_{1} \uplus \ldots \uplus u_{k}\right\rangle \pi_{1}\left(u_{1}\right) \ldots \pi_{1}\left(u_{k}\right)\right) .
\end{aligned}
$$

In the same way,

$$
\begin{aligned}
\sum_{w \in Y^{*}} w \otimes w= & \sum_{k \geq 0} \frac{1}{k !} \sum_{u_{1}, \ldots, u_{k} \in Y^{+}}\left(\pi_{1}^{*}\left(u_{1}\right) \uplus \ldots \uplus \pi_{1}^{*}\left(u_{k}\right)\right) \otimes\left(u_{1} \ldots u_{k}\right) \\
= & \sum_{w \in Y^{+}}\left(\sum_{k \geq 0} \frac{1}{k !}\right. \\
& \left.\sum_{u_{1}, \ldots, u_{k} \in Y^{+}}\left\langle w \mid u_{1} \ldots u_{k}\right\rangle \pi_{1}^{*}\left(u_{1}\right)+\ldots \uplus \pi_{1}^{*}\left(u_{k}\right)\right) \otimes w .
\end{aligned}
$$

It follows then the expected result.

\subsection{Pair of bases in duality}

Definition 16. Let $\left\{\Sigma_{l}\right\}_{l \in \mathcal{L} y n Y}$ be the family of $\mathcal{L}_{e_{\mathbb{Q}}}\langle Y\rangle$ obtained as follows

$$
\begin{aligned}
\Sigma_{y_{k}} & =\pi_{1}\left(y_{k}\right) \quad \text { for } \quad k \geq 1, \\
\Sigma_{l} & =\left[\Sigma_{s}, \Sigma_{r}\right] \text { for } \quad l \in \mathcal{L} y n X, \text { standard factorization of } l=(s, r),
\end{aligned}
$$

and the family $\left\{\Sigma_{w}\right\}_{w \in Y^{*}}$ of $\mathcal{U}\left(\mathcal{L}_{e_{\mathbb{Q}}}\langle Y\rangle\right)$ (viewed as a $\mathbb{Q}$-module) obtained as follows

$$
\begin{aligned}
& \Sigma_{l}=1 \text { for } l=1_{Y^{*}}, \\
& \Sigma_{w}=\Sigma_{l_{1}}^{i_{1}} \ldots \Sigma_{l_{k}}^{i_{k}} \text { for } w=l_{1}^{i_{1}} \ldots l_{k}^{i_{k}}, l_{1}>\ldots>l_{k}, l_{1} \ldots, l_{k} \in \mathcal{L} y n Y .
\end{aligned}
$$

Let $\left\{\check{\Sigma}_{w}\right\}_{w \in Y^{*}}$ be the family of the quasi-shuffle algebra (viewed as a $\mathbb{Q}$-module) obtained by duality with $\left\{\Sigma_{w}\right\}_{w \in Y^{*}}$ :

$$
\forall u, v \in Y^{*}, \quad\left\langle\check{\Sigma}_{v} \mid \Sigma_{u}\right\rangle=\delta_{u, v} .
$$

Proposition 19. 1. For $l \in \mathcal{L} y n Y$, the polynomial $\Sigma_{l}$ is upper triangular :

$$
\Sigma_{l}=l+\sum_{v>w,(v)=(l)} c_{v} v .
$$


2. The families $\left\{\Sigma_{w}\right\}_{w \in Y^{*}}$ and $\left\{\check{\Sigma}_{w}\right\}_{w \in Y^{*}}$ are upper and lower triangular respectively. On other words, for any $w \in Y^{+}$, one has

$$
\Sigma_{w}=w+\sum_{v>w,(v)=(w)} c_{v} v \text { and } \check{\Sigma}_{w}=w+\sum_{v<w,(v)=(w)} d_{v} v,
$$

where, for any $y_{k} \in Y$ and $w \in Y^{*},(w)$ denotes the degree of $w$ and $\left(y_{k}\right)=$ $\operatorname{deg}\left(y_{k}\right)=k$.

Proof. 1. Let us prove it by induction on the length of $l$ :

- The result is immediat for $l \in Y$.

- The result is suppose verified for any $l \in \mathcal{L} y n Y \cap Y^{k}$ and $0 \leq k \leq N$.

- At $N+1$, by the standard factorization $\left(l_{1}, l_{2}\right)$ of $l$, one has, by definition, $\Sigma_{l}=\left[\Sigma_{l_{1}}, \Sigma_{l_{2}}\right]$ and $l_{2} l_{1}>l_{1} l_{2}=l$. By induction hypothesis,

$$
\begin{array}{r}
\Sigma_{l_{1}}=l_{1}+\sum_{\substack{\left.v>l_{1} \\
v\right)=\left(l_{1}\right)}} c_{v} v \quad \text { and } \Sigma_{l_{2}}=l_{2}+\sum_{\substack{u>l_{2} \\
(v)=\left(l_{2}\right)}} d_{u} u, \\
\Rightarrow \quad \Sigma_{l}=l+\sum_{\substack{w>l \\
(w)=(l)}} e_{w} w,
\end{array}
$$

getting $e_{w}$ 's from $c_{v}$ 's and $d_{u}$ 's. Actually, the Lie bracket gives

$$
\begin{aligned}
& \Sigma_{l}=\left[l_{1}, l_{2}\right] \\
& +\sum_{\substack{\left.u>l_{2} \\
v\right)=\left(l_{2}\right)}} d_{u} l_{1} u+\sum_{\substack{\left.v>l_{1}, u>l_{2} \\
v\right)=\left(l_{1}\right),(u)=\left(l_{2}\right)}} c_{v} d_{u} v u \\
& \text { - } \sum_{\substack{\left.v>l_{1} \\
v\right)=\left(l_{1}\right)}} c_{v} l_{2} v-\sum_{\substack{v>l_{1}, u>l_{2} \\
(v)=\left(l_{2}\right),(u)=\left(l_{1}\right)}} c_{v} d_{u} u v \\
& +\sum_{\substack{\left.u>l_{1} l_{2} \\
v\right)=\left(l_{1} l_{2}\right)}} d_{u}^{\prime} u+\sum_{\substack{v u>l_{1} l_{2} \\
(v u)=\left(l_{1} l_{2}\right)}} c_{v} d_{u} v u \\
& -\sum_{\substack{v>l_{2} l_{1} \\
(v)=\left(l_{2} l_{1}\right)}} c_{v}^{\prime} v-\sum_{\substack{u v>l_{2} l_{1} \\
(u v)=\left(l_{2} l_{1}\right)}} c_{v} d_{u} u v \\
& =\left[l_{1}, l_{2}\right] \\
& +\sum_{\substack{u>l \\
(v)=(l)}} d_{u}^{\prime} u+\sum_{\substack{v u>l \\
(v u)=(l)}} c_{v} d_{u} v u \\
& -\sum_{\substack{v>l_{2} l_{1}>l \\
(v)=(l)}} c_{v}^{\prime} v-\sum_{\substack{u v>l_{2} l_{1}>l \\
(u v)=(l)}} c_{v} d_{u} u v .
\end{aligned}
$$

Hence, the conclusion follows.

2. Let $w=l_{1} \ldots l_{k}$, with $l_{1}>\ldots>l_{k}$ and $l_{1}, \ldots, l_{k} \in \mathcal{L} y n Y$. By (ii),

$$
\Sigma_{l_{i}}=l_{i}+\sum_{\substack{v>l_{i} \\(v)=\left(l_{i}\right)}} c_{i, v} v \text { and } \Sigma_{w}=l_{1} \ldots l_{k}+\sum_{\substack{u>w \\(v)=(w)}} d_{u} u
$$


where the $d_{u}$ 's are obtained from the $c_{i, v}$ 's. Hence, the family $\left\{\Sigma_{w}\right\}_{w \in Y^{*}}$ is upper triangular and, by duality, the family $\left\{\check{\Sigma}_{w}\right\}_{w \in Y^{*}}$ is lower triangular.

Theorem 20. 1. The family $\left\{\Sigma_{l}\right\}_{l \in \mathcal{L} y n Y}$ forms a basis of the free Lie algebra.

2. The family $\left\{\Sigma_{w}\right\}_{w \in Y^{*}}$ forms a basis of the free associative algebra $\mathbb{Q}\langle Y\rangle$.

3. The family $\left\{\check{\Sigma}_{w}\right\}_{w \in Y^{*}}$ generate freely the quasi-shuffle algebra.

4. The family $\left\{\check{\Sigma}_{l}\right\}_{l \in \mathcal{L} y n Y}$ forms a transcendence basis of the quasi-shuffle algebra.

Proof. The family $\left\{\Sigma_{l}\right\}_{l \in \mathcal{L} y n Y}$ of upper triangular polynomials is free and then, by a theorem of Viennot, we get the first result. The second is a direct consequence of the Poincaré-Birkhoff-Witt theorem. By the Cartier-Quillen-MilnorMoore theorem, we get the third one and the last one is also obtained as consequence of the constructions of the families $\left\{\check{\Sigma}_{l}\right\}_{l \in \mathcal{L} y n Y}$ and $\left\{\check{\Sigma}_{w}\right\}_{w \in Y^{*}}$ of lower triangular polynomials.

Now, let us clarify the basis $\left\{\check{\Sigma}_{w}\right\}_{w \in Y^{*}}$ and then the transcendence basis $\left\{\check{\Sigma}_{l}\right\}_{l \in \mathcal{L} y n Y}$ of the quasi-shuffle algebra $(\mathbb{Q}\langle Y\rangle, \omega)$ as follows

Theorem 21. We have

1. For $w=1_{Y^{*}}, \check{\Sigma}_{w}=1$.

2. For any $w=l_{1}^{i_{1}} \ldots l_{k}^{i_{k}}$, with $l_{1}, \ldots, l_{k} \in \mathcal{L} y n Y$ and $l_{1}>\ldots>l_{k}$,

$$
\check{\Sigma}_{w}=\frac{\check{\Sigma}_{l_{1}}^{\uplus i_{1}} \amalg \ldots \amalg \check{\Sigma}_{l_{k}}^{\uplus i_{k}}}{i_{1} ! \ldots i_{k} !} .
$$

3. For any $y \in Y, \check{\Sigma}_{y}=\pi_{1}^{*}(y)$.

4. For any $l=y u \in \mathcal{L} y n Y-Y, \check{\Sigma}_{l}=y \check{\Sigma}_{u}$.

Proof. $\quad 1$. Since $\Sigma_{1_{Y^{*}}}=1$ then $\check{\Sigma}_{1_{Y^{*}}}=1$.

2. Let $u=u_{1} \ldots u_{n}=l_{1}^{i_{1}} \ldots l_{k}^{i_{k}}, v=v_{1} \ldots v_{m}=h_{1}^{j_{1}} \ldots h_{p}^{j_{p}}$ with $l_{1} \ldots, l_{k}$, $h_{1}, \ldots, h_{p}, u_{1}, \ldots, u_{n}, v_{1}, \ldots, v_{m} \in \mathcal{L} y n Y, l_{1}>\ldots>l_{k}, h_{1}>\ldots>h_{p}$, $u_{1} \geq \ldots \geq u_{n}, v_{1} \geq \ldots \geq v_{m}$ and $i_{1}+\ldots+i_{k}=n, j_{1}+\ldots+j_{p}=m$. Hence, if $m \geq 2$ (resp. $n \geq 2$ ) then $v \notin \mathcal{L} y n Y$ (resp. $u \notin \mathcal{L} y n Y$ ).

Since

$$
\left\langle\check{\Sigma}_{u_{1}} \uplus \ldots \uplus \check{\Sigma}_{u_{n}} \mid \prod_{i=1}^{n} \Sigma_{u_{i}}\right\rangle=\left\langle\check{\Sigma}_{u_{1}} \otimes \ldots \otimes \check{\Sigma}_{u_{n}} \mid \Delta_{+ \pm}^{(n-1)}\left(\Sigma_{v_{1}} \ldots \Sigma_{v_{m}}\right)\right\rangle
$$

then many cases occur : 
(a) Case $n>m$. By Lemma 23(1), $\left\langle\check{\Sigma}_{u_{1}} \uplus \ldots \leftarrow \check{\Sigma}_{u_{n}} \mid \Sigma_{v_{1}} \ldots \Sigma_{v_{m}}\right\rangle=0$.

(b) Case $n=m$. By Lemma 23(2), one has

$$
\begin{aligned}
\left\langle\check{\Sigma}_{u_{1}}+\ldots+\check{\Sigma}_{u_{n}} \mid \prod_{i=1}^{n} \Sigma_{v_{i}}\right\rangle & =\sum_{\sigma \in \check{\Sigma}_{n}} \prod_{i=1}^{n}\left\langle\check{\Sigma}_{u_{i}} \mid \Sigma_{v_{\sigma(i)}}\right\rangle \\
& =\sum_{\sigma \in \check{\Sigma}_{n}} \prod_{i=1}^{n} \delta_{\check{\Sigma}_{u_{i}}, \Sigma_{v_{\sigma(i)}}} .
\end{aligned}
$$

Thus, if $u \neq v$ then $\left(u_{1}, \ldots, u_{n}\right) \neq\left(v_{1}, \ldots, v_{n}\right)$ then the second member is vanishing else, i.e. $u=v$, the second member equals 1 because the factorization by Lyndon words is unique.

(c) Case $n<m$. By Lemma 23(3), let us consider the following language over the alphabet $\mathcal{A}=\left\{\Sigma_{v_{1}}, \ldots, \Sigma_{v_{m}}\right\}$ :

$$
\mathcal{M}=\left\{w \in \mathcal{A}^{*}\left|w=\Sigma_{v_{j_{1}}} \ldots \Sigma_{v_{j_{|w|} \mid}}, j_{1}<\ldots<j_{|w|},\right| w \mid \geq 1\right\}
$$

and the morphism $\mu: \mathbb{Q}\langle\mathcal{A}\rangle \longrightarrow \mathbb{Q}\langle Y\rangle$. We get :

$$
\begin{aligned}
\left\langle\mathbf{w}_{i=1}^{n} \check{\Sigma}_{u_{i}} \mid \prod_{i=1}^{n} \Sigma_{u_{i}}\right\rangle & =\sum_{\substack{w_{1}, \ldots, w_{m} \in \mathcal{M} \\
\left|w_{1}\right|+\ldots+w_{n} \mid=m \\
\forall i, j=1, \ldots m, \operatorname{alp}\left(w_{i}\right) \cap \operatorname{lal}\left(w_{j}\right)=\emptyset}} \prod_{i=1}^{n}\left\langle\check{\Sigma}_{u_{i}} \mid \mu\left(w_{i}\right)\right\rangle \\
& =0 .
\end{aligned}
$$

Because in this product, on one hand, there exists at least one $w_{i} \in$

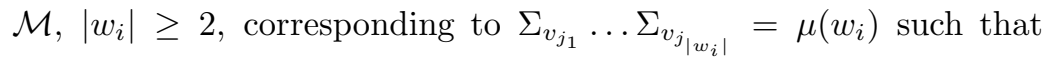
$v_{j_{1}} \geq \ldots \geq v_{j_{\left|w_{i}\right|}}$ and on other hand, $\nu_{i}:=v_{j_{1}} \ldots v_{j_{\left|w_{i}\right|}} \notin \mathcal{L} y n Y$ and $u_{i} \in \mathcal{L} y n Y$.

By consequent,

$$
\left\langle\check{\Sigma}_{u} \mid \Sigma_{v}\right\rangle=\left\langle\frac{\check{\Sigma}_{l_{1}}^{\mathbf{L} i_{1}} \pm \ldots \pm \check{\Sigma}_{l_{k}}^{\mathbf{L} i_{k}}}{i_{1} ! \ldots i_{k} !} \mid \Sigma_{h_{1}}^{j_{1}} \ldots \Sigma_{h_{p}}^{j_{p}}\right\rangle=\delta_{u, v}
$$

3. For any $l \in Y, \Sigma_{l}=\pi_{1}(l), \check{\Sigma}_{l}=\pi_{1}^{*}(l)$ and $\pi_{1}, \pi_{1}^{*}$ are mutually adjoint.

4. By decomposing bu in the PBW-Lyndon basis, we get on one hand,

$$
\begin{aligned}
b u & =\sum_{w \in Y^{*}}\left\langle\check{\Sigma}_{w} \mid b u\right\rangle \Sigma_{w}+\sum_{a w \in \mathcal{L} y n Y}\left\langle\check{\Sigma}_{a w} \mid b u\right\rangle \Sigma_{a w} \\
& + \text { sum of decreasinge products of length } \geq 2, \text { of Lie polynomials. }
\end{aligned}
$$

Since for any $a w=l_{1}^{i_{1}} \ldots l_{k}^{i_{k}} \notin \mathcal{L} y n Y(k \geq 2)$ with $l_{1}, \ldots, l_{k} \in \mathcal{L} y n Y$ and $l_{1}>\ldots>l_{k}$, then $\Sigma_{a w}=\Sigma_{l_{1}} \ldots \Sigma_{l_{k}}$. Since (see Proposition 19)

$$
\Sigma_{w}=w+\sum_{v>w,(v)=(w)} c_{v} v \text { and } \Sigma_{b w}=b w+\sum_{v>b w,(v)=(b w)} d_{v} v
$$


then, by decomposing $u$ in the PBW-Lyndon basis and then by multiplying by $b$, we get on other hand,

$$
\begin{aligned}
b u & =\sum_{w \in Y^{*}}\left\langle\check{\Sigma}_{w} \mid u\right\rangle b \Sigma_{w} \\
& =\sum_{w \in Y^{*}}\left\langle\check{\Sigma}_{w} \mid u\right\rangle\left(\Sigma_{b w}-\sum_{v>b w,(v)=(b w)} d_{v} v\right) \\
& =\sum_{w \in Y^{*}}\left\langle\check{\Sigma}_{w} \mid u\right\rangle \Sigma_{b w}-\sum_{w \in Y^{*}} d_{v>b w,(v)=(b w)} \sum_{w^{\prime} \in Y^{*}}\left\langle\check{\Sigma}_{w^{\prime}} \mid v\right\rangle \Sigma_{w^{\prime}} \\
& =\sum_{w \in Y^{*}}\left\langle\check{\Sigma}_{w} \mid u\right\rangle \Sigma_{b w}-\sum_{w \in Y^{*}} \sum_{w^{\prime} \in Y^{*}} \sum_{v>b w,(v)=(b w)} d_{v}\left\langle\check{\Sigma}_{w^{\prime}} \mid v\right\rangle \Sigma_{w^{\prime}} \\
& =\sum_{\sum_{w \in \mathcal{L} y n Y}}\left\langle\check{\Sigma}_{w} \mid u\right\rangle \Sigma_{b w}-\sum_{w^{\prime} \in \mathcal{L} y n Y}\left\langle\check{\Sigma}_{w^{\prime}} \mid v\right\rangle \Sigma_{w^{\prime}} \\
& +\operatorname{sum~of~decreasinge~products,~of~length~} \geq 2, \text { of Lie polynomials. }
\end{aligned}
$$

After splitting these two sums on two disjoint supports, one has

- for any $b w=l_{1}^{i_{1}} \ldots l_{n}^{i_{n}} \notin \mathcal{L} y n Y(n \geq 2)$ with $l_{1}, \ldots, l_{n} \in \mathcal{L} y n Y$ verifying $l_{1}>\ldots>l_{n}$ and $\Sigma_{a w}=\Sigma_{l_{1}}^{i_{n}} \ldots \Sigma_{l_{n}}^{i_{n}}$.

- for any $w^{\prime}=\lambda_{1}^{j_{1}} \ldots \lambda_{m}^{j_{m}} \notin \mathcal{L} y n Y(m \geq 2)$ with $\lambda_{1}, \ldots, \lambda_{m} \in \mathcal{L} y n Y$ verifying $\lambda_{1}>\ldots>\lambda_{m}$ and $\Sigma_{w^{\prime}}=\Sigma_{\lambda_{1}}^{j_{1}} \ldots \Sigma_{\lambda_{m}}^{j_{m}}$.

In the second sum, since each word $v$ is great than the Lyndon word $b w$ then the Lie polynomial $\Sigma_{b w}$ does not appear in the decomposition, in the PBW-Lyndon basis, of $v$. More precisely, (see Proposition 19)

$$
\begin{aligned}
\check{\Sigma}_{w^{\prime}} & =w^{\prime}+\sum_{w^{\prime}>v^{\prime},\left(w^{\prime}\right)=\left(v^{\prime}\right)} e_{v^{\prime}} v^{\prime} \quad \text { with } e_{v^{\prime}} \geq 0, \\
\Rightarrow\left\langle\check{\Sigma}_{w^{\prime}} \mid v\right\rangle & =\left\langle w^{\prime} \mid v\right\rangle+\sum_{w^{\prime}>v^{\prime},\left(w^{\prime}\right)=\left(v^{\prime}\right)} e_{v^{\prime}}\left\langle v^{\prime} \mid v\right\rangle .
\end{aligned}
$$

In particular (for $w^{\prime}=b w \in \mathcal{L} y n Y$ ), the coefficient of the Lie polynomial $\Sigma_{b w}$ in the decomposition of $v(>b w)$ is vanishing :

$$
\left\langle\check{\Sigma}_{b w} \mid v\right\rangle=\langle b w \mid v\rangle+\sum_{v>b w>v^{\prime},(b w)=\left(v^{\prime}\right)} e_{v^{\prime}}\left\langle v^{\prime} \mid v\right\rangle=0 .
$$

Thus, by identifying the coefficients in these two expressions of Lyndon word $b u$, one has $\left\langle\check{\Sigma}_{a w} \mid b u\right\rangle=\delta_{a, b}\left\langle\check{\Sigma}_{w} \mid u\right\rangle$. In other words, $\check{\Sigma}_{b w}=b \check{\Sigma}_{w}$.

Corollary 16. 1. For $w \in Y^{+}$, the polynomial $\check{\Sigma}_{w}$ is proper and homogenous of degree $|w|$, for $\operatorname{deg}\left(y_{i}\right)=i$, and of rational positive coefficients. 
2.

$$
\sum_{w \in Y^{*}} w \otimes w=\sum_{w \in Y^{*}} \check{\Sigma}_{w} \otimes \Sigma_{w}=\prod_{l \in \mathcal{L} y n Y}^{\searrow} \exp \left(\check{\Sigma}_{l} \otimes \Sigma_{l}\right)
$$

3. The family $\mathcal{L} y n Y$ forms a transcendence basis ${ }^{30}$ of the quasi-shuffle algebra and the family of proper polynomials of rational positive coefficients defined by, for any $w=l_{1}^{i_{1}} \ldots l_{k}^{i_{k}}$ with $l_{1}>\ldots>l_{k}$ and $l_{1}, \ldots, l_{k} \in \mathcal{L} y n Y$,

$$
\chi_{w}=\frac{l_{1}^{\mathbf{L} i_{1}}+\ldots+\ldots l_{k}^{\mathbf{L} i_{k}}}{i_{1} ! \ldots i_{k} !}
$$

forms a basis of the quasi-shuffle algebra.

4. Let $\left\{\xi_{w}\right\}_{w \in Y^{*}}$ be the basis of the envelopping algebra $\mathcal{U}\left(\mathcal{L}_{i} e_{\mathbb{Q}}\langle X\rangle\right)$ obtained by duality with the basis $\left\{\chi_{w}\right\}_{w \in Y^{*}}$ :

$$
\forall u, v \in Y^{*}, \quad\left\langle\chi_{v} \mid \xi_{u}\right\rangle=\delta_{u, v} .
$$

Then the family $\left\{\xi_{l}\right\}_{l \in \mathcal{L} y n Y}$ forms a basis of the free Lie algebra $\mathcal{L}_{i} e_{\mathbb{Q}}\langle Y\rangle$.

Proof. 1. The proof can be done by induction on the length of $w$ using the fact that the product \pm conserve the property, l'homogenity and rational positivity of the coefficients.

2. Expressing $w$ in the basis $\left\{\check{\Sigma}_{w}\right\}_{w \in Y^{*}}$ of the quasi-shuffle algebra and then in the basis $\left\{\Sigma_{w}\right\}_{w \in Y^{*}}$ of the envelopping algebra, we obtain successively

$$
\begin{aligned}
\sum_{w \in Y^{*}} w \otimes w & =\sum_{w \in Y^{*}}\left(\sum_{u \in X^{*}}\left\langle\Sigma_{u} \mid w\right\rangle \check{\Sigma}_{u}\right) \otimes w \\
& =\sum_{u \in Y^{*}} \check{\Sigma}_{u} \otimes\left(\sum_{w \in X^{*}}\left\langle\Sigma_{u} \mid w\right\rangle w\right) \\
& =\sum_{u \in Y^{*}}^{\check{\Sigma}_{u} \otimes \Sigma_{u}} \\
& =\sum_{\substack{l_{1}>\ldots>l_{k} \\
i_{1}, \ldots, i_{k} \geq 1}} \frac{\check{\Sigma}_{l_{1}}^{\omega i_{1}}+\ldots \uplus \check{\Sigma}_{l_{k}} i_{k}}{i_{1} ! \ldots i_{k} !} \otimes \Sigma_{l_{1}}^{i_{1}} \ldots \Sigma_{l_{k}}^{i_{k}} \\
& =\prod_{l \in \mathcal{L} y n Y}^{\searrow} \sum_{i \geq 0} \frac{\check{\Sigma}_{l}+1}{i !} \otimes \Sigma_{l}^{i} \\
& =\prod_{l \in \mathcal{L} y n Y}^{\searrow} \exp \left(\check{\Sigma}_{l} \otimes \Sigma_{l}\right) .
\end{aligned}
$$

\footnotetext{
${ }^{30}$ This result is an analogous of a Radford theorem (see [45]). Thus the bases $\mathcal{L} y n Y$ and $\left\{\check{\Sigma}_{l}\right\}_{l \in \mathcal{L} \text { yn } Y}$ belong to the class of Radford bases, i.e. the class of trancensdence bases, of the quasi-shuffle algebra, as well as the bases $\mathcal{L} y n X$ and $\left\{S_{l}\right\}_{l \in \mathcal{L} y n X}$ belong to the class of Radford bases of the shuffle algebra.
} 
3. For $w=l_{1}^{i_{1}} \ldots l_{k}^{i_{k}}$ with $l_{1}, \ldots, l_{k} \in \mathcal{L} y n Y$ and $l_{1}>\ldots>l_{k}$, by Proposition 19, the polynomial of rational positive coefficients $\check{\Sigma}_{w}$ is lower triangular :

$$
\check{\Sigma}_{w}=\frac{\check{\Sigma}_{l_{1}}^{\leftarrow+i_{1}} \leftarrow \ldots+\check{\Sigma}_{l_{k}}^{\mathbf{\omega} i_{k}}}{i_{1} ! \ldots i_{k} !}=w+\sum_{v<w,(v)=(w)} c_{v} v .
$$

In particular, for any $l_{j} \in \mathcal{L} y n Y, \check{\Sigma}_{l_{j}}$ is lower triangular :

$$
\check{\Sigma}_{l_{j}}=l_{j}+\sum_{v<l_{j},(v)=\left(l_{j}\right)} c_{v} v .
$$

Hence, $\check{\Sigma}_{w}=\chi_{w}+\chi_{w}^{\prime}$, where $\chi_{w}^{\prime}$ is a proper polynomial of $\mathbb{Q}\langle Y\rangle$ of rational positive coefficients. We deduce then the support of $\chi_{w}$ contains words which are less than $w$ and $\left\langle\chi_{w} \mid w\right\rangle=1$. Thus, the proper polynomial $\chi_{w}$ of rational positive coefficients is lower triangular :

$$
\begin{aligned}
\chi_{w} & =w+\sum_{v<w,(v)=(w)} c_{v} v, \\
\Rightarrow \quad \forall l \in \mathcal{L} y n Y, \quad \chi_{l} & =l+\sum_{v<l,(v)=(l)} c_{v} v .
\end{aligned}
$$

It follows then expected results.

4. By duality, for $w \in Y^{*}$, the proper polynomial $\xi_{w}$ is upper triangular. In particular, for any $l \in \mathcal{L} y n Y$, the proper polynomial $\xi_{l}$ is upper triangular :

$$
\xi_{l}=l+\sum_{v>l,(v)=(l)} d_{v} v .
$$

Hence, the family $\left\{\xi_{l}\right\}_{l \in \mathcal{L} y n Y}$ is free and its elements verify an analogous of the generalized criterion of Friedrichs :

- for $w \in \mathcal{L} y n Y$, one has $\left\langle\chi_{w} \mid \xi_{l}\right\rangle=\delta_{w, l}$,

- for $w \notin \mathcal{L} y n Y, w=l_{1} \ldots l_{n}$ with $l_{1}, \ldots, l_{n} \in \mathcal{L} y n Y$ and $l_{1}>\ldots>l_{n}$, one has $\left\langle\chi_{w} \mid \xi_{l}\right\rangle=\left\langle\chi_{l_{1}}\right.$ เ $\ldots$ เ $\left.\chi_{l_{n}} \mid \xi_{l}\right\rangle=0$.

Moreover, the polynomials $\xi_{l}$ 's are primitive : by Corollary $16(3)$, one has

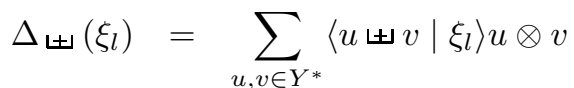

$$
\begin{aligned}
& =\sum_{u \in Y^{+}}\left\langle u \omega 1_{Y^{*}} \mid \xi_{l}\right\rangle u \otimes 1_{Y^{*}}+\sum_{v \in Y^{+}}\left\langle 1_{Y^{*}} \uplus v \mid \xi_{l}\right\rangle 1_{Y^{*}} \otimes v \\
& +\sum_{u, v \in Y^{+}}\left\langle u \amalg v \mid \xi_{l}\right\rangle u \otimes v+\left\langle 1_{Y^{*}} \uplus 1_{Y^{*}} \mid \xi_{l}\right\rangle 1_{Y^{*}} \otimes 1_{Y^{*}} \\
& =\xi_{l} \otimes 1_{Y^{*}}+1_{Y^{*}} \otimes \xi_{l} .
\end{aligned}
$$

Because, after decomposing $u$ and $v$ on the basis $\left\{\chi_{l}\right\}_{l \in \mathcal{L} y n Y}$ and by the previous criterion, the third term is vanishing. The last one is also vanishing since the $\xi_{l}$ 's are proper. By a theorem of Viennot, we obtain then the expected result. 


\subsection{Proof of Theorem 2}

Applying the tensor product of isomorphisms $\mathrm{H} \otimes \mathrm{Id}$ (Proposition 1) on the diagonal series (Corollary 16(ii)), the infinite factorization, by Lyndon words, of the noncommutative generating series of harmonic sums follows ${ }^{31}$ :

$$
\mathrm{H}(N)=\sum_{w \in Y^{*}} \mathrm{H}_{w}(N) w=\prod_{l \in \mathcal{L} y n Y}^{\searrow} \exp \left(\mathrm{H}_{\check{\Sigma}_{l}}(N) \Sigma_{l}\right)
$$

\section{Annexe B : differential realization}

To facilitate reading, the following results are placed in this Annex which can be skipped by readers already familiar with the techniques developed by Fliess (and adapted by us for studies in this paper).

\subsection{Polysystem and convergence criterion}

\subsubsection{Serial estimates from above}

Here, generalizing a little, $\mathbb{K}$ is supposed a $\mathbb{C}$-algebra and a complete normed vector space equipped with a norm denoted by $\|$.$\| .$

For any $n \in \mathbb{N}, X^{\geq n}$ denotes the set of words over $X$ of length greater than or equal to $n$. The set of formal power series (resp. polynomials) on $X$, is denoted by $\mathbb{K}\langle\langle X\rangle\rangle$ (resp. $\mathbb{K}\langle X\rangle$ ).

Definition 17 ([25, 39]). Let $\xi, \chi$ be real positive functions over $X^{*}$. Let $S \in$ $\mathbb{K}\langle\langle X\rangle\rangle$.

1. $S$ will be said $\xi$-exponentially bounded from above if it verifies

$$
\exists K \in \mathbb{R}_{+}, \exists n \in \mathbb{N}, \forall w \in X^{\geq n}, \quad\|\langle S \mid w\rangle\| \leq K \xi(w) /|w| !
$$

We denote by $\mathbb{K}^{\xi-\mathrm{em}}\langle\langle X\rangle\rangle$ the set of formal power series in $\mathbb{K}\langle\langle X\rangle\rangle$ which are $\xi$-exponentially bounded from above.

2. $S$ verifies the $\chi$-growth condition if it satisfies

$$
\exists K \in \mathbb{R}_{+}, \exists n \in \mathbb{N}, \forall w \in X^{\geq n}, \quad\|\langle S \mid w\rangle\| \leq K \chi(w)|w| ! .
$$

We denote by $\mathbb{K}^{\chi-\mathrm{gc}}\langle\langle X\rangle\rangle$ the set of formal power series in $\mathbb{K}\langle\langle X\rangle\rangle$ verifying the $\chi$-growth condition.

\footnotetext{
${ }^{31}$ This proof omitted in previous versions uses mainly the results presented in this annexe that have not been published earlier but have already been presented at various workshops. It is an analogous way to obtain the infinite factorization, by Lyndon words over the alphabet $X$, of the noncommutative generating series of polylogarithms (see Theorem 3) by applying the tensor product of isomorphisms Li $\otimes$ Id (see Proposition 1) on the diagonal series, over $X$.
} 
Lemma 24. We have

$$
R=\sum_{w \in X^{*}}|w| ! w \Rightarrow\left\langle R^{\uplus 2} \mid w\right\rangle=\sum_{\substack{u, v \in X^{*} \\ \operatorname{supp}(u \amalg v) \ni w}}|u| !|v| ! \leq 2^{|w|}|w| ! .
$$

Proof. One has

$$
\begin{aligned}
\sum_{\substack{u, v \in X^{*} \\
\operatorname{supp}(u \amalg v) \ni w}}|u| !|v| ! & =\sum_{k=0} \sum_{\substack{k|=k, k|=|w|-k \\
\operatorname{supp}(u \amalg v) \ni w}} k !(|w|-k) ! \\
& =\sum_{k=0}^{k w \mid}\left(\begin{array}{c}
|w| \\
k
\end{array}\right) k !(|w|-k) ! \\
& =\sum_{k=0}^{k w \mid}|w| ! \\
& =(1+|w|)|w| ! .
\end{aligned}
$$

By induction on the length of $w$, one has $1+|w| \leq 2^{|w|}$. It follows the expected result.

Proposition 20. Let $S_{1}$ and $S_{2}$ verifying the growth condition. Then $S_{1}+S_{2}$ and $S_{1} ш S_{2}$ also verifies the growth condition.

Proof. The proof for $S_{1}+S_{2}$ is immediate.

Next, since $\left\|\left\langle S_{i} \mid w\right\rangle\right\| \leq K_{i} \chi_{i}(w)|w|$ !, for $i=1$ or 2 and for $w \in X^{*}$, then ${ }^{32}$

$$
\begin{aligned}
\left\langle S_{1} w S_{2} \mid w\right\rangle & =\sum_{\operatorname{supp}(u \amalg v) \ni w}\left\langle S_{1} \mid u\right\rangle\left\langle S_{2} \mid v\right\rangle, \\
\Rightarrow\left\|\left\langle S_{1} w S_{2} \mid w\right\rangle\right\| & \leq K_{1} K_{2} \sum_{\substack{u, v \in X^{*} \\
\operatorname{supp}(u \uplus v) \ni w}}\left(\chi_{1}(u)|u| !\right)\left(\chi_{2}(v)|v| !\right) .
\end{aligned}
$$

Let $K=K_{1} K_{2}$ and let $\chi$ be a real positive function over $X^{*}$ such that

$\forall w \in X^{*}, \quad \chi(w)=\max \left\{\chi_{1}(u) \chi_{2}(v) \mid u, v \in X^{*}\right.$ and $\left.\operatorname{supp}(u w v) \ni w\right\}$.

With the notations in Lemma 24, we get

$$
\left\|\left\langle S_{1} w S_{2} \mid w\right\rangle\right\| \leq K \chi(w)\left\langle R^{\varpi 2} \mid w\right\rangle .
$$

Hence, $S_{1} w S_{2}$ verifies the $\chi^{\prime}$-growth condition with $\chi^{\prime}$ defined as $\chi^{\prime}(w)=$ $2^{|w|} \chi(w)$.

Definition 18 ([25, 39]). Let $\xi$ be a real positive function defined over $X^{*}, S$ will be said $\xi$-exponentially continuous if it is continuous over $\mathbb{K}^{\xi-\mathrm{em}}\langle\langle X\rangle\rangle$. The set of formal power series which are $\xi$-exponentially continuous is denoted by $\mathbb{K}^{\xi-e c}\langle\langle X\rangle\rangle$.

\footnotetext{
${ }^{32}\left\langle S_{1} \sqcup S_{2} \mid w\right\rangle$ is the coefficient of the word $w$ in the power series $S_{1} \sqcup S_{2}$.
} 
Lemma 25 ([25, 39]). For any real positive function $\xi$ defined over $X^{*}$, we have $\mathbb{K}\langle X\rangle \subset \mathbb{K}^{\xi-e c}\langle\langle X\rangle\rangle$. Otherwise, for $\xi=0$, we get $\mathbb{K}\langle X\rangle=\mathbb{K}^{0-e c}\langle\langle X\rangle$. Hence, any polynomial is $0-$ exponentially continuous.

Proposition $21([25,39])$. Let $\xi, \chi$ be a real positive functions over $X^{*}$ and let $P \in \mathbb{K}\langle X\rangle$.

1. Let $S \in \mathbb{K}^{\xi-\mathrm{em}}\left\langle\langle X\rangle\right.$. The right residual of $S$ by $P$ belongs to $\mathbb{K}^{\xi-\mathrm{em}}\langle\langle X\rangle$.

2. Let $R \in \mathbb{K}^{\chi-\mathrm{gc}}\left\langle\langle X\rangle\right.$. The concatenation $S R$ belongs to $\mathbb{K}^{\chi-\mathrm{gc}}\langle\langle X\rangle\rangle$.

Proof. $\quad 1$. Since $S \in \mathbb{K}^{\xi-\mathrm{em}}\langle\langle X\rangle\rangle$ then

$$
\exists K \in \mathbb{R}_{+}, \exists n \in \mathbb{N}, \forall w \in X^{\geq n}, \quad\|\langle S \mid w\rangle\| \leq K \xi(w) /|w| ! .
$$

If $u \in \operatorname{supp}(P):=\left\{w \in X^{*} \mid\langle P \mid w\rangle \neq 0\right\}$ then, for any $w \in X^{*}$, one has $\langle S \triangleright u \mid w\rangle=\langle S \mid u w\rangle$ and $S \triangleright u$ belongs to $\mathbb{K}^{\xi-\mathrm{em}}\langle\langle X\rangle$ :

$$
\exists K \in \mathbb{R}_{+}, \exists n \in \mathbb{N}, \forall w \in X^{\geq n}, \quad\|\langle S \triangleright u \mid w\rangle\| \leq[K \xi(u)] \xi(w) /|w| ! .
$$

It follows then $S \triangleright P$ is $\mathbb{K}^{\xi-\mathrm{em}}\left\langle\langle X\rangle\right.$ by taking $K_{1}=K \max _{u \in \operatorname{supp}(P)} \xi(u)$.

2. Since $R \in \mathbb{K}^{\chi-\mathrm{gc}}\langle\langle X\rangle\rangle$ then

$$
\exists K \in \mathbb{R}_{+}, \exists n \in \mathbb{N}, \forall w \in X^{\geq n}, \quad\|\langle S \mid w\rangle\| \leq K \chi(w)|w| ! .
$$

Let $v \in \operatorname{supp}(P)$ such that $v \neq \epsilon$. Since, for any $w \in X^{*}, R v$ belongs to $\mathbb{K}^{\chi-\mathrm{gc}}\langle\langle X\rangle\rangle$ and one has $\langle R v \mid w\rangle=\langle R \mid v \triangleleft w\rangle$ :

$$
\begin{aligned}
\exists K \in \mathbb{R}_{+}, \exists n \in \mathbb{N}, \forall w \in X^{\geq n}, \quad\|\langle R \mid v \triangleleft w\rangle\| & \leq K \chi(v \triangleleft w)(|w|-|v|) ! \\
& \leq K|w| \chi(w) / \chi(v) .
\end{aligned}
$$

Note that if $v \triangleleft w=0$ then $\langle R v \mid w\rangle=0$ and the previous conclusion holds. It follows then $R P$ is $\mathbb{K}^{\chi-\mathrm{gc}}\langle\langle X\rangle\rangle$ by taking $K_{2}=K \min _{v \in \operatorname{supp}(P)} \chi(v)^{-1}$.

Proposition $22([25,39])$. Two real positive morphisms over $X^{*}, \xi$ and $\chi$ are assumed to verify the condition

$$
\sum_{x \in X} \chi(x) \xi(x)<1
$$

Then for any $F \in \mathbb{K}^{\chi-\mathrm{gc}}\left\langle\langle X\rangle, F\right.$ is continuous over $\mathbb{K}^{\xi-\mathrm{em}}\langle\langle X\rangle$.

Proof. If $\xi, \chi$ verify the upper bound condition then the following power series

$$
\sum_{w \in X^{*}} \chi(w) \xi(w)=\left(\sum_{x \in X} \chi(x) \xi(x)\right)^{*}
$$


is well defined. If $F \in \mathbb{K}^{\chi-\mathrm{gc}}\langle\langle X\rangle\rangle$ and $C \in \mathbb{K}^{\xi-\mathrm{em}}\langle\langle X\rangle\rangle$ then there exists $K_{i} \in \mathbb{R}_{+}$and $n_{i} \in \mathbb{N}$ such that for any $w \in X^{\geq n_{i}}, i=1,2$, one has

$$
\|\langle F \mid w\rangle\| \leq K_{1} \chi(w)|w| ! \quad \text { and } \quad\|\langle C \mid w\rangle\| \leq K_{2} \xi(w) /|w| ! .
$$

Hence,

$$
\begin{gathered}
\forall w \in X^{*},|w| \geq \max \left\{n_{1}, n_{2}\right\}, \quad\|\langle F \mid w\rangle\langle C \mid w\rangle\| \leq K_{1} K_{2} \chi(w) \xi(w), \\
\Rightarrow \quad \sum_{w \in X^{*}}\|\langle F \mid w\rangle\langle C \mid w\rangle\| \leq K_{1} K_{2} \sum_{w \in X^{*}} \chi(w) \xi(w)=K_{1} K_{2}\left(\sum_{x \in X} \chi(x) \xi(x)\right)^{*} .
\end{gathered}
$$

\subsubsection{Upper bounds à la Cauchy}

Let $q_{1}, \ldots, q_{n}$ be commutative indeterminates over $\mathbb{C}$. The algebra of formal power series (resp. polynomials) over $\left\{q_{1}, \ldots, q_{n}\right\}$ with coefficients in $\mathbb{C}$ is denoted by $\mathbb{C} \llbracket q_{1}, \ldots, q_{n} \rrbracket$ (resp. $\left.\mathbb{C}\left[q_{1}, \ldots, q_{n}\right]\right)$.

Definition $19([25,39])$. Let

$$
f=\sum_{i_{1}, \ldots, i_{n} \geq 0} f_{i_{1}, \ldots, i_{n}} q_{1}^{i_{1}} \ldots q_{n}^{i_{n}} \in \mathbb{C} \llbracket q_{1}, \ldots, q_{n} \rrbracket .
$$

We set

$$
\begin{aligned}
E(f) & :=\left\{\rho \in \mathbb{R}_{+}^{n}: \exists C_{f} \in \mathbb{R}_{+} \text {s.t. } \forall i_{1}, \ldots, i_{n} \geq 0,\left|f_{i_{1}, \ldots, i_{n}}\right| \rho_{1}^{i_{1}} \ldots \rho_{n}^{i_{n}} \leq C_{f}\right\} \\
\breve{E}(f) & : \text { interior of } E(f) \text { in } \mathbb{R}^{n} . \\
\mathrm{CV}(f) & :=\left\{q \in \mathbb{C}^{n}:\left(\left|q_{1}\right|, \ldots,\left|q_{n}\right|\right) \in \breve{E}(f)\right\} \quad: \quad \text { convergence domain of } f .
\end{aligned}
$$

The power series $f$ is to be said convergent if $\mathrm{CV}(f) \neq \emptyset$. Let $\mathcal{U}$ be an open domain in $\mathbb{C}^{n}$ and let $q \in \mathbb{C}^{n}$. The power series $f$ is to be said convergent on $q$ (resp. over $\mathcal{U})$ if $q \in \mathrm{CV}(f)$ (resp. $\mathcal{U} \subset \mathrm{CV}(f)$ ). We set

$$
\mathbb{C}^{\mathrm{cv}} \llbracket q_{1}, \ldots, q_{n} \rrbracket=\left\{f \in \mathbb{C} \llbracket q_{1}, \ldots, q_{n} \rrbracket: \mathrm{CV}(f) \neq \emptyset\right\} .
$$

Let $q \in \mathrm{CV}(f)$. There exists the constants $C_{f}, \rho$ and $\bar{\rho}$ such that $\left|q_{1}\right|<\bar{\rho}<$ $\rho, \ldots,\left|q_{n}\right|<\bar{\rho}<\rho$ and, for $i_{1} \ldots, i_{n} \geq 0,\left|f_{i_{1}, \ldots, i_{n}}\right| \rho_{1}^{i_{1}} \ldots \rho_{n}^{i_{n}} \leq C_{f}$. The convergence modulus of $f$ at $q$ is $\left(C_{f}, \rho, \bar{\rho}\right)$.

Suppose that $\mathrm{CV}(f) \neq \emptyset$ and let $q \in \mathrm{CV}(f)$. If $\left(C_{f}, \rho, \bar{\rho}\right)$ is a convergence modulus of $f$ at $q$ then $\left|f_{i_{1}, \ldots, i_{n}} q_{1}^{i_{1}} \ldots q_{n}^{i_{n}}\right| \leq C_{f}\left(\bar{\rho}_{1} / \rho_{1}\right)^{i_{1}} \ldots\left(\bar{\rho}_{1} / \rho_{1}\right)^{i_{n}}$. Hence, at $q$, the power series $f$ is majored termwise by

$$
C_{f} \prod_{k=0}^{m}\left(1-\frac{\bar{\rho}_{k}}{\rho_{k}}\right)^{-1} \text {. }
$$


Hence, $f$ is uniformly absolutely convergent in $\left\{q \in \mathbb{C}^{n}:\left|q_{1}\right|<\bar{\rho}, \ldots,\left|q_{n}\right|<\bar{\rho}\right\}$ which is an open domain in $\mathbb{C}^{n}$. Thus, $\operatorname{CV}(f)$ is an open domain in $\mathbb{C}^{n}$. Since the partial derivation of order $j_{1}, \ldots, j_{n} \geq 0$ of $f$ is estimated by

$$
\left\|D_{1}^{j_{1}} \ldots D_{n}^{j_{n}} f\right\| \leq C_{f} \frac{\partial^{j_{1}+\ldots+j_{n}}}{\partial \bar{\rho}^{j_{1}+\ldots+j_{n}}} \prod_{k=0}^{m}\left(1-\frac{\bar{\rho}_{k}}{\rho_{k}}\right)^{-1} .
$$

Proposition $23([25])$. We have $\mathrm{CV}(f) \subset \mathrm{CV}\left(D_{1}^{j_{1}} \ldots D_{n}^{j_{n}} f\right)$.

Let $f \in \mathbb{C}^{\mathrm{cv}} \llbracket q_{1}, \ldots, q_{n} \rrbracket$ and let $\left\{A_{i}\right\}_{i=0,1}$ be a polysystem defined as follows

$$
A_{i}(q)=\sum_{j=1}^{n} A_{i}^{j}(q) \frac{\partial}{\partial q_{j}}, \quad \text { with } \quad A_{i}^{j}(q) \in \mathbb{C}^{\mathrm{cv}} \llbracket q_{1}, \ldots, q_{n} \rrbracket, j=1, \ldots, n .
$$

Lemma $26([20])$. For $i=0,1$ and $j=1, . ., n$, one has $A_{i} \circ q_{j}=A_{i}^{j}(q)$. Thus,

$$
\forall i=0,1, \quad A_{i}(q)=\sum_{j=1}^{n}\left(A_{i} \circ q_{j}\right) \frac{\partial}{\partial q_{j}} .
$$

Let $\left(\rho, \bar{\rho}, C_{f}\right),\left\{\left(\rho, \bar{\rho}, C_{i}\right)\right\}_{i=0,1}$ be respectively the convergence modulus at

$$
q \in \mathrm{CV}(f) \bigcap_{\substack{i=0,1 \\ j=1, \ldots, n}} \mathrm{CV}\left(A_{i}^{j}\right)
$$

of $f$ and $\left\{A_{i}^{j}\right\}_{j=1, . ., n}$. Let us consider the following monoid morphisms

$$
\begin{aligned}
& \mathcal{A}(\epsilon)=\text { identity } \quad \text { and } \quad C(\epsilon)=1 \text {, } \\
& \forall w=v x_{i}, x_{i} \in X, v \in X^{*}, \quad \mathcal{A}(w)=\mathcal{A}(v) A_{i} \quad \text { and } \quad C(w)=C(v) C_{i}
\end{aligned}
$$

Lemma $27([19])$. For any word $w, \mathcal{A}(w)$ is continuous over $\mathbb{C}^{\mathrm{cv}} \llbracket q_{1}, \ldots, q_{n} \rrbracket$ and, for any $f, g \in \mathbb{C}^{\mathrm{cv}} \llbracket q_{1}, \ldots, q_{n} \rrbracket$, one has

$$
\mathcal{A}(w) \circ(f g)=\sum_{u, v \in X^{*}}\langle u w v \mid w\rangle(\mathcal{A}(u) \circ f)(\mathcal{A}(v) \circ g) .
$$

These notations are extended, by linearity, to $\mathbb{K}\langle X\rangle$ and we will denote $\mathcal{A}(w) \circ f_{\mid q}$ the evaluation of $\mathcal{A}(w) \circ f$ at $q$.

Definition 20 ([19]). Let $f \in \mathbb{C}^{\mathrm{cv}} \llbracket q_{1}, \ldots, q_{n} \rrbracket$. The generating series of the polysystem $\left\{A_{i}\right\}_{i=0,1}$ and of the observation $f$ is given by

$$
\begin{aligned}
\sigma f & \left.:=\sum_{w \in X^{*}} \mathcal{A}(w) \circ f w \in \mathbb{C}^{\mathrm{cv}} \llbracket q_{1}, \ldots, q_{n} \rrbracket\langle X\rangle\right\rangle . \\
\sigma f_{\left.\right|_{q}} & :=\sum_{w \in X^{*}} \mathcal{A}(w) \circ f_{\mid q} w \in \mathbb{C}\langle\langle X\rangle .
\end{aligned}
$$

The last generating series is called Fliess generating series of the polysystem $\left\{A_{i}\right\}_{i=0,1}$ and of the observation $f$ at $q$. 
Lemma 28 ([19]). Let $\left\{A_{i}\right\}_{i=0,1}$ be a polysystem. Then, the map

$$
\sigma:\left(\mathbb{C}^{\mathrm{cv}} \llbracket q_{1}, \ldots, q_{n} \rrbracket, .\right) \quad \longrightarrow \quad\left(\mathbb{C}^{\mathrm{cv}} \llbracket q_{1}, \ldots, q_{n} \rrbracket\langle\langle X\rangle\rangle, \omega\right),
$$

is an algebra morphism, i.e. for any $f, g \in \mathbb{C}^{\mathrm{cv}} \llbracket q_{1}, \ldots, q_{n} \rrbracket$ and $\mu, \nu \in \mathbb{C}$, one has $\sigma(\nu f+\mu h)=\nu \sigma f+\mu \sigma g$ and $\sigma(f g)=\sigma f \sqcup \sigma g$.

Lemma $29([20])$. Let $\left\{A_{i}\right\}_{i=0,1}$ be a polysystem and $f \in \mathbb{C}^{\mathrm{cv}} \llbracket q_{1}, \ldots, q_{n} \rrbracket$. Then

$$
\begin{aligned}
\forall x_{i} \in X, \quad \sigma\left(A_{i} \circ f\right)=x_{i} \triangleleft \sigma f & \in & \mathbb{C}^{\mathrm{cv}} \llbracket q_{1}, \ldots, q_{n} \rrbracket\langle\langle X\rangle\rangle \\
\forall w \in X^{*}, \quad \sigma(\mathcal{A}(w) \circ f)=w \triangleleft \sigma f & \in & \mathbb{C}^{\mathrm{cv}} \llbracket q_{1}, \ldots, q_{n} \rrbracket\langle\langle X\rangle\rangle .
\end{aligned}
$$

Lemma 30 ([25]). Let $\tau=\min _{1 \leq k \leq n} \rho_{k}$ and $r=\max _{1 \leq k \leq n} \bar{\rho}_{k} / \rho_{k}$. We have

$$
\begin{aligned}
\|\mathcal{A}(w) \circ f\| & \leq C_{f} \frac{(n+1)}{(1-r)^{n}} \frac{C(w)|w| !}{\left(\begin{array}{c}
n+|w|-1 \\
|w|
\end{array}\right)}\left[\frac{n}{\tau(1-r)^{n+1}}\right]^{|w|} \\
& \leq C_{f} \frac{(n+1)}{(1-r)^{n}} C(w)\left[\frac{n}{\tau(1-r)^{n+1}}\right]^{|w|}|w| ! .
\end{aligned}
$$

Theorem $22([25])$. Let $K=C_{f}(n+1)(1-r)^{-n}$ and let $\chi$ be the real positive function defined over $X^{*}$ by

$$
\forall i=0,1, \quad \chi\left(x_{i}\right)=\frac{C_{i} n}{\tau(1-r)^{(n+1)}} .
$$

Then the generating series $\sigma f$ of the polysystem $\left\{A_{i}\right\}_{i=0,1}$ and of the observation $f$ satisfies the $\chi$-growth condition.

It is the same for the Fliess generating series $\sigma f_{\mid q}$ of the polysystem $\left\{A_{i}\right\}_{i=0,1}$ and of the observation $f$ at $q$.

\subsection{Polysystems and nonlinear differential equation}

\subsubsection{Nonlinear differential equation (with three singularities)}

Let us consider the following singular inputs ${ }^{33}$

$$
u_{0}(z):=z^{-1} \quad \text { and } \quad u_{1}(z):=(1-z)^{-1}
$$

and the following nonlinear dynamical system ${ }^{34}$

$$
\left\{\begin{array}{l}
y(z)=f(q(z)), \\
\dot{q}(z)=A_{0}(q) u_{0}(z)+A_{1}(q) u_{1}(z), \\
q\left(z_{0}\right)=q_{0},
\end{array}\right.
$$

\footnotetext{
${ }^{33}$ These singular inputs are not included in the studies of Fliess motivated, in particular, by the renormalization of $y(z)$ at $+\infty[19,20]$.

${ }^{34}$ Any differential equation with singularities in $\{a, b, c\}$, via homographic transformation $(z-a)(c-b)(z-b)^{-1}(c-a)^{-1}$, can be changed into a differential equation with singularities in $\{0,1,+\infty\}$ (the singularities of homographic transformations belonging to the group $\mathcal{G}$ ).
} 
where, the state $q=\left(q_{1}, \ldots, q_{n}\right)$ belongs to the complex analytic manifold of dimension $n, q_{0}$ is the initial state, the observation $f$ belongs to $\mathbb{C}^{\mathrm{cv}} \llbracket q_{1}, \ldots, q_{n} \rrbracket$ and $\left\{A_{i}\right\}_{i=0,1}$ is the polysystem defined on (114).

Definition 21 ([27]). The following power series is called transport operator of the polysystem $\left\{A_{i}\right\}_{i=0,1}$ and of the observation $f$

$$
\mathcal{T}:=\sum_{w \in X^{*}} \alpha_{z_{0}}^{z}(w) \mathcal{A}(w)
$$

By the factorization of the monoid by Lyndon words, we have [27]

$$
\mathcal{T}=\left(\alpha_{z_{0}}^{z} \otimes \mathcal{A}\right)\left(\sum_{w \in X^{*}} w \otimes w\right)=\prod_{l \in \mathcal{L} y n X} \exp \left[\alpha_{z_{0}}^{z}\left(S_{l}\right) \mathcal{A}\left(\check{S}_{l}\right)\right]
$$

Let us consider again the Chen generating series $S_{z_{0} \rightsquigarrow z}$ given in (51) of the diffferential forms involed in $(D E)$ of Example 1, i.e. $\omega_{0}(z)=u_{0}(z) d z$ and $\omega_{1}(z)=u_{1}(z) d z$, verifying the upper bound conditions given on (56).

\subsubsection{Asymptotic behaviour of the successive differentiation of the output via extended Fliess fundamental formula}

Theorem 23 ([39]). The Fliess fundamental formula can be extended as follows

$$
y(z)=\mathcal{T} \circ f_{\left.\right|_{q_{0}}}=\sum_{w \in X^{*}}\left\langle S_{z_{0} \rightsquigarrow z} \mid w\right\rangle\left\langle\mathcal{A}(w) \circ f_{\left.\right|_{q_{0}}} \mid w\right\rangle=\left\langle\sigma f_{\left.\right|_{q_{0}}} \| S_{z_{0} \rightsquigarrow z}\right\rangle .
$$

By the factorization of the Lie exponential series L, it follows the expansions of the output $y$ of nonlinear dynamical system with singular inputs,

Corollary 17 ([39]).

$$
\begin{aligned}
y(z) & =\sum_{w \in X^{*}} g_{w}(z) \mathcal{A}(w) \circ f_{\mid q_{0}}, \\
& =\sum_{k \geq 0} \sum_{n_{1}, \ldots, n_{k} \geq 0} g_{x_{0}^{n_{1}} x_{1} \ldots x_{0}^{n_{k}} x_{1}}(z) \operatorname{ad}_{A_{0}}^{n_{1}} A_{1} \ldots \operatorname{ad}_{A_{0}}^{n_{k}} A_{1} e^{\log z A_{0}} \circ f_{\mid q_{0}} \\
& =\prod_{l \in \mathcal{L} y n X} \exp \left(g_{S_{l}}(z) \mathcal{A}\left(\check{S}_{l}\right) \circ f_{\mid q_{0}}\right) \\
& =\exp \left(\sum_{w \in X^{*}} g_{w}(z) \mathcal{A}\left(\pi_{1}(w)\right) \circ f_{\left.\right|_{q_{0}}}\right)
\end{aligned}
$$

where, for any word $w$ in $X^{*}, g_{w}$ belongs to the polylogarithm algebra.

Since $S_{z_{0} \rightsquigarrow z}=\mathrm{L}(z) \mathrm{L}\left(z_{0}\right)^{-1}$ and since $\sigma f_{\left.\right|_{q_{0}}}$ and $\mathrm{L}\left(z_{0}\right)^{-1}$ are invariant by $\partial=d / d z$ then $\partial^{l} y(z)=\left\langle\sigma f_{\left.\right|_{0}} \| \partial^{l} S_{z_{0} \rightsquigarrow z}\right\rangle=\left\langle\sigma f_{\left.\right|_{0}} \| \partial^{l} \mathrm{~L}(z) \mathrm{L}\left(z_{0}\right)^{-1}\right\rangle$, for $l \geq 0$.

With the notations of Proposition 3 , we get

$$
\partial^{l} y(z)=\left\langle\sigma f_{\left.\right|_{q_{0}}} \|\left[P_{l}(z) \mathrm{L}(z)\right] \mathrm{L}\left(z_{0}\right)^{-1}\right\rangle=\left\langle\sigma f_{\left.\right|_{q_{0}}} \triangleright P_{l}(z) \| \mathrm{L}(z) \mathrm{L}\left(z_{0}\right)^{-1}\right\rangle .
$$


For $z_{0}=\varepsilon \rightarrow 0^{+}$, the asymptotic behaviour and the renormalization at $z=1$ of $\partial^{l} y(z)$ (or the asymptotic expansion and the renormalization of its Taylor coefficients at $+\infty$ ) are deduced from Proposition 5 and extend a little bit the results of [39] as follows

Corollary 18. For any integer l,we have

$$
\begin{aligned}
\partial^{l} y(1) \underset{\varepsilon \rightarrow 0^{+}}{\simeq} & \left\langle\sigma f_{\left.\right|_{q_{0}}} \triangleright P_{l}(1-\varepsilon) \| e^{-x_{1} \log \varepsilon} Z_{\varpi} e^{-x_{0} \log \varepsilon}\right\rangle \\
& =\sum_{w \in X^{*}}\left\langle\mathcal{A}(w) \circ f_{\left.\right|_{q_{0}}} \mid w\right\rangle\left\langle P_{l}(1-\varepsilon) e^{-x_{1} \log \varepsilon} Z_{\amalg} e^{-x_{0} \log \varepsilon} \mid w\right\rangle .
\end{aligned}
$$

Corollary 19. The differentiation of order $l \in \mathbb{N}$ of the output $y$ of the dynamical system (119) is a $\mathcal{C}$-combination of the elements $g$ belonging to the polylogarithm algebra. If its ordinary Taylor expansion exists then the coefficients of this expansion belong to the algebra of harmonic sums and there exists algorithmically computable coefficients $a_{i} \in \mathbb{Z}, b_{i} \in \mathbb{N}$ and $c_{i}$ belong to the $\mathbb{C}$-algrebra generated by $\mathcal{Z}$ and by the Euler's $\gamma$ constant, such that

$$
\partial^{l} y(z)=\sum_{n \geq 0} y_{n}^{(l)} z^{n}, \quad y_{n}^{(l)} \underset{n \rightarrow \infty}{\widetilde{n}} \sum_{i \geq 0} c_{i} n^{a_{i}} \log ^{b_{i}} n .
$$

\subsection{Differential realization}

\subsubsection{Differential realization}

Definition 22. The Lie rank of a formal power series $S \in \mathbb{K}\langle\langle X\rangle\rangle$ is the dimension of the vector space generated by $\left\{S \triangleright \Pi \mid \Pi \in \mathcal{L} i e_{\mathbb{K}}\langle X\rangle\right\}$, or by $\left\{\Pi \triangleleft S \mid \Pi \in \mathcal{L} i e_{\mathbb{K}}\langle X\rangle\right\}$.

Definition 23. Let $S \in \mathbb{K}\langle\langle X\rangle\rangle$ and let us put

$$
\begin{aligned}
\operatorname{Ann}(S) & :=\left\{\Pi \in \mathcal{L} i e_{\mathbb{K}}\langle X\rangle \mid S \triangleright \Pi=0\right\}, \\
\operatorname{Ann}^{\perp}(S) & :=\{Q \in(\mathbb{K}\langle\langle X\rangle\rangle, \omega) \mid Q \triangleright \operatorname{Ann}(S)=0\} .
\end{aligned}
$$

It is immediate that $\mathrm{Ann}^{\perp}(S) \ni S$ and it follows that (see $[20,46]$ )

Lemma 31. Let $S \in \mathbb{K}\langle\langle X\rangle\rangle$. If $S$ is of finite Lie rank, $d$, then the dimension of $\operatorname{Ann}^{\perp}(S)$ equals $d$.

By Lemma 3, the residuals are derivations for shuffle product. Then,

Lemma 32. Let $S \in \mathbb{K}\langle\langle X\rangle$. Then :

1. For any $Q_{1}$ and $Q_{2} \in \mathrm{Ann}^{\perp}(S)$, one has $Q_{1} ш Q_{2} \in \mathrm{Ann}^{\perp}(S)$.

2. For any $P \in \mathbb{K}\langle X\rangle$ and $Q_{1} \in \operatorname{Ann}^{\perp}(S)$, one has $P \triangleleft Q_{1} \in \operatorname{Ann}^{\perp}(S)$.

Definition 24 ([20]). The formal power series $S \in \mathbb{K}\langle\langle X\rangle$ is differentially produced if there exists 
- an integer $d$,

- a power series $f \in \mathbb{K} \llbracket \bar{q}_{1}, \ldots, \bar{q}_{d} \rrbracket$,

- a homomorphism $\mathcal{A}$ from $X^{*}$ maps to the algebra of differential operators generated by

$\mathcal{A}\left(x_{i}\right)=\sum_{j=1}^{d} A_{i}^{j}\left(\bar{q}_{1}, \ldots, \bar{q}_{d}\right) \frac{\partial}{\partial \bar{q}_{j}}, \quad \mathrm{~A}_{i}^{j}\left(\bar{q}_{1}, \ldots, \bar{q}_{d}\right) \in \mathbb{K} \llbracket \bar{q}_{1}, \ldots, \bar{q}_{d} \rrbracket, j=1, \ldots, d$, such that, for any $w \in X^{*},\langle S \mid w\rangle=\mathcal{A}(w) \circ f_{\left.\right|_{0}}$.

The couple $(\mathcal{A}, f)$ is called differential representation of $S$ of dimension $d$.

Proposition 24 ([46]). Let $S \in \mathbb{K}\langle\langle X\rangle\rangle$. If $S$ is differentially produced then it verifies the growth condition and its Lie rank is finite.

Proof. Let $(\mathcal{A}, f)$ be a differential representation of $S$ of dimension $d$. Then, by the notations of Definition 20, we get

$$
\sigma f_{\left.\right|_{0}}=S=\sum_{w \in X^{*}}(\mathcal{A}(w) \circ f)_{\left.\right|_{0}} w
$$

For any $j=1, . ., d$, we put

$$
\begin{aligned}
T_{j} & =\sum_{w \in X^{*}} \frac{\partial(\mathcal{A}(w) \circ f)}{\partial \bar{q}_{j}} w \\
\Longleftrightarrow \forall w \in X^{*}, \quad\left\langle T_{j} \mid w\right\rangle & =\frac{\partial(\mathcal{A}(w) \circ f)}{\partial \bar{q}_{j}} .
\end{aligned}
$$

Firstly, by Theorem 22, the generating series $\sigma f$ verifies the growth condition. Secondly, for any $\Pi \in \mathcal{L} i e_{\mathbb{K}}\langle X\rangle$ and for any $w \in X^{*}$, one has

$$
\langle\sigma f \triangleright \Pi \mid w\rangle=\langle\sigma f \mid \Pi w\rangle=\mathcal{A}(\Pi w) \circ f=\mathcal{A}(\Pi) \circ(\mathcal{A}(w) \circ f) .
$$

Since $\mathcal{A}(\Pi)$ is a derivation over $\mathbb{K} \llbracket \bar{q}_{1}, \ldots, \bar{q}_{d} \rrbracket$ :

$$
\begin{gathered}
\mathcal{A}(\Pi)=\sum_{j=1}^{d}\left(\mathcal{A}(\Pi) \circ \bar{q}_{j}\right) \frac{\partial}{\partial \bar{q}_{j}}, \\
\Rightarrow \mathcal{A}(\Pi) \circ(\mathcal{A}(w) \circ f)=\sum_{j=1}^{d}\left(\mathcal{A}(\Pi) \circ \bar{q}_{j}\right) \frac{\partial(\mathcal{A}(w) \circ f)}{\partial \bar{q}_{j}}
\end{gathered}
$$

then we deduce that

$$
\begin{gathered}
\forall w \in X^{*}, \quad\langle\sigma f \triangleright \Pi \mid w\rangle=\sum_{j=1}^{d}\left(\mathcal{A}(\Pi) \circ \bar{q}_{j}\right)\left\langle T_{j} \mid w\right\rangle, \\
\Longleftrightarrow \sigma f \triangleright \Pi=\sum_{j=1}^{d}\left(\mathcal{A}(\Pi) \circ \bar{q}_{j}\right) T_{j}
\end{gathered}
$$

That means $\sigma f \triangleright \Pi$ is $\mathbb{K}$-linear combination of $\left\{T_{j}\right\}_{j=1, . ., d}$ and the dimension of the vector space $\operatorname{span}\left\{\sigma f \triangleright \Pi \mid \Pi \in \mathcal{L} i e_{\mathbb{K}}\langle X\rangle\right\}$ is less than or equal to $d$. 


\subsubsection{Fliess' local realization theorem}

Proposition $25([46])$. Let $S \in \mathbb{K}\langle\langle X\rangle\rangle$ such that its Lie rank equals $d$. Then there exists a basis $S_{1}, \ldots, S_{d} \in \mathbb{K}\left\langle\langle X\rangle\right.$ of $\left(\operatorname{Ann}^{\perp}(S), w\right) \cong\left(\mathbb{K} \llbracket S_{1}, \ldots, S_{d} \rrbracket\right.$, w) such that the $S_{i}$ 's are proper and for any $R \in \operatorname{Ann}^{\perp}(S)$, one has

$$
R=\sum_{i_{1}, \ldots, i_{d} \geq 0} \frac{r_{i_{1}, \ldots, i_{n}}}{i_{1} ! \ldots i_{d} !} S_{1}^{\sqcup i_{1}} ш \ldots ш S_{d}^{ш i_{d}},
$$

where the coefficients $\left\{r_{i_{1}, \ldots, i_{d}}\right\}_{i_{1}, \ldots, i_{d} \geq 0}$ belong to $\mathbb{K}$ and $r_{0, \ldots, 0}=\langle R \mid \epsilon\rangle$.

Proof. By Lemma 31, a such basis exists. More precisely, since the Lie rank of $S$ is $d$ then there exists $P_{1}, \ldots, P_{d} \in \mathcal{L} i e_{\mathbb{K}}\langle X\rangle$ such that $S \triangleright P_{1}, \ldots, S \triangleright P_{d} \in$ $(\mathbb{K}\langle\langle X\rangle\rangle, \omega)$ are $\mathbb{K}$-linearly independent. By duality, their exists $S_{1}, \ldots, S_{d} \in$ $(\mathbb{K}\langle\langle X\rangle, \omega)$ such that

$$
\forall i, j=1, . . d, \quad\left\langle S_{i} \mid P_{j}\right\rangle=\delta_{i, j}, \quad \text { and } \quad R=\prod_{i=1}^{d} \exp \left(S_{i} P_{i}\right) .
$$

Expending this product, one obtains, via Poincaré-Birkhoff-Witt theorem, the expected expression for the coefficients $r_{i_{1}, \ldots, i_{d}}=\left\langle R \mid P_{1}^{i_{1}} \ldots P_{d}^{i_{d}}\right\rangle$. Hence, $\left(\mathrm{Ann}^{\perp}(S), \omega\right)$ is generated by $S_{1}, \ldots, S_{d}$.

With the notations of Proposition 25, one has respectively

Corollary 20. If $S \in \mathbb{K}\left[S_{1}, \ldots, S_{d}\right]$ then, for any $i=0,1$ and $j=1, . ., d$, one has $x_{i} \triangleleft S \in \operatorname{Ann}^{\perp}(S)=\mathbb{K}\left[S_{1}, \ldots, S_{d}\right]$.

Corollary 21. The power series $S$ verifies the growth condition if and only if, for any $i=1, . . d, S_{i}$ also verifies the growth condition.

Proof. Assume their exists $j \in[1, . ., d]$ such that $S_{j}$ does not verify the growth condition. Since $S \in \mathrm{Ann}^{\perp}(S)$ then using the decomposition of $S$ on $S_{1}, \ldots, S_{d}$, one obtains a contradiction with the fact that $S$ verifies the growth condition.

Conservely, using Proposition 20, we get the expected results.

Theorem 24 ([20]). The formal power series $S \in \mathbb{K}\langle\langle X\rangle\rangle$ is differentially produced if and only if its Lie rank is finite and if it verifies the $\chi$-growth condition.

Proof. By Proposition 24, one gets a direct proof.

Conversely, since the Lie rank of $S$ equals $d$ then by Proposition 25, by putting $\sigma f_{\left.\right|_{0}}=S$ and, for any $j=1, . ., d, \sigma \bar{q}_{i}=S_{i}$,

1. we choose the observation $f$ as follows

$$
f\left(\bar{q}_{1}, \ldots, \bar{q}_{d}\right)=\sum_{i_{1}, \ldots, i_{d} \geq 0} \frac{r_{i_{1}, \ldots, i_{n}}}{i_{1} ! \ldots i_{d} !} \bar{q}_{1}^{i_{1}} \ldots \bar{q}_{d}^{i_{d}} \in \mathbb{K} \llbracket \bar{q}_{1}, \ldots, \bar{q}_{d} \rrbracket,
$$

such that

$$
\sigma f_{\mid 0}\left(\bar{q}_{1}, \ldots, \bar{q}_{d}\right)=\sum_{i_{1}, \ldots, i_{d} \geq 0} \frac{r_{i_{1}, \ldots, i_{n}}}{i_{1} ! \ldots i_{d} !}\left(\sigma \bar{q}_{1}\right)^{\uplus i_{1}} \omega \ldots ш\left(\sigma \bar{q}_{d}\right)^{\omega i_{d}},
$$


2. it follows that, for $i=0,1$ and for $j=1, . ., d$, the residuals $x_{i} \triangleleft \sigma \bar{q}_{j}$ belongs to $\operatorname{Ann}^{\perp}\left(\sigma f_{\left.\right|_{0}}\right)$ (see also Lemma 32),

3. since $\sigma f$ verifies the $\chi$-growth condition then, by Corollary 21, the generating series $\sigma \bar{q}_{j}$ and $x_{i} \triangleleft \sigma \bar{q}_{j}$ (for $i=0,1$ and for $j=1, . ., d$ ) verify also the growth condition. We then take (see Lemma 29)

$$
\forall i=0,1, \quad \forall j=1, . ., d, \quad \sigma A_{j}^{i}\left(\bar{q}_{1}, \ldots, \bar{q}_{d}\right)=x_{i} \triangleleft \sigma \bar{q}_{j},
$$

by expressing $\sigma A_{j}^{i}$ on the basis $\left\{\sigma \bar{q}_{i}\right\}_{i=1, . ., d}$ of $\operatorname{Ann}^{\perp}\left(\sigma f_{\left.\right|_{0}}\right)$,

4. the homomorphism $\mathcal{A}$ is then determined as follows

$$
\forall i=0,1, \quad \mathcal{A}\left(x_{i}\right)=\sum_{j=0}^{d} A_{j}^{i}\left(\bar{q}_{1}, \ldots, \bar{q}_{d}\right) \frac{\partial}{\partial \bar{q}_{j}},
$$

where, for $i=0,1, j=1, . ., d, A_{j}^{i}\left(\bar{q}_{1}, \ldots, \bar{q}_{d}\right)=\mathcal{A}\left(x_{i}\right) \circ \bar{q}_{j}($ see Lemma 26$)$.

Thus, $(\mathcal{A}, f)$ provides a differential representation ${ }^{35}$ of dimension $d$ of $S$.

Moreover, one also has the following

Theorem 25 ([20]). Let $S \in \mathbb{K}\langle\langle X\rangle\rangle$ supposed to be a differentially produced formal power series. If $(\mathcal{A}, f)$ and $\left(\mathcal{A}^{\prime}, f^{\prime}\right)$ are two differential representations of dimension $n$ of $S$ then there exists a continuous and convergent automorphism $h$ of $\mathbb{K}$ such that, for $w \in X^{*}, g \in \mathbb{K}, h(\mathcal{A}(w) \circ g)=\mathcal{A}^{\prime}(w) \circ(h(g))$ and $f^{\prime}=h(f)$.

Since any rational power series (resp. polynomial), verifies the growth condition and its Lie rank is less or equal to its Hankel rank which is finite [20] then

Corollary 22. Any rational power series and any polynomial over $X$ with coefficients in $\mathbb{K}$ are differentially produced.

Remark 5. 1. By Corollary 20, if $S$ is polynomial then for any $j=1, . ., d$, $S_{j}$ is polynomial. Therefore, for $i=0,1$ and $j=1, . ., d, x_{i} \triangleleft S$ is also polynomial over $X$. In this case, let $(\mathcal{A}, f)$ be a differential representation of $S$ of dimension $d$. Then $f$ and $\left\{A_{j}^{i}\right\}_{j=1, . ., d}^{i=0,1}$ are obviously polynomial on $\bar{q}_{1}, \ldots, \bar{q}_{d}$ and the Lie algebra generated by $\left\{\mathcal{A}\left(x_{i}\right)\right\}^{i=0,1}$ is nilpotent.

2. Note also that, by Theorem 6 , if $S$ is rational over $X$ of linear representation $(\lambda, \mu, \eta)$ then the observation $f\left(q_{1}, \ldots, q_{n}\right)$ equals $\lambda_{1} q_{1}+\ldots+\lambda_{n} q_{n}$ and the polysystem $\{\mathcal{A}(x)\}_{x \in X}$ is obtained by putting

$$
\forall x_{i} \in X, \quad \mathcal{A}\left(x_{i}\right)=\sum_{j=1}^{n}\left(\mu\left(x_{i}\right)\right)_{j}^{i} \frac{\partial}{\partial q_{j}}
$$

yields linear representation not necessarily of minimal dimension [20].

\footnotetext{
${ }^{35}$ In $[20,46]$, the reader can found the discussion on the minimal differential representation.
} 
3. Assume $S \in \mathbb{K} \epsilon \oplus x_{0} \mathbb{K}\langle\langle X\rangle\rangle x_{1}$ and $S$ is a differentially produced. If there exists a basis $S_{1}, \ldots, S_{d}$ of $\left(\mathrm{Ann}^{\perp}(S), \omega\right) \cong\left(x_{0} \mathbb{K}\left\langle\langle X\rangle x_{1}, \omega\right)\right.$ such that

$$
S=\sum_{i_{1}, \ldots, i_{d} \geq 0} r_{i_{1}, \ldots, i_{n}} \frac{S_{1}^{山 i_{1}}}{i_{1} !} ш \ldots ш \frac{S_{d}^{\omega i_{d}}}{i_{d} !} \in\left(\mathbb{K}\left[S_{1}, \ldots, S_{d}\right], ш\right) .
$$

We put $\Sigma_{i}:=\pi_{Y} S_{i}$, for $i=1, . ., d$ and then

$$
\Sigma:=\sum_{i_{1}, \ldots, i_{d} \geq 0} r_{i_{1}, \ldots, i_{n}} \frac{\pi_{1}^{\mathbf{\downarrow} i_{1}}}{i_{1} !} \uplus \ldots \uplus \frac{\Sigma_{d}^{\mathbf{\uplus} i_{d}}}{i_{d} !} \in\left(\mathbb{K}\left[\Sigma_{1}, \ldots, \Sigma_{d}\right], \uplus\right) .
$$

It is a generalization of a Radford's theorem because [29, 30] :

- If $S \in \mathbb{Q}\langle X\rangle$ then (122), (123) are decompositions on Radford bases.

- If $S$ is rational then these are noncommutative partial decompositions. In general one has $\pi_{Y} S \neq \Sigma$ but $\zeta\left(S_{i}\right)=\zeta\left(\Sigma_{i}\right)$ and

$$
\zeta(S)=\zeta(\Sigma)=\sum_{i_{1}, \ldots, i_{d} \geq 0} r_{i_{1}, \ldots, i_{n}} \frac{\zeta\left(S_{1}\right)^{i_{1}}}{i_{1} !} \ldots \frac{\zeta\left(S_{d}\right)^{i_{d}}}{i_{d} !} .
$$

Thus, these yield also identities on polyzêtas at arbitrary weight [37].

\section{References}

[1] E. Abe.- Hopf algebra, Cambridge, 1980.

[2] J. Berstel, and C. Reutenauer.- Rational series and their languages, Springer-Verlag, 1988.

[3] M. Bigotte.- Etude symbolique et algorithmique des fonctions polylogarithmes et des nombres dEuler-Zagier colorés. Thèse, Université Lille, 2000.

[4] L. Boutet de Monvel.- Remark on divergent multizeta series, in Microlocal Analysis and Asymptotic Analysis, RIMS workshop 1397 (2004), pp. 1-9.

[5] N. Bourbaki.- Fonctions of Real Variable, Springer.

[6] N. Bourbaki.- Algebra, chapters II et III, Springer.

[7] P. Cartier.- Développements récents sur les groupes de tresses. Applications à la topologie et à l'algèbre, Sém BOURBAKI, $42^{\text {ème }} 1989-1990, n^{\circ} 716$.

[8] P. Cartier.- Fonctions polylogarithmes, nombres polyzêtas et groupes prounipotents, Sém BOURBAKI, 53 ème 2000-2001, $n^{\circ} 885$.

[9] R. Chari \& A. Pressley.- A guide to quantum group, Cambridge, (1994). 
[10] K.T. Chen.- Iterated path integrals, Bull. Amer. Math. Soc., vol 83, 1977, pp. 831-879.

[11] G. Duchamp \& C. Reutenauer.- Un critère de rationalité provenant de la géométrie noncommutative, Invent. Math., pp. 613-622, (1997).

[12] Costermans, C., Enjalbert, J.Y. and Hoang Ngoc Minh.- Algorithmic and combinatoric aspects of multiple harmonic sums, Discrete Mathematics 8 Theoretical Computer Science Proceedings, 2005.

[13] G. H. E. Duchamp, C. Tollu.-- Sweedler's duals and Schützenberger's calculus, "Conference on Combinatorics and Physics", arXiv: 0712.0125v3.

[14] V. Drinfel'd.- Quantum group, Proc. Int. Cong. Math., Berkeley, 1986.

[15] V. Drinfel'd.- Quasi-Hopf Algebras, Len. Math. J., 1, 1419-1457, 1990.

[16] V. Drinfel'd.- On quasitriangular quasi-hopf algebra and a group closely connected with $\operatorname{gal}(\bar{q} / q)$, Leningrad Math. J., 4, 829-860, 1991.

[17] J. Ecalle.- ARI/GARI, la dimorphie et l'arithmétique des multizêtas : un premier bilan, J. Th. des nombres de Bordeaux, 15, (2003), pp. 411-478.

[18] Fliess M.- Matrices de Hankel, J. M. Purs. Appl., 53, pp. 197-222, (1974).

[19] Fliess M.- Fonctionnelles causales non linéaires et indéterminées non commutatives, Bull. SMF, N¹09, pp. 3-40, (1981).

[20] Fliess M.- Réalisation locale des systèmes non linéaires, algèbres de Lie filtrées transitives et séries génératrices non commutatives, Inventiones Mathematicae, Volume 71, Number 3 / mars 1983, pp. 521-537.

[21] K. Ihara, M. Kaneko \& D. Zagier.- Derivation and double shuffle relations for multiple zetas values, Compositio Math. 142 (2006), pp. 307-338.

[22] T.Q.T. Lê \& J. Murakami.- Kontsevich's integral for Kauffman polynomial, Nagoya Math., pp 39-65, 1996.

[23] C. Malvenuto \& C. Reutenauer.- Duality between quasi-symmetric functions and the solomon descent algebra, J. of Alg. 177 (1995), pp. 967-982.

[24] C. Hespel.-- Une étude des séries formelles noncommutatives pour l'Approximation et l'Identification des systèmes dynamiques, thèse docteur d'état, Université Lille 1, (1998).

[25] Hoang Ngoc Minh.- Contribution au développement d'outils informatiques pour résoudre des problèmes d'automatique non linéaire, Thèse, Lille, 1990.

[26] Hoang Ngoc Minh.- Input/Output behaviour of nonlinear control systems : about exact and approximated computations, IMACS-IFAC Symposium, Lille, Mai 1991. 
[27] Hoang Ngoc Minh, G. Jacob, N. Oussous.- Input/Output Behaviour of Nonlinear Control Systems : Rational Approximations, Nilpotent structural Approximations, in Analysis of controlled Dynamical Systems, (B. Bonnard, B. Bride, J.P. Gauthier \& I. Kupka eds.), Progress in Systems and Control Theory, Birkhäuser, 1991, pp. 253-262.

[28] Hoang Ngoc Minh.- Summations of Polylogarithms via Evaluation Transform, dans Math. \& Computers in Simulations, 1336, pp 707-728, 1996.

[29] Hoang Ngoc Minh.- Fonctions de Dirichlet d'ordre $n$ et de paramètre $t$, dans Discrete Mathematics 180, pp 221-242, 1998.

[30] Hoang Ngoc Minh.- Calcul symbolique non commutatif : aspects combinatoires des fonctions spéciales et des nombres spéciaux, HDR, Lille 2000.

[31] Hoang Ngoc Minh, G. Jacob.- Symbolic integration of meromorphic differential systems via Dirichlet functions, Discrete Mathematics 210 (2000), pp 87-116.

[32] Hoang Ngoc Minh, Jacob G., N.E. Oussous, M. Petitot.- Aspects combinatoires des polylogarithmes et des sommes d'Euler-Zagier, journal électronique du Séminaire Lotharingien de Combinatoire, B43e, (2000).

[33] Hoang Ngoc Minh, Jacob G., N.E. Oussous, M. Petitot.- De l'algèbre des $\zeta$ de Riemann multivariées l'algèbre des $\zeta$ de Hurwitz multivariées, journal électronique du Séminaire Lotharingien de Combinatoire, 44, (2001).

[34] Hoang Ngoc Minh \& M. Petitot.- Lyndon words, polylogarithmic functions and the Riemann $\zeta$ function, Discrete Math., 217, 2000, pp. 273-292.

[35] Hoang Ngoc Minh, M. Petitot and J. Van der Hoeven.- Polylogarithms and Shuffle Algebra, Proceedings of FPSAC'98, 1998.

[36] Hoang Ngoc Minh, Petitot, M., and Van der Hoeven, J..- L'algèbre des polylogarithmes par les séries génératrices, Proceedings of FPSAC'99, 1999.

[37] Hoang Ngoc Minh.- Finite polyzêtas, Poly-Bernoulli numbers, identities of polyzêtas and noncommutative rational power series, Proceedings of $4^{\text {th }}$ International Conference on Words, pp. 232-250, 2003.

[38] Hoang Ngoc Minh.- Differential Galois groups and noncommutative generating series of polylogarithms, in "Automata, Combinatorics and Geometry", 7th World Multi-conference on Systemics, Cybernetics and Informatics, Florida, 2003.

[39] Hoang Ngoc Minh.- Algebraic Combinatoric Aspects of Asymptotic Analysis of Nonlinear Dynamical System with Singular Inputs, Acta Academiae Aboensis, Ser. B, Vol. 67, no. 2, (2007), pp. 117-126.

[40] M. Hoffman.- The Multiple harmonic series, Pacific Journal of Mathematics, 152, 2 (1992), pp. 275-290. 
[41] M. Hoffman.- The algebra of multiple harmonic series, J. of Alg., (1997).

[42] G. Hochschild.- The structure of Lie groups, Holden-Day, (1965).

[43] G. Racinet.- Doubles mélanges des polylogarithmes multiples aux racines de l'unité, Publications Mathématiques de l'IHÉS, 95 (2002), pp. 185-231.

[44] R. Ree.- Lie elements and an algebra associated with shuffles, Ann. of Math, 68 (1958), 210-220.

[45] C. Reutenauer.- Free Lie Algebras, London Math. Soc. Monographs, 1993.

[46] C. Reutenauer.- The local realisation of generating series of finite Lie rank. Algebraic and Geometric Methods In Nonlinear Control Theory. 33-43.

[47] M.P. Schützenberger.- On the definition of a family of automata, Information and Control, 4 (1961) 245-270.

[48] M. Waldschmidt.- Hopf Algebra and Transcendental numbers, "Zetafunctions, Topology and Quantum Physics 2003", Kinki : Japan (2003).

[49] El Wardi.- Mémoire de DEA, Université de Lille, 1999.

[50] D. Zagier.- Values of zeta functions and their applications, in "First European Congress of Mathematics", vol. 2, Birkhäuser (1994), pp. 497-512. 